\title{
ESTUDO DAS VARIABILIDADES ESPECTROSCÓPICAS DA ESTRELA $\eta$ CENTAURI
}

\author{
Ronaldo Savarino Levenhagen
}

Orientador: Prof. Dr. Nelson Vani Leister

Dissertação apresentada ao Instituto Astronômico e Geofísico da Universidade de São Paulo, como requisito parcial para a obtenção do grau de Mestre em Ciências (ÁREA: ASTRONOMIA). 
Aos meus pais Ronaldo e Miriam 


\section{AGRADECIMENTOS}

Ao meu orientador Prof. Dr. Nelson Vani Leister, pela sua orientação, confiança, amizade, paciência e incentivo. Em especial por me ensinar a ensaiar vôos mais altos...

Ao Prof. Dr. Eduardo Janot Pacheco, pelo incentivo, amizade e discussões acadêmicas.

À Dra. A.M. Hubert, pelo auxílio com a fotometria.

Ao colega Christophe Morisset, por me ensinar a trabalhar com o pacote IDL.

À minha namorada Clésia e família, pelo carinho, atenção e paciência nos momentos difíceis.

Aos meus irmãos Ricardo e Renata, pelo apoio e carinho nas situações mais adversas.

Aos bons amigos do IAGUSP: Eduardo Amôres, Iran, Mauro, Roseli, Lucimara, Áurea, Júlio Camargo, Jocel, Jenai, Armando, Marcos Vinícius, Anselmo, Isabel, Alessandro, Paula, Wilton, Juan Leyton, Dimitri, Nelsão, Adriano, Jaqueline, Anderson, Marcelo Emílio, Bruno, Luciana Pompéia, Amelia, Hélio, Jairo, Flávio (Fetza), Pedro, André Escudero, Eduardo Cypriano, Elysandra, Mônica, Antonio Pereyra, Rocío, Marcelo Allen, Dinah, Rodrigo Carrasco, Héctor, Hektor, Gustavo.

Aos bons amigos do IFUSP: Henady, André Queirós, Felipão, Michel, Thomas Haddad, Tomás Lamas, Daniel Franco, Alberto Marcus, César, Benê, Mihara, Alexandre "Comuna", Roberto "Fismático", Rachel, Mayra, Carlinha, Suzana, Camon, Vinícius, Luciana (Atlética), Rubão, Güappo, Valdir (Xerox).

A todos os outros colegas do IAG pelo carinho e amizade.

Aos docentes do Instituto pela minha formação acadêmica.

A todos os funcionários do IAG que tornaram esta dissertação possível de ser realizada, em especial à Marina, Carminha, Conceição, Ana, Marcelo Bazan, Patrícia Regina, Vera (Geofísica), Rosana (Geofísica). Em especial aos meus amigos da portaria Oscar (falecido) e João, devido ao auxílio e atenção prestados nas incontáveis noites que passei no IAG para concluir este trabalho.

O desenvolvimento deste estudo só foi possível com o suporte financeiro da FAPESP (sob contrato 98/00497-0). 
Dans la diversité infinie des phenomènes singuliers, la science ne peut chercher que les invariants. J. Monod ("Le Hasard et la Necessité", Seuil) 


\section{RESUMO}

A espectroscopia de estrelas Be realizada com alta resolução e alta relação sinal/ruído permite investigar variações temporais rápidas nos perfis de linhas de absorção, usualmente atribuídas às pulsações não radiais, entre outros mecanismos. $\mathrm{O}$ fenômeno Be é transitório para esse tipo de estrelas. Com efeito, seus espectros podem apresentar as características de uma estrela B normal, ou então de uma estrela com envelope circunstelar "frio" (presença de linhas finas de emissão e absorção no espectro visível). Essas estrelas se caracterizam por apresentarem altas velocidades de rotação, entretanto insuficientes para explicar as altas taxas de perda de massa, cujos mecanismos permanecem ainda incompreendidos.

Neste trabalho, adotamos o modelo de pulsações não radiais (PNR) para tentar explicar as variações temporais presentes nos perfis de linha centrados em Hel $\lambda 667.8$ nm. Para este fim, foi utilizado o método CLEANEST para efetuar a determinação de periodicidades, após alguns testes de comparação prévios entre o desempenho desse método e o CLEAN. Ambos os métodos possuem grande eficiência em análises temporais, entretanto na média o método CLEANEST se sobressaiu tanto na determinação de freqüências como de amplitudes.

Além disso, foram estudados outros tipos de variabilidades também presentes nesses perfis, como as variabilidades nas intensidades relativas das asas da linha e a variabilidade na posição de um pico central em quase emissão.

Os dados espectroscópicos analisados constituem quatro conjuntos de espectros obtidos nos anos de 1995, 1996, 1997 e 1998 no MCT/LNA. Há ainda um conjunto de dados fotométricos proveniente do satélite HIPPARCOS relativo aos anos de 1990, 1991 e 1992. As análises dos dados espectroscópicos e fotométricos forneceram resultados que concordam com outros trabalhos sobre $\eta$ Centauri. 


\section{ABSTRACT}

The spectroscopy of Be stars carried out with high resolution and signal to noise relation allows to investigate quick temporal variations in line absorption profiles, usually due to non radial pulsations, among other mechanisms. The Be phenomenon is transitory for such stars. Indeed, their spectra may show characteristics of a normal B star, or a star with a "cold" circumstellar envelope (presence of sharp emission and absorption lines in visible spectrum). These stars are characterized by high speed rotation, however insufficient to explain high mass loss rates, whose mechanisms still remains misunderstood.

In this work, we adopted the non-radial pulsation model (NRP) in order to explain the observed temporal variations present in line profiles centered in Hel $\lambda 667.8 \mathrm{~nm}$. For this sake, it was used the CLEANEST method to carry out periodicity determinations, after some previous comparative tests of the performance between this method and $C L E A N$. Both of them possess great efficiency in temporal analysis, however in average the CLEANEST method stood out both in determining frequencies and amplitudes.

Beyond this, were studied also other kinds of variabilities also present in these profiles, like relative intensity variations in the wings of the profile and the variability in position of the central quasi emission peak.

The analyzed spectroscopic data constitute four sets of spectra obtained in 1995, 1996, 1997 and 1998 at the MCT/LNA. There is still one photometric data set coming from HIPPARCOS satellite spanning from 1990 to 1992. The spectroscopic and photometric data analysis have furnished results that agree with other works on $\eta$ Centauri. 


\section{ÍNDICE}

Página

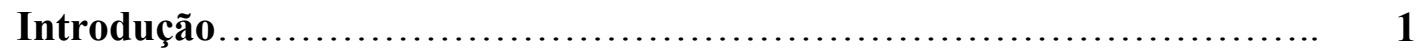

1- As Estrelas Be..................................................... 3

1.1 - Generalidades..................................................... 3

1.2- Observações das Be's ao Longo do Espectro de Radiação............... 6

1.3- O Modelo Rotacional de Struve para o Fenômeno Be.................... 7

1.4- Outros Modelos para o Fenômeno Be.................................. 8

1.5- Variações Rápidas nas Estrelas Be.................................. 9

2- Estudo da Estrela $\eta$ Centauri.................................... 12

2.1- Generalidades................................................... 12

2.2- Instrumentação e Dados Observacionais............................... 13

2.3- Tratamento dos Dados............................................. 18

3- Teoria das Pulsações Não Radiais.................................. 20

3.1- Possíveis Mecanismos de Excitação.................................... 20

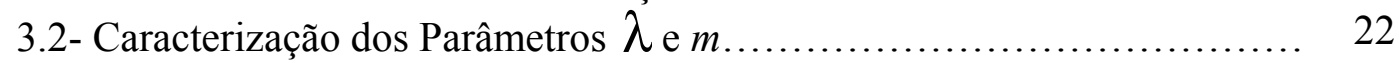

3.3- Equações Básicas das PNR's..................................... 23

3.3.1 - Estrela em equilíbrio estático.................................. 25

3.3.2- Equações das oscilações (análise de perturbação).................. 26

3.3.3- Equações básicas das PNR lineares e não adiabáticas............... 29

3.3.4- Modos Toroidais............................................... 31

3.3.5- Características das PNR adiabáticas............................ 31

3.3.6- Análise local das soluções...................................... 34

4- Métodos de Análise............................................... 37

4.1- Método dos Resíduos............................................. 37

4.2- Método da Transformada de Fourier................................. 39

4.2.1- O Algoritmo CLEAN...................................... 40

4.2.2- O Algoritmo CLEANEST .................................... 41

4.2.3- Álgebra do Algoritmo CLEANEST ............................... 42

4.2.4- Comparação do desempenho dos algoritmos..................... 45

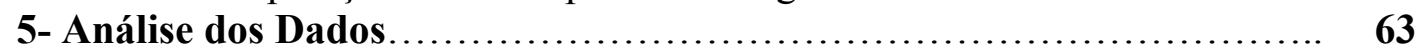

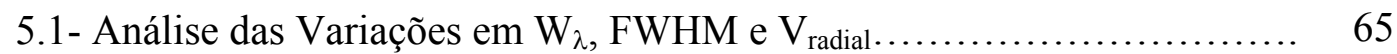

5.2- Aplicação do Método de Telting \& Schrijvers........................ 72

5.3- Análise de Dados de Fotometria.................................... 78

5.4- Análise da Variabilidade Cíclica em V/R............................ 80

5.5- Análise da Variabilidade dos $C Q E$ 's ............................... 83

6- Conclusão e Perspectivas........................................ 87

Referências Bibliográficas......................................... 89 


\section{ÍNDICE DE FIGURAS}

Fig.1. As linhas espectrais de uma estrela com baixa velocidade de rotação (a) aparecem estreitas. Para uma estrela com alta velocidade de rotação, as linhas geradas nos lados opostos da superfície da estrela são desviadas em sentidos opostos, enquanto que o centro da estrela exibe a posição do centro da linha inalterada (b). Como a estrela não pode ser resolvida espacialmente, podemos resultando em um perfil de linha alargado. (adaptado de Böhm-Vitense, 1989)

Fig.2 Esquema morfológico do interior do Sol, mostrando as cavidades de propagação de oscilações com modos $\mathrm{p}$ e g. O aumento da velocidade do som com a profundidaden faz com que haja uma curvatura na trajetória de propagação. $\mathrm{O}$ ponto de reflexão (ou curvatura) é função da freqüência da onda, da velocidade do som e de $\lambda$ (ilustração cedida pelo National Optical Astronomy Observatories - NOAO) .........

Fig.3. Localização no diagrama HR das estrelas indicadas na Tabela1, onde foram detectadas PNR's (adaptado de Unno et al 1989). ...

Fig.4. Representação esquemática do modelo rotacional de Struve (Slettebak, 1988). .. 8

Fig.5a. Modelo de Mancha Fotosférica (adaptado de Vogt, 1983)

Fig.5b. Modelo de PNR, ilustrado para o caso de modo setorial com $\lambda=|\mathrm{m}|=8$ (Vogt, 1983)

Fig.6. Espectros janela (window spectra) dos dados espectroscópicos referentes aos conjuntos de dados de 1996/1997/1998 (Fig.6a), 1997/1998 (Fig.6b) e 1996 (Fig.6c)...

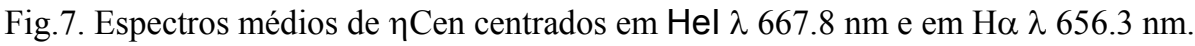

Fig.8. Perfis médios anuais de $\mathrm{H} \alpha \lambda 656.3 \mathrm{~nm}$. Note que o período de atividade da estrela é de cerca de 10 anos (adaptado de Hanuschik et al., 1996).

Fig.9. Padrões modais de oscilações não radiais para 2 modos normais de grau $\lambda=6$ e ordens $m=0$ (esquerda) e $m=3$ (direita) (adaptado de Harvey et al., 1987).

Fig.10. Espectros (à esquerda) e resíduos (à direita) da linha HeI $\lambda 667.8 \mathrm{~nm}$ da estrela $\eta$ Cen. Os espectros estão dispostos em ordem crescente no tempo de cima para baixo, e os resíduos de baixo para cima.

Fig.11. Resíduos dos espectros do dia 09/jun/1998 em escala de cinza................ 38

Fig.12. Gráficos dos pontos aleatórios introduzidos nos dados sintéticos.............. 46

Fig.13. Pontos gerados segundo distribuições de probabilidades distintas (uma para cada ponto).

Fig.14. Sinais igualmente espaçados no tempo e sem ruído (Janela menor).

Fig.15. Sinais igualmente espaçados no tempo e com ruído $\sigma=3.6$.

Fig.16. Sinais desigualmente espaçados no tempo e sem ruído.

Fig.17. Sinais desigualmente espaçados no tempo e com ruído $\sigma=3.6$. 
Fig.18. Sinais igualmente espaçados no tempo, janela maior, sem ruído............. 52

Fig.19. Sinais igualmente espaçados no tempo, janela maior, com ruído $\sigma=23 \ldots \ldots \ldots .53$

Fig.20. Sinais desigualmente espaçados no tempo, com ruído $\sigma=28 \ldots \ldots \ldots \ldots \ldots \ldots . \quad 54$

Fig.21. Sinais igualmente espaçados no tempo, janela maior, com ruído $\sigma=70 \ldots \ldots \ldots .55$

Fig.22. Sinais desigualmente espaçados no tempo, com ruído $\sigma=87 \ldots \ldots \ldots \ldots \ldots \ldots . \quad 56$

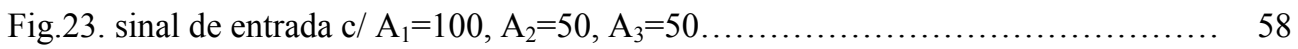

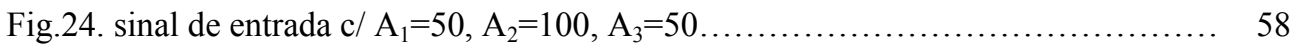

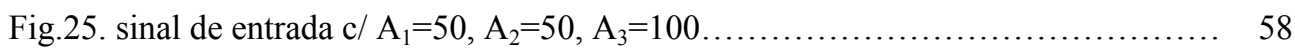

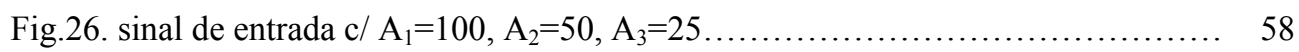

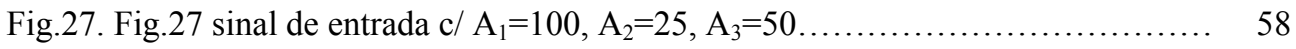

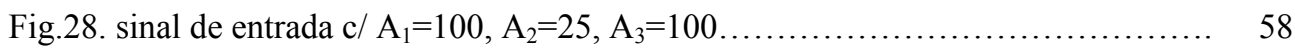

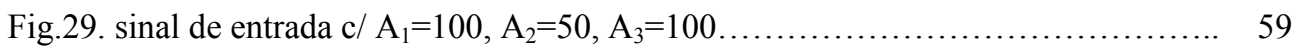

Fig.30. Resultados das análises realizadas com os algoritmos CLEAN e CLEANEST. No histograma à esquerda é mostrado o número de acertos e empates de ambos os algoritmos na determinação das freqüências de entrada, em relação ao número total de freqüências obtidas. No painel à direita, é mostrado o número de acertos e empates na determinação das amplitudes dos sinais.

Fig.31. Periodograma dos resultados do CLEANEST para 1996/1997/1998. Pode-se ver sinais com $0.6 \mathrm{c} / \mathrm{d}, 1.48 \mathrm{c} / \mathrm{d}$ e $5.3 \mathrm{c} / \mathrm{d}$.

Fig.32. Periodograma dos resultados do CLEANEST para os anos 1997/1998

Podem ser observadas as freqüências de 0.6 c/d, 1.48 c/d, 3.8 c/d, 5.3 c/d, 9.2,10.3c/d...

Fig.33. Periodograma dos resultados do CLEANEST para o ano de 1998 mostrando variabilidade em $3.8 \mathrm{c} / \mathrm{d}$.....

Fig.34. Periodograma dos resultados do CLEANEST para o ano de 1997 mostrando as freqüências de $1.48 \mathrm{c} / \mathrm{d}, 3.8 \mathrm{c} / \mathrm{d}, 5.3 \mathrm{c} / \mathrm{d}, 9.2 \mathrm{c} / \mathrm{d}$ e $10.3 \mathrm{c} / \mathrm{d}$.

Fig.35. Periodograma dos resultados do CLEANEST para o ano de 1996 mostrando as freqüências de $0.6 \mathrm{c} / \mathrm{d}, 1.48 \mathrm{c} / \mathrm{d}$ (fraca) e $5.3 \mathrm{c} / \mathrm{d}$.

Fig.36. Gráfico da largura equivalente $\left(\mathrm{W}_{\lambda}\right)$ em função do tempo

(data juliana modificada) dos espectros dos anos de 1996/1997/1998.

Fig.37. Gráfico da largura a meia altura (FWHM) em função do tempo (data juliana modificada) dos espectros dos anos de 1996/1997/1998.

Fig.38. Gráfico da velocidade radial $\left(\mathrm{V}_{\text {radial }}\right)$ em função do tempo (data juliana modificada) dos espectros dos anos de 1996/1997/1998. 
Fig.39. Diagrama em escala de cinza mostrando os resíduos médios dos espectros centrados em $\lambda 667.8 \mathrm{~nm}$. Note a presença de regiões claras dispostas em alternância com regiões mais escuras que se propagam ao longo do perfil de linha. Para maior clareza, os resíduos foram mostrados em fase com a freqüência de 0.6 ciclos/dia e dois ciclos inteiros de oscilação ( 0 a $4 \pi$ radianos).

Fig.40. Diagrama em escala de cinza mostrando os resíduos médios dos espectros centrados em $\lambda 667.8 \mathrm{~nm}$. Os resíduos foram mostrados em fase com a freqüência de $1.48 \mathrm{ciclos} / \mathrm{dia}$.

Fig.41. Diagrama em escala de cinza mostrando os resíduos médios dos espectros centrados em $\lambda 667.8 \mathrm{~nm}$, em fase com a freqüência de 3.8 ciclos $/$ dia.

Fig.42. Diagrama em escala de cinza mostrando os resíduos médios dos espectros centrados em $\lambda 667.8 \mathrm{~nm}$, em fase com a freqüência de 5.3 ciclos/dia.

Fig.43. Diagrama em escala de cinza mostrando os resíduos médios dos espectros centrados em $\lambda 667.8 \mathrm{~nm}$, em fase com a freqüência de 9.2 ciclos $/$ dia.

Fig.44. Diagrama em escala de cinza mostrando os resíduos médios dos espectros centrados em $\lambda 667.8 \mathrm{~nm}$, em fase com a freqüência de 10.3 ciclos/dia....

Fig.45. Amplitudes e fases da freqüência $0.6 \mathrm{c} / \mathrm{d}$ ao longo do perfil de linha do HeI. O aspecto do diagrama de amplitude sugere ser esta freqüência associada a uma oscilação de modo $\mathrm{g}$. A partir do diagrama de fase, podemos estimar $\Delta \phi \sim-\pi$ e o grau da pulsação $\lambda \sim 1$ (Telting, 1997).

Fig.46. Amplitudes e fases da freqüência $1.48 \mathrm{c} / \mathrm{d}$ ao longo do perfil de linha do HeI. O aspecto do diagrama de amplitude sugere ser esta freqüência associada a uma oscilação de modo $\mathrm{g}$. A partir do diagrama de fase, podemos estimar $\Delta \phi \sim--3 \pi$ e o grau da pulsação como sendo $\lambda \sim 3$ (Telting, 1997).

Fig.47. Amplitudes e fases da freqüência $3.8 \mathrm{c} / \mathrm{d}$ ao longo do perfil de linha do HeI. A partir do diagrama de fase, podemos estimar $\Delta \phi \sim-5 \pi$ e o grau da pulsação $\lambda \sim 5$ (Telting, 1997)

Fig.48. Amplitudes e fases da freqüência $5.3 \mathrm{c} / \mathrm{d}$ ao longo do perfil de linha do HeI. A partir dos diagramas de fase, podemos estimar $\Delta \phi \sim-5 \pi$ e o grau da pulsação $\lambda \sim 5$ (Telting, 1997)

Fig.49. Amplitudes e fases da freqüência $9.2 \mathrm{c} / \mathrm{d}$ ao longo do perfil de linha do HeI. A partir do diagrama de fase, podemos estimar $\Delta \phi \sim-5 \pi$ e o grau da pulsação $\lambda \sim 5$ (Telting, 1997)

Fig.50. Amplitudes e fases da freqüência $10.5 \mathrm{c} / \mathrm{d}$ ao longo do perfil de linha do HeI. A partir do diagrama de fase, podemos estimar $\Delta \phi \sim-7 \pi$ o grau da pulsação como sendo $\lambda \sim 7$ (Telting, 1997).

Fig.51. Curva de luz dos dados fotométricos obtidos pelo satélite HIPPARCOS ao longo dos anos de 1990, 1991 e 1992.

Fig.52. Curva de luz dos dados fotométricos obtidos pelo satélite HIPPARCOS, em fase com a freqüência $1.55 \mathrm{c} / \mathrm{d}$, exibindo dois ciclos completos de oscilação ( 0 a $4 \pi$ $\mathrm{rad})$. 
Fig.53. Esquema ilustrativo do mecanismo de variabilidades rápidas nas asas da linha do HeI.

Fig.54. Gráfico da razão V/R relativa aos anos de 1995, 1996, 1997 e 1998.

Fig.55. Modelo fotosférico (Zorec, 1994) a) (linhas cheias) Curvas de iso-velocidade radial para uma estrela vista com $\mathrm{i}=30^{\circ}$, onde $\mathrm{P}$ é o pólo estelar e.$\pm \mathrm{M}$ são os pontos de velocidade radial de valores $\pm \mathrm{V}_{\operatorname{MAX}} \sin$ i. b) (linha cheia) Perfil da linha em Hel $\lambda 447.1 \mathrm{~nm}$ com $V_{\text {MAX }} \sin i=100 \mathrm{~km} \cdot \mathrm{s}^{-1}$. (linha pontilhada) perfil alargado por rotação rígida e mesmo valor de $\mathrm{V} \sin$ i. c) Curvas de iso-velocidade radial com $\mathrm{i}=30^{\circ}$, para o caso de rotação rígida.

Fig.56. Gráfico da posição do $C Q E$ em velocidade ao longo do perfil de linha do He I em função do tempo

Fig.57. Ajuste de senóide com período $\mathrm{p}=60.096$ dias (linha cheia) para as posições dos CQE's (pontos vermelhos) 


\section{ÍNDICE DE TABELAS}

Tab.1. Ocorrência de PNR's em diferentes objetos do diagrama HR (adaptado de Unno et al, 1995)

Tab.2. Principais características físicas da estrela $\eta$ Centauri. Referências:

(1) CDS - Strasbourg; (2) Harmanec, 1988; (3) Underhill, 1982;

(4) Dachs et al., 1988; (5) Hanuschik et al, 1996; (6) Johnson et al, 1966

Tab. 3. Observações espectroscópicas de $\eta$ Cen obtidas no MCT/LNA entre

9/março/95 e 10/junho/98

Tab.4. Alguns exemplos de mecanismos de excitação de oscilações radiais e não radiais, apresentando os principais objetos do diagrama HR associados (Unno, 1989)...

Tab.5. Testes sem ruído com janela de observação menor 48

Tab. 6. Testes com ruído $\sigma=3.6$ e janela de observação menor

Tab. 7. Testes sem ruído em dados desigualmente espaçados no tempo, com a janela de observação correspondente às observações de $\eta$ Cen em 30, 31/05 e 01, 02/06/1996. Tab. 8. Testes com ruído $\sigma=3.6$ e dados desigualmente espaçados no tempo, com a janela de observação correspondente às observações de $\eta$ Cen em 30, 31/05 e 01, $02 / 06 / 1996$

Tab. 9. Testes sem ruído em dados igualmente espaçados no tempo, com janela maior. Neste caso, as freqüências são melhor determinadas do que no caso de janela simples...

Tab. 10. Testes com ruído $\sigma=23$ em dados igualmente espaçados no tempo, com janela maior.

Tab. 11. Testes com ruído $\sigma=28$ em dados desigualmente espaçados no tempo

Tab. 12. Testes com ruído $\sigma=70$ em dados igualmente espaçados no tempo.

Tab. 13. Testes com ruído $\sigma=87$ em dados desigualmente espaçados no tempo

Tab. 14. Testes com os sinais de entrada da Figura 23

Tab. 15. Testes com os sinais de entrada da Figura 24

Tab. 16. Testes com os sinais de entrada da Figura 25.

Tab. 17. Testes com os sinais de entrada da Figura 26.

Tab. 18. Testes com os sinais de entrada da Figura 27

Tab. 19. Testes com os sinais de entrada da Figura 28

Tab. 20. Testes com os sinais de entrada da Figura 29

Tab.21. Resumo das principais freqüências detectadas em velocidade radial, largura equivalente e largura a meia altura através do método CLEANEST ao longo dos anos de 1996,1997 e 1998. 
Tab.22. Resumo das principais freqüências detectadas e as variabilidades observadas. As pesquisas com o CLEANEST para cada série temporal foram feitas buscando 3 freqüências, então o número de graus de liberdade será 3 × $2=6$. Consultando-se uma tabela de valores de Qui-Quadrado, obtemos os valores de significância mostrados......

Tab.23. Resumo dos parâmetros pulsacionais obtidos a partir da análise dos diagramas de amplitude e fase.

Tab.24. Resultados obtidos aplicando o algoritmo CLEANEST às séries temporais de $\mathrm{V} / \mathrm{R}$ relativas aos anos de 1995, 1996, 1997 e 1998

Tab. 25. Resultados da análise temporal dos dados de posição dos $C Q E$ 's referentes aos anos de 1996, 1997 e 1998. O período de maior amplitude é o de 60 dias (0.017 $\mathrm{c} / \mathrm{d})$ 


\section{INTRODUÇÃO}

As estrelas de classe espectral $\mathbf{B e}^{1}$ foram observadas pela primeira vez em 1867 por A.Secchi, o qual descobriu linhas de emissão na estrela $\gamma$ Cas. Desde então, essas estrelas passaram a ser objeto de estudo sobre a origem de numerosas peculiaridades espectrais (Underhill \& Doazan, 1982; Slettebak \& Snow, 1987; Balona et al., 1994; Hanuschik \& Vranken, 1995; Rivinius et al., 1999). Somente a partir da década passada foi possível observar diretamente a geometria dos envelopes circunstelares dessas estrelas através de métodos interferométricos e confirmar a sua estrutura em forma de disco (Stee et al., 1995; Quirrenbach et al., 1997). Até hoje, as questões dos mecanismos físicos que levam à formação do disco circunstelar (Lamers \& Pauldrach, 1991; Bjorkman \& Cassinelli, 1993; Owocki et al., 1996) e da natureza das variabilidades espectroscópicas e fotométricas rápidas (Smith, 1989; Baade \& Balona, 1994) continuam em aberto. Os principais candidatos para explicar as componentes periódicas apresentadas pelas variabilidades rápidas são as Pulsações Não-Radiais (daqui por diante chamadas PNR's) e áreas ativas em corrotação ou manchas estelares. Não obstante, a mais enigmática das variabilidades apresentadas por essas estrelas são os outbursts ${ }^{2}$ das linhas de emissão. Até hoje, nenhuma definição estrita de outbursts foi apresentada, sendo comum caracterizalos por um aumento significativo, em relação à média, da intensidade de certas linhas de emissão Balmer. Normalmente, acredita-se que os outbursts sejam eventos causados pelo aumento da transferência de matéria da estrela para o disco circunstelar. As tentativas de se encontrar um elo entre as variabilidades periódicas rápidas presentes na fotosfera e os episódios de perda de massa nas Be's normalmente não excederam o nível das conjecturas (Smith, 1989). Todavia, Kambe et al., (1993a) e Leister et al. (1994a) encontraram algumas evidências do aumento da amplitude de perfis de linha em momentos próximos da ocorrência de um outburst. Em nenhum caso (excluindo-se algumas estrelas binárias) foi estabelecido um esquema convincente da ocorrência temporal de outbursts, os quais aparentemente se mostram ao acaso.

No cenário do estudo das estrelas Be, $\eta$ Cen em particular é de grande interesse, tendo sido estudada em vários artigos (p.ex. Leister et al., 1994; Balona, 1999; Janot et al., 1999). Ela apresenta diferentes tipos de variabilidades, a saber fotométrica, multifreqüências (possivelmente ligadas às PNR's), variabilidades nas asas de algumas linhas espectrais, bem como a presença de picos de quase emissão no centro de algumas linhas (Rivinius et al., 1999). O propósito científico deste trabalho consiste no estudo das variações, ao longo do tempo, dos perfis de linha fotosféricos centrados no comprimento de onda do Hel $\lambda 667.8 \mathrm{~nm}^{3}$ (singleto formado na transição $2{ }^{1} \mathrm{P}^{0}-3{ }^{1} \mathrm{D}$ do Hélio neutro com nível de energia mais baixo valendo $21.13 \mathrm{eV}$ e nível mais alto valendo $22.97 \mathrm{eV}$ ), de forma a se confirmar a presença de multi-freqüências, o que representa um indício importante da presença de PNR's. Além disso, a ocorrência de variações de intensidade nas asas dessa linha podem estar relacionadas com a atividade da estrela e a passagem de matéria do envelope circunstelar na linha de visão. A presença de picos centrais de quase

\footnotetext{
${ }^{1}$ Os tipos espectrais das estrelas neste trabalho serão exibidos em negrito

${ }^{2}$ Palavras e siglas estrangeiras serão mostradas em itálico

${ }^{3}$ Será adotado neste trabalho o ponto (.) como sinal decimal, e os símbolos de elementos químicos serão exibidos em arial.
} 
emissão pode estar relacionada com efeitos de rotação diferencial da estrela (Zorec, 1988).

Este trabalho está estruturado nos seguintes capítulos: o capítulo 1, que se dedica à caracterização das estrelas Be em geral e suas fenomenologias. O capítulo 2, onde são abordadas as características principais da estrela $\eta$ Centauri, a instrumentação utilizada no estudo espectroscópico e fotométrico, bem como o processo de redução dos dados espectroscópicos. O capítulo 3, onde é feita uma abordagem sobre a teoria das pulsações não radiais (PNR's), seus principais mecanismos de excitação, descrição dos modos de oscilação e uma rápida abordagem sobre a teoria das PNR's adiabáticas e lineares. A seguir, o capítulo 4 trata dos métodos de análise empregados em Sismologia Estelar para a determinação de freqüências relacionadas às PNR's. Nesse contexto, são descritos os métodos CLEAN e CLEANEST de busca de períodos e feita uma comparação do desempenho desses algoritmos através de análises de séries temporais artificiais. $\mathrm{O}$ capítulo 5 apresenta os resultados das análises temporais das variações dos perfis de linha (vpl's) centrados em $\lambda 667.8 \mathrm{~nm}$ e dos dados de fotometria, bem como os resultados das variações em largura equivalente, largura a meia altura e velocidade radial nos espectros. É feita também uma aplicação do método de análise de amplitudes e fases das freqüências descrito em Telting et al.,(1997a,b,c), de onde podem ser inferidos alguns parâmetros pulsacionais das freqüências obtidas anteriormente. Nesse mesmo capítulo é analisada a série temporal da razão $\mathrm{V} / \mathrm{R}$, que representa um indicativo da variação da intensidade das asas da linha com o tempo. Em seguida, é analisada a série temporal da posição, no perfil de linha, dos picos centrais de quase-emissão ( $C Q E$ 's). Finalmente, o capítulo 6 apresenta as conclusões deste trabalho. 


\section{1- As Estrelas Be}

\subsection{Generalidades}

As pulsações estelares, causadas tanto por oscilações radiais quanto não radiais, são observadas em diversas fases da evolução estelar (Gautchy \& Saio,1995), ocupando dessa forma várias regiões do diagrama Hertsprung-Russell (daqui em diante chamado HR). Na década de 70 , as estrelas $\beta$ Cephei eram as únicas variáveis pulsantes quentes conhecidas. Com o surgimento da espectroscopia de alta resolução, passou a ser possível analisar as variabilidades, ao longo do tempo, de diversas linhas fotosféricas (p. ex. $\lambda 667.8 \mathrm{~nm}, \lambda 447.1 \mathrm{~nm}$ ), as quais são alargadas devido à rotação da estrela, conforme ilustrado na Figura 1.

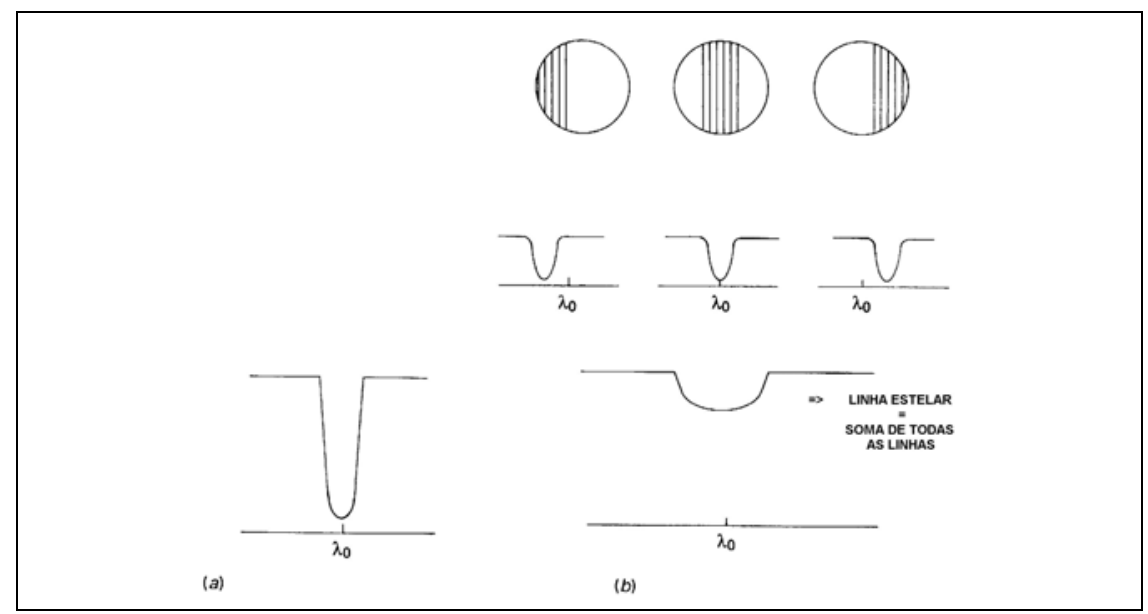

Fig.1. As linhas espectrais de uma estrela com baixa velocidade de rotação (a) aparecem estreitas. Para uma estrela com alta velocidade de rotação, as linhas geradas nos lados opostos da superfície da estrela são desviadas em sentidos opostos, enquanto que o centro da estrela exibe a posição do centro da linha inalterada (b). Como a estrela não pode ser resolvida espacialmente, podemos observar somente a soma dos espectros originados em diferentes partes da superfície estelar, resultando em um perfil de linha alargado. (adaptado de Böhm-Vitense, 1989)

Dessa forma os avanços na espectroscopia, juntamente com o auxílio da fotometria, foram responsáveis pela descoberta de muitas outras estrelas variáveis pulsantes ao longo do diagrama HR conforme mostram a Tabela 1 e Figura 3, com massas entre 4 e $18 \mathrm{M}_{\odot}$ e raios entre 3 e $15 \mathrm{R}_{\odot}$ Essas estrelas, conhecidas de forma genérica como variáveis $\mathbf{O}-\mathbf{B}$, englobam as $\beta$ Cephei, as $S P B$ (slowly pulsating $B$ stars) e as estrelas variáveis $\mathbf{B}$ e Be. No caso das variáveis Be, suas velocidades de rotação variam entre $50 \%$ e $80 \%$ da velocidade de rotação crítica. No diagrama HR, elas se situam na mesma região que as estrelas de tipo $\beta$ Cephei, acima da seqüência principal. 


Tipo de estrela $\quad$ Período $\quad$ Variabilidade $\quad$ Modos de Oscilação

Variáveis Early-type O-B

$\begin{array}{cccc}\text { variáveis } \beta \text { Cephei } & 3 \sim 6 \text { horas } & \text { lum e veloc.radial } & \text { f ou p com } \lambda=2 \sim 4 \\ \text { variáveis } 53 \text { Per } & 0.5 \sim 2 \text { dias } & \text { perfil de linha } & \text { g com } \lambda \text { baixo } \\ \text { variáveis } \zeta \text { Oph } & \sim \text { horas } & \text { perfil de linha } & \text { g e p }\end{array}$

Variáveis A type

$\begin{array}{cccc}\text { estrelas Ap } & 5 \sim 15 \text { min } & \text { luminosidade } & \text { p com baixo } \lambda \\ \text { estrelas } \delta \text { Sct } & 1 \sim 2 \text { horas } & \begin{array}{c}\text { lum. e perfil de } \\ \text { linha }\end{array} & \text { p com baixo } \lambda\end{array}$

\section{Variáveis Anãs Brancas}

\begin{tabular}{|c|c|c|c|}
\hline variáveis DA & $100 \sim 1000 \mathrm{~s}$ & luminosidade & g com baixo $\lambda$ \\
\hline variáveis DB & $100 \sim 1000 \mathrm{~s}$ & luminosidade & g com baixo $\lambda$ \\
\hline variáveis DO & $400 \sim 1600 \mathrm{~s}$ & luminosidade & g com baixo $\lambda$ \\
\hline Sol & $\begin{array}{l}5 \sim 10 \text { min } \\
1 \sim 3 \text { horas }\end{array}$ & $\begin{array}{l}\text { veloc.radial } \\
\text { veloc.radial }\end{array}$ & $\begin{array}{c}\mathrm{p} \operatorname{com} \lambda=0 \sim 1000 \\
\mathrm{~g} \text { com baixo } \lambda\end{array}$ \\
\hline Anãs Late-type & $5 \sim 10 \mathrm{~min}$ & luminosidade & p com baixo $\lambda$ \\
\hline igantes Early-type & $\begin{array}{c}\text { dezenas de } \\
\text { dias }\end{array}$ & $\begin{array}{l}\text { luminosidade e } \\
\text { velocidade radial }\end{array}$ & p e g com baixo $\lambda$ \\
\hline
\end{tabular}

Tab. 1. Ocorrência de PNR's em diferentes objetos do diagrama HR (adaptado de Unno et al., 1995).

Na Tabela 1 acima, os modos de oscilação indicados pela letra g dizem respeito aos chamados modos de gravidade, onde a força restauradora das oscilações é a gravidade. Os modos p são ondas acústicas estacionárias, com a força de restauração sendo dominada pela pressão. Os modos f constituem as oscilações radiais. Ainda na mesma tabela, são mostrados para cada objeto os valores típicos assumidos pelo parâmetro de pulsação $\lambda$ (grau da pulsação), que é um indicativo do número total de planos nodais de oscilação. Um estudo mais pormenorizado sobre os modos de pulsação $\mathrm{p}$ e g será feito mais adiante. Na Figura 2, temos um esquema da propagação de oscilações com modos $\mathrm{p}$ e g no interior do Sol. Em particular, os altos valores de $\lambda$ identificados no Sol, associados às oscilações de 5 minutos, não são identificados em outras estrelas devido à impossibilidade de resolução da superfície das mesmas. 


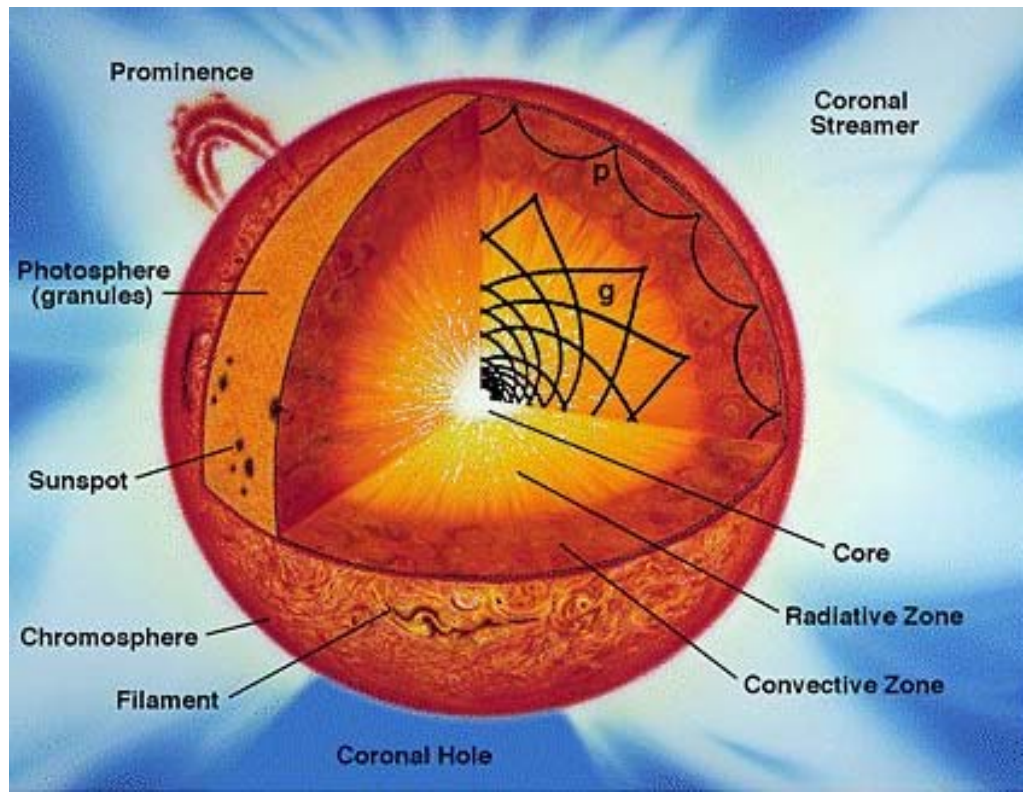

Fig.2 Esquema morfológico do interior do Sol, mostrando as cavidades de propagação de oscilações com modos $\mathrm{p}$ e g. $\mathrm{O}$ aumento da velocidade do som com a profundidade faz com que haja uma curvatura na trajetória de propagação. O ponto de reflexão (ou curvatura) é função da freqüência da onda, da velocidade do som e de $\lambda$. (ilustração cedida pelo National Optical Astronomy Observatories - NOAO)

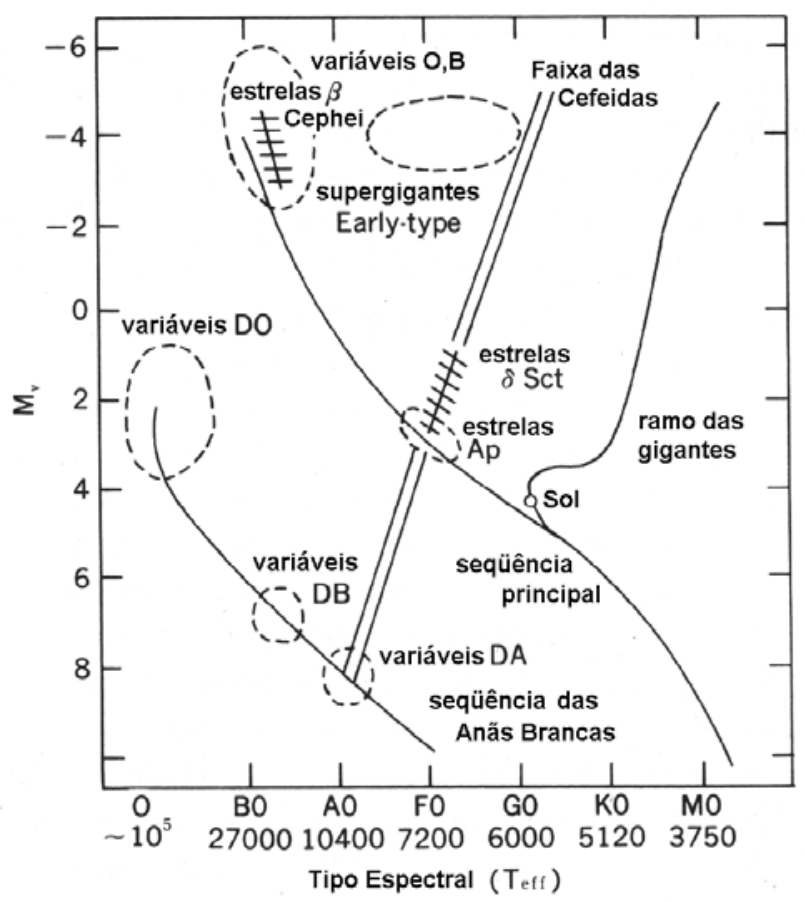

Fig.3. Localização no diagrama HR das estrelas indicadas na Tabela1, onde foram detectadas PNR's (adaptado de Unno et al. 1989).

As estrelas tipo B são estrelas tipicamente azuladas e quentes $(\mathrm{T} \sim 25000 \mathrm{~K})$ que mostram em seu espectro de absorção a presença do He I , do $\mathrm{H}$, e também do $\mathrm{Mg}$ II, Si II e Si III. A teoria clássica das atmosferas estelares explica bem esse espectro de 
absorção, porém existem algumas estrelas tipo B que apresentam linhas de emissão que não são contempladas satisfatoriamente por esta teoria. Para haver distinção entre as estrelas B "normais" e estas que apresentam raias de emissão, acrescenta-se o sufixo e ao tipo espectral destas últimas, sendo chamadas de estrelas Be.

Historicamente, as estrelas Be foram observadas pela primeira vez em 1867 por A.Secchi, o qual descobriu linhas de emissão na estrela $\gamma$ Cas. Os espectros de emissão das Be podem apresentar-se com uma fraca absorção central sobreposta a uma forte emissão (espectro Be) ou então com uma absorção central fina e profunda com ou sem asas em emissão (espectro Be shell). A maioria das observações feitas das Be mostraram que as características das emissões são diferentes de uma estrela para outra, e que mesmo para uma dada estrela os espectros apresentam variações ao longo do tempo. No início dos estudos destes objetos acreditava-se que as estrelas Be e Be shell constituíam duas subclasses diferentes das estrelas B. Entretanto, as observações mostraram que, em escalas de tempo de alguns anos, os espectros de uma dada estrela podem sofrer transições de um espectro Be para um espectro Be shell ou para um espectro B simples, e assim por diante. Por isso acredita-se, hoje, que esses tipos diferentes de espectros constituem fases diferentes e periódicas causadas pela evolução temporal dessas estrelas. Esse comportamento variável das Be's constitui o chamado fenômeno Be.

É útil adotarmos uma definição de estrela $\mathbf{B e}$, no entanto não se pode esquecer que a existência de linhas de emissão provenientes de estrelas não era totalmente desconhecida antes da caracterização do fenômeno Be. Existem estrelas supergigantes que apresentam linhas de emissão, geralmente do $\mathrm{H} \alpha$, que podem ser explicadas pela teoria clássica das atmosferas estelares; também existe uma classe de estrelas conhecidas como Ae-Be de Herbig que são estrelas jovens (pré-seqüência principal) e que devem ser distinguidas das estrelas Be. Acredita-se também que as estrelas Oe e A-shell sejam extensões do fenômeno Be para as temperaturas mais altas e mais baixas respectivamente. Portanto, uma estrela Be é uma estrela não supergigante, do tipo BV a BI (próximas à seqüência principal) que apresenta ou apresentou um espectro de emissão conforme descrito acima. Algumas estrelas Be apresentam linhas de emissão proibidas sendo chamadas de estrelas $\mathbf{B}[\mathbf{e}]$.

\subsection{Observações de Be's ao Longo do Espectro de Radiação}

A principal manifestação do fenômeno Be no visível é a presença de linhas de emissão na série de Balmer (mais intensa no $\mathrm{H} \alpha$, que é formado na parte mais externa do envelope) geralmente acompanhadas por linhas fracas de emissão de metais uma vez ionizados (Fe II e, às vezes, $\mathrm{Cr}$ II, Ni II).

$\mathrm{Na}$ década de 80, as observações na região do infravermelho (satélite IRAS) mostraram a existência de uma excesso de fluxo para as estrelas Be. Essas observações levaram à conclusão de que esse excesso de infravermelho é causado pela emissão de radiação livre-livre (free-free ou efeito Bremsstrahlung) proveniente de um envelope gasoso com uma temperatura média $\sim 10^{4} \mathrm{~K}$ e uma densidade eletrônica $\sim 3 \cdot 10^{11} \mathrm{~cm}^{-3}$ com um raio $\sim 4 \mathrm{R} *$ (Gehrz et al.,1974, Underhill 1982). No início das observações no infravermelho não estava claro se o excesso de fluxo era devido à emissão livre-livre realmente ou se era devido à existência de poeira no envelope. As medidas de polarização efetuadas concordaram com os valores acima e forneceram uma forte indicação da existência de um disco circunstelar equatorial (Poeckert \& Marlborough 1976, Underhill 1982). 
As estrelas Be "clássicas" (aquelas que apresentam apenas as características gerais das Be) não emitem na região do rádio. Porém algumas Be's peculiares (Bep's), que mostram raias de emissão interrompidas em seu espectro visível, apresentam emissão rádio; e a explicação dessa emissão requer a presença de envelopes muito grandes ( $\sim$ de centenas de $\mathrm{R}_{*}$ ).

Observações no ultravioleta sugerem a presença de uma componente "quente" $\left(\mathrm{T} \sim 10^{5} \mathrm{~K}\right)$ de gás circunstelar, possivelmente esférica, em complemento à componente equatorial de gás "frio", onde surgem as linhas de emissão e a radiação no infravermelho (Slettebak 1988).

As observações feitas no ultravioleta revelam a presença de raias de absorção de elementos várias vezes ionizados, como por exemplo, as raias de ressonância do C IV, Si IV, N V, e O VI. Esses espectros mostram perfis de tipo P Cygni de estrelas que possuem ventos estelares; a aplicação da teoria dos ventos estelares para as Be leva a valores de perda de massa entre $10^{-11}$ e $10^{-9}$ massas solares por ano e a velocidades da ordem de $10^{3} \mathrm{~km} / \mathrm{s}$. Assim, acredita-se que as regiões polares das estrelas Be apresentam um envelope gasoso quente $\left(\mathrm{T} \sim 10^{5} \mathrm{~K}\right)$, rarefeito e que emerge da estrela a grande velocidade. $\mathrm{Na}$ verdade este vento estelar polar deveria surgir de todas as regiões da estrela, porém a presença do envelope gasoso equatorial mais frio $\left(\mathrm{T} \sim 10^{4}\right.$ $\mathrm{K})$, denso e de baixa velocidade impede a passagem do vento estelar quente através dessa região.

\subsection{O Modelo Rotacional de Struve para o Fenômeno Be}

Em 1931, Otto Struve sugeriu um modelo, baseado nos espectros de emissão no visível, que explica a origem dessas linhas a partir de um envelope gasoso circunstelar, denso e "frio" (em relação à estrela), com a forma de um disco que gira e se estende a partir da região equatorial da mesma. Esse envelope é ionizado pela radiação ultravioleta proveniente da estrela reemitindo-a em outras faixas do espectro quando ocorre a recombinação eletrônica.

A Figura 4 apresenta esquematicamente uma Be segundo o modelo de Struve juntamente com três espectros diferentes relacionados ao ângulo de inclinação $i$ do eixo de rotação estelar em relação à linha de visada do observador. Quando a estrela é vista segundo um pequeno ângulo $i$ (caso A), ocorre uma superposição da linha de absorção fotosférica com a forte emissão proveniente do envelope gasoso, onde não há deslocamento por efeito Doppler. Para $i \sim 90^{\circ}$ temos o caso $\mathrm{C}$ onde a emissão do envelope apresenta uma componente deslocada para o azul e outra para vermelho, devido às regiões do disco aproximando-se e afastando-se do observador respectivamente. A porção do disco que está localizada diretamente entre a Terra e a estrela é responsável pela linha de absorção central, fina e profunda. $\mathrm{O}$ caso $\mathrm{B}$ mostra uma situação intermediária entre os casos A e C. Os casos B e C originam os espectros do tipo Be e Be shell respectivamente mas, conforme discutido acima, uma mesma estrela pode apresentar tanto um como outro tipo de espectro. 


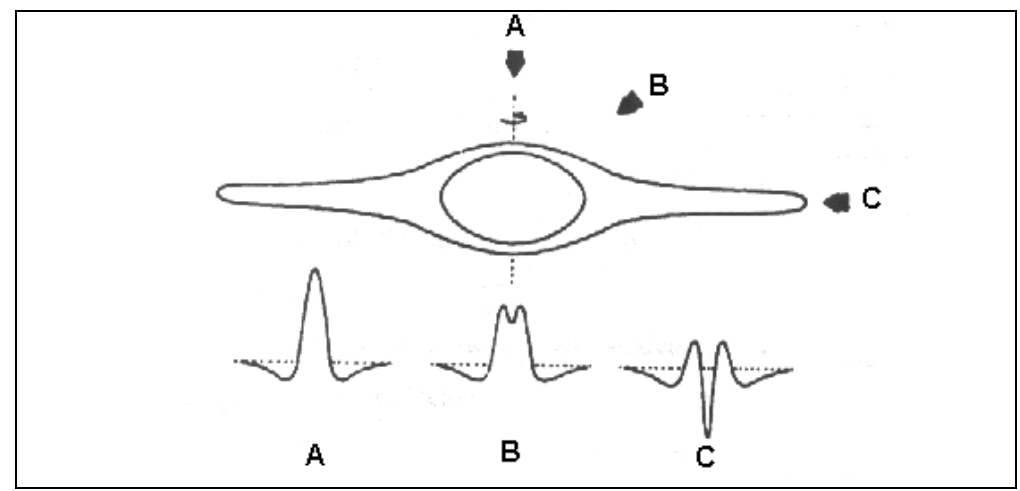

Fig.4. Representação esquemática do modelo rotacional de Struve (Slettebak, 1988).

O modelo de Struve atribui o alargamento das linhas de emissão à rotação do envelope, o qual deve ter um raio da ordem de 3-20 raios estelares $(\mathrm{R} *)$, e densidades da ordem de $10^{11}-10^{12} \mathrm{~cm}^{-3}$. A presença freqüente das raias de emissão do Fe II indica uma temperatura $\sim 10^{4} \mathrm{~K}$ na região de emissão; essa região onde o $\mathrm{Fe} \|$ é produzido é uma região próxima à estrela. As linhas de emissão do hidrogênio são provenientes de regiões cada vez mais externas até chegar ao $\mathrm{H} \alpha$, o qual chega a ser produzido no limite externo do envelope. As velocidades radiais medidas são da ordem de $50-100 \mathrm{~km} / \mathrm{s}$.

Nesse modelo, as Be são consideradas estrelas de rotação rápida pois elas apresentam raias de absorção de origem fotosférica mais largas que do que as estrelas B "normais". De fato, Struve assumiu que a velocidade de rotação deve ser no mínimo igual à velocidade crítica para que o disco gasoso seja formado pela ejeção de matéria devido à rotação rápida. Sabe-se hoje que as Be giram com alta velocidade, porém não com a velocidade crítica de modo que a origem do envelope gasoso ainda não está resolvida completamente. De qualquer forma, somente uma rotação com a velocidade crítica não pode explicar a ejeção de matéria da superfície estelar para grandes distâncias.

Portanto, apesar do modelo de Struve concordar com as observações feitas no visível, infravermelho, rádio e estudos de polarização, ele não explica como o envelope circunstelar é ejetado, uma vez que a estrela não gira com a velocidade crítica. Ao mesmo tempo, as observações feitas no ultravioleta deixam claro que esse modelo não é suficiente para explicar o fenômeno Be.

\subsection{Outros Modelos para o Fenômeno Be}

Como as observações sugerem que o modelo rotacional de Struve não é suficiente para explicar o fenômeno Be, foram propostos outros mecanismos que, em conjunto com a rotação rápida, podem levar a estrela a ejetar o envelope circunstelar (Slettebak 1988). Os principais mecanismos adotados nos modelos que procuram explicar o fenômeno Be são:

1) Ventos estelares, radiativos ou não: podem criar o envelope gasoso e também explicar as observações no ultravioleta;

2) Transferência de matéria num sistema binário: neste caso a Be é uma das componentes do sistema; 
3) Campos magnéticos: podem ejetar o envelope;

4) Oscilações não-radiais: explicam a ejeção do envelope pela troca de energia entre os modos de oscilação e/ou pelo alcance da velocidade crítica devido ao campo de velocidade adicional introduzido pelas oscilações.

Todos os modelos que utilizam um ou mais desses mecanismos físicos explicam alguns dos dados observacionais e não explicam outros, assim todos têm sua parcela de importância e podem ser criticados. Porém, os modelos mais aceitos atualmente são os que adotam as oscilações não-radiais, associados à hipótese de que as Be são estrelas de rotação rápida e que apresentam ventos estelares nas regiões polares.

\subsection{Variações Rápidas nas Estrelas Be}

Além das variações das intensidades dos espectros associadas à ejeção do envelope circunstelar (ciclo Be) com escalas de tempo de anos, existem também variações rápidas (dias ou horas) que são observadas em dados espectroscópicos (variações de perfis de linha - lpv) e fotométricos.

No início dos anos 80 certas distorções com amplitudes $\sim 1 \%$ do contínuo foram observadas movendo-se através de certas linhas de absorção de algumas $\mathbf{B}$ e Be. Essas distorções têm a forma de elevações (bumps) e depressões que se movem pela linha espectral, do azul para o vermelho. Uma das primeiras observações foi realizada por Walker et al. (1979) onde foram detectados bumps movendo-se na linha de absorção fotosférica Hel 667.8nm da estrela $\zeta$ Ophiuchi (HR 6175, HD 149757, $\mathrm{V}=2.56,09.5 \mathrm{Ve}$ ), protótipo das variáveis $\zeta \mathrm{Oph}$.

Os dois principais modelos propostos para a descrição dos bumps observados nas lpv stars são:

1) Modelo rotacional (MR) - Se uma ou mais regiões da fotosfera estelar apresentarem manchas escuras (causadas por exemplo por campos magnéticos) então a falta de absorção na região da mancha será traduzida como uma aparente emissão (bump) na linha espectral (Figura 5a). O fluxo contínuo emitido pela estrela também sofre uma diminuição porém ela não é percebida quando utiliza-se o contínuo normalizado. Como a mancha gira junto com a estrela os bumps terão uma periodicidade igual ou múltipla à freqüência de rotação estelar; 


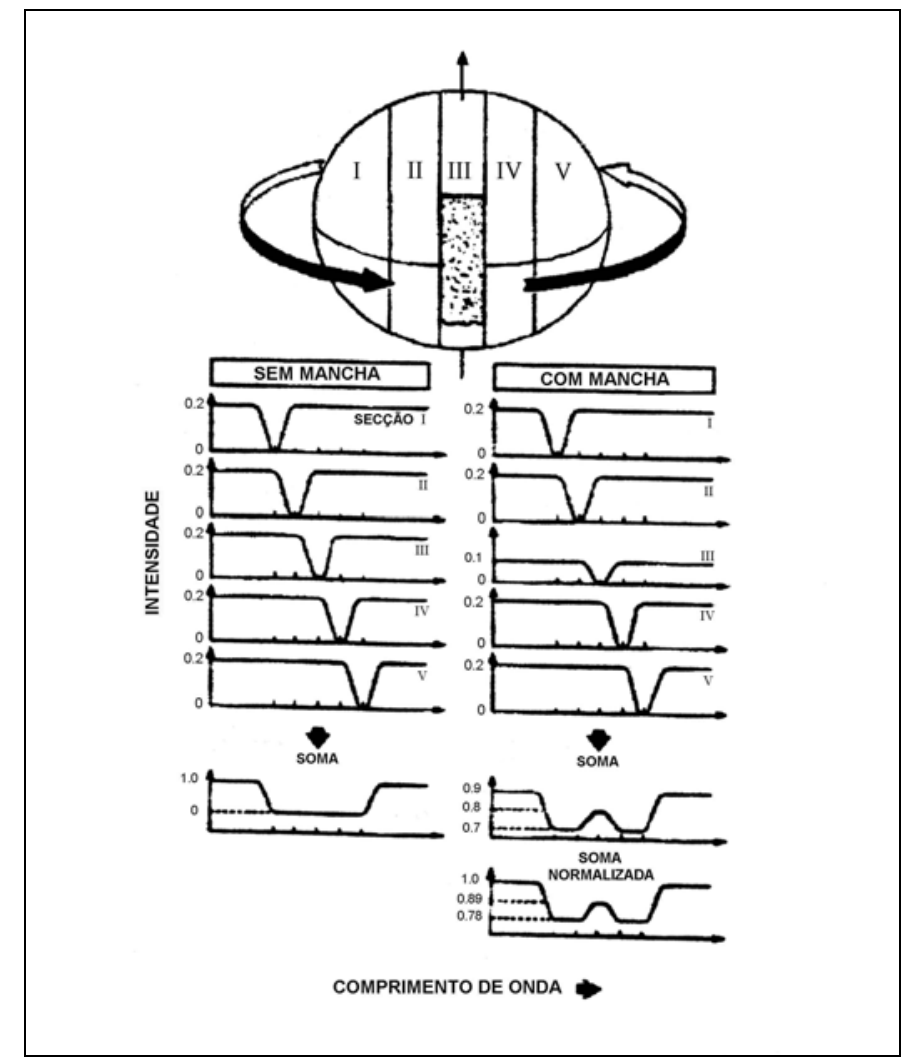

Fig.5a. Modelo de Mancha Fotosférica (adaptado de Vogt et al., 1983)

2) Modelo de pulsações não-radiais (PNR) - as oscilações do gás na fotosfera estelar causam deslocamentos Doppler, variações de temperatura e efeitos geométricos que redistribuem o fluxo na linha espectral deformando-a e gerando bumps com periodicidades que dependem da freqüência da oscilação e da rotação estelar (Figura 5b);

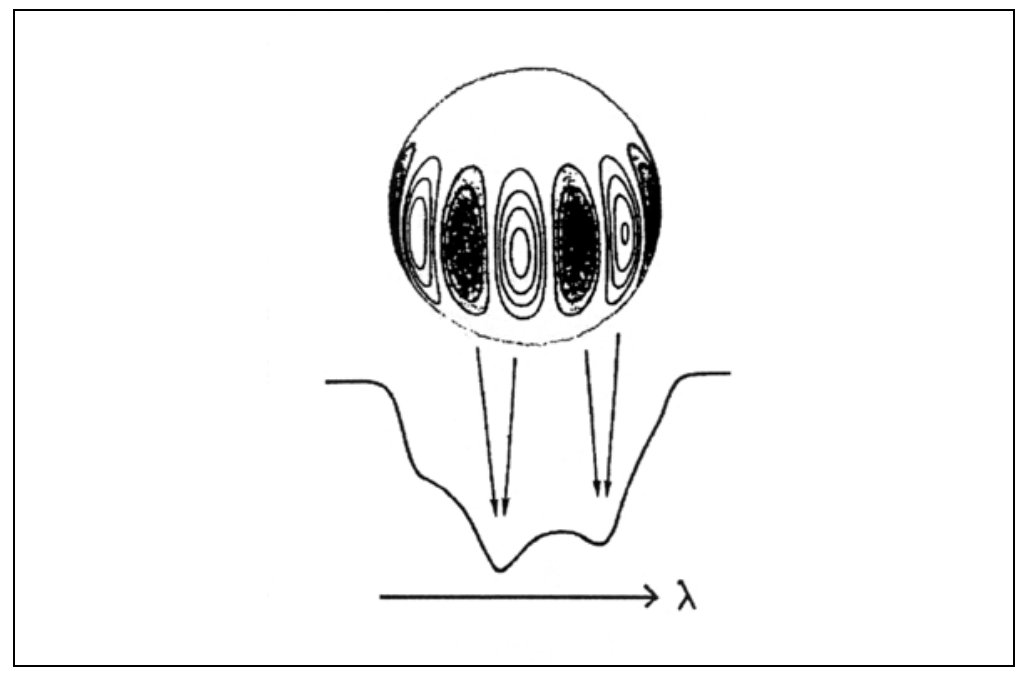

Fig.5b. Modelo de PNR, ilustrado para o caso de modo setorial com $\lambda=|\mathrm{m}|=8$ (Vogt et al., 1983)

Atualmente o modelo de PNR é a hipótese mais aceita para explicar as $l p v$ stars, entretanto, o outro modelo não deve ser descartado, pois é possível a coexistência de outros mecanismos que, atuando em conjunto, produzam as 
variabilidades observadas. Vale notar que estes modelos também podem explicar, satisfatoriamente, as variações fotométricas normalmente observadas nestes objetos.

No caso específico das estrelas tipo Be, as PNR geram, possivelmente, tanto as $l p v$ como o envelope circunstelar (neste caso participando do mecanismo de ejeção de matéria da estrela). 


\section{2- ESTUDO DA ESTRELA $\eta$ CENTAURI}

\subsection{Generalidades}

A estrela $\eta$ Centauri (HD 127972, HR 5440, SAO 225044, MWC 232) é uma estrela Be-Shell brilhante e quente da associação Scorpio-Centaurus, tendo apresentado nas últimas décadas uma variabilidade fotométrica menor que 0.1 mag (Jaschek et al. 1964; Feinstein \& Marraco 1979; Štefl et al. 1994). Essa variabilidade é possivelmente causada por um envelope circunstelar variável, ligado a fases ativas-inativas da estrela, o que explica também as diferentes classificações espectrais (de B1V a B3III, Stefl et al. 1994) encontradas na literatura (Hiltner et al. 1969; Jaschek \& Jaschek 1992; Štefl et al. 1994). Constitui uma estrela variável de tipo $\zeta$ Ophiuchi, cujos membros são caracterizados como estrelas pulsantes de rotação rápida, apresentando $V_{\mathrm{e}} \operatorname{sini} \geq 170 \mathrm{~km} / \mathrm{s}$ (Unno et al., 1989). Assim como as variáveis tipo 53 Persei, as variáveis de tipo $\zeta$ Ophiuchi localizam-se próximas às variáveis $\beta$ Cephei no diagrama HR. Suas principais características são mostradas na tabela seguinte:

\begin{tabular}{|c|c|}
\hline Tipo espectral & B1.5Vne (1) \\
\hline Raio & $\begin{array}{l}4.3-4.9 \quad(2) \\
6.0-6.8 \quad(3)\end{array}$ \\
\hline Massa & $\begin{array}{c}8.6-11.0 \\
10.3-10.4\end{array}$ \\
\hline $\mathrm{T}_{\text {efetiva }}$ & $22500 \mathrm{~K}$ \\
\hline $\mathrm{V}_{\mathrm{e}} \operatorname{sini}$ & $350 \mathrm{~km} \cdot \mathrm{s}^{-1}$ (5) \\
\hline $\mathrm{V}$ & $2 .^{\mathrm{m}} 3-2 .^{\mathrm{m}} 4 \quad(6)$ \\
\hline$B-V$ & $-0 .{ }^{\mathrm{m}} 19 \quad(6)$ \\
\hline U-B & $-0 .^{\mathrm{m}} 82 \quad(6)$ \\
\hline$\alpha(\mathrm{J} 2000)$ & $14^{\mathrm{h}} 35^{\mathrm{m}} 30^{\mathrm{s}} .42$ \\
\hline$\delta(\mathrm{J} 2000)$ & $-42^{\circ} 09^{\prime} 28^{\prime} .2$ \\
\hline Coord. galáticas & $322.77^{\circ}+16.67^{\circ}$ \\
\hline
\end{tabular}

Tab 2. Principais características físicas da estrela $\eta$ Centauri. Referências:

(1) CDS - Strasbourg; (2) Harmanec, 1988; (3) Underhill, 1982;

(4) Dachs et al., 1988; (5) Hanuschik et al, 1996; (6) Johnson et al, 1966. 


\subsection{Instrumentação e Dados Observacionais}

Foram utilizados neste trabalho dados espectroscópicos de $\eta$ Centauri obtidos no MCT/LNA e dados fotométricos obtidos com o satélite HIPPARCOS. Os dados espectroscópicos foram obtidos com telescópio $\mathrm{B} \& \mathrm{C}$ de $1.60 \mathrm{~m}$ (foco coudé) equipado com uma câmara EMI CCD de 1152 x 770 pixels. A fenda do espectrógrafo foi fixada em $250 \mu \mathrm{m}$ e centrada na linha fotosférica Hel $\lambda 667.8 \mathrm{~nm}$, com uma rede de $1800 \mathrm{l} / \mathrm{mm}\left(1^{\mathrm{a}}\right.$ ordem inversa). A relação sinal/ruído dos espectros assume valores tipicamente entre 100 e 300, com tempos de exposição da ordem de 3 a 5 minutos. Os espectros apresentam uma resolução espectral $(\lambda / \Delta \lambda)$ de aproximadamente 60000 e dispersão recíproca de 0.08 Å/pixel.

As observações espectroscópicas foram reduzidas utilizando-se o pacote IRAF (Image Reduction and Analysis Facility - NOAO) utilizando-se técnicas padrão para espectros $C C D$ bidimensionais e suas características principais estão resumidas na Tabela 3.

\begin{tabular}{ccccc}
\hline Data & $\begin{array}{c}\text { HJD } \\
(2449788 \text { dias }+)\end{array}$ & $\begin{array}{c}\mathrm{N}^{\mathrm{o}} \\
\text { espectros } \\
667.8 \mathrm{~nm}\end{array}$ & $\begin{array}{c}\text { No } \\
\text { espectros } \\
656.3 \mathrm{~nm}\end{array}$ & $\begin{array}{c}\text { Resolução } \\
\text { em } \\
\text { freqüência } \\
\text { (ciclos/dia) }\end{array}$ \\
\hline $09 / 03 / 1995$ & 0.680 a 0.773 & 21 & ---- & 10.75 \\
$12 / 03 / 1995$ & 1.692 a 1.772 & 21 & ---- & 12.5 \\
$15 / 03 / 1995$ & 4.662 a 4.745 & 44 & ---- & 12.05 \\
$17 / 03 / 1995$ & 6.649 a 6.748 & 104 & ---- & 10.1 \\
$18 / 03 / 1995$ & 7.650 a 7.736 & 19 & ---- & 11.63 \\
$03 / 05 / 1996$ & 419.483 a 419.711 & 49 & 1 & 4.39 \\
$04 / 05 / 1996$ & 420.431 a 420.671 & 66 & ---- & 4.17 \\
$05 / 05 / 1996$ & 421.430 a 421.642 & 247 & 1 & 4.72 \\
$30 / 05 / 1996$ & 446.608 a 446.770 & 48 & ---- & 6.17 \\
$31 / 05 / 1996$ & 447.650 a 447.764 & 63 & ---- & 8.77 \\
$01 / 06 / 1996$ & 448.649 a 448.738 & 33 & ---- & 11.24 \\
$02 / 06 / 1996$ & 449.593 a 449.679 & 33 & ---- & 11.63 \\
$23 / 05 / 1997$ & 804.568 a 804.754 & 7 & 1 & 5.38 \\
$25 / 05 / 1997$ & ---- & ---- & 3 & ---- \\
$26 / 05 / 1997$ & 807.466 a 807.751 & 8 & 1 & 3.51 \\
$27 / 05 / 1997$ & 808.425 a 808.764 & 11 & ---- & 2.95 \\
$09 / 06 / 1998$ & 1186.406 a 1186.489 & 13 & 3 & 12.05 \\
$10 / 06 / 1998$ & 1187.471 a 1187.653 & 18 & 1 & 5.49 \\
TOTAL & $\mathbf{0 . 6 8 0}$ a $\mathbf{1 1 8 7 . 6 5 3}$ & $\mathbf{8 0 5}$ & $\mathbf{1 1}$ & $\mathbf{0 . 0 0 0 8 4}$ \\
\hline
\end{tabular}

Tab. 3. Observações espectroscópicas de $\eta$ Cen obtidas no MCT/LNA entre 09/março/95 e 10/junho/98.

Neste trabalho, os espectros referentes ao ano de 1995 já foram analisados em publicação anterior (Janot et al., 1999), e os resultados obtidos desse artigo serão usados para efetuar uma comparação com os resultados do presente trabalho. Dessa forma, será feita apenas a análise temporal dos resíduos dos espectros de 1996, 1997 e 1998. Os 
dados de 1995 serão incluídos apenas no estudo das variabilidades V/R. Os dados fotométricos utilizados neste trabalho foram obtidos pelo satélite HIPPARCOS ao longo dos anos de 1990, 1991 e 1992.

A Figura 6 mostra os espectros janela (window spectra) dos dados espectroscópicos de 1996, 1997/1998, e 1996/1997/1998. Assim, cada sinal periódico eventualmente presente nos dados aparecerá convoluído com sua janela espectral correspondente, de modo que uma boa interpretação das análises temporais necessita do conhecimento da forma do espectro janela dos dados analisados. Em todos os casos mostrados, nota-se que a principal freqüência da janela é a de $1 \mathrm{c} / \mathrm{d}$ e seus múltiplos, o que reflete a periodicidade dia/noite das observações.

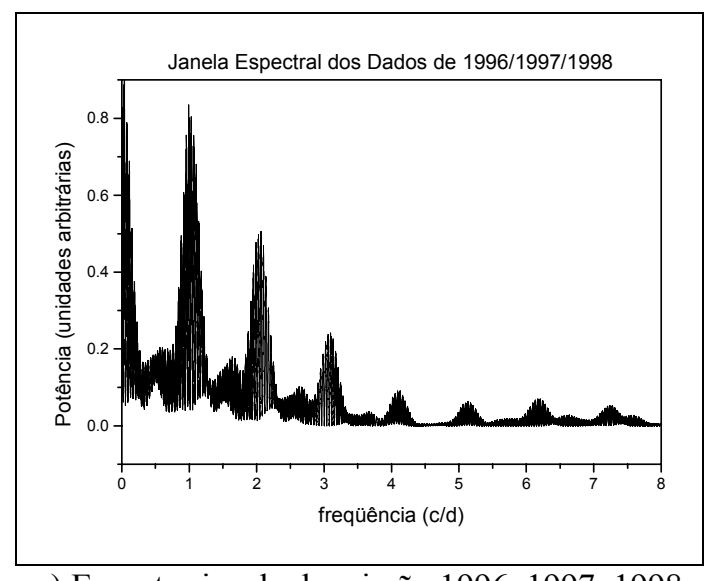

a) Espectro janela da missão 1996, 1997, 1998

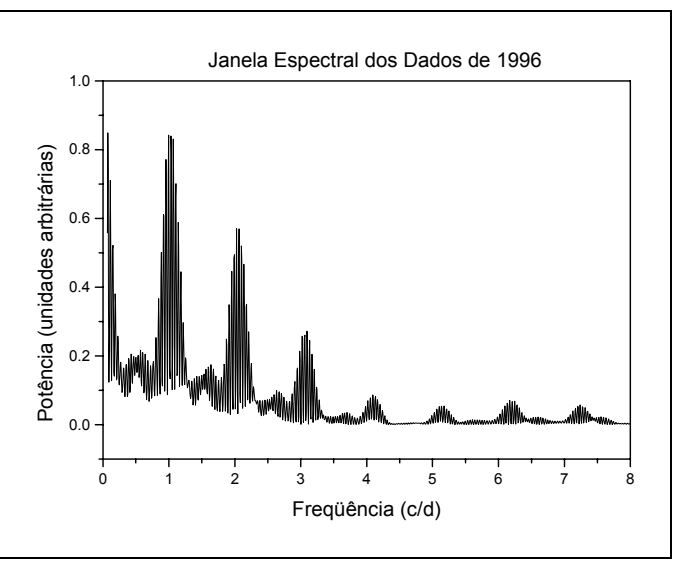

c) Espectro janela da missão 1996

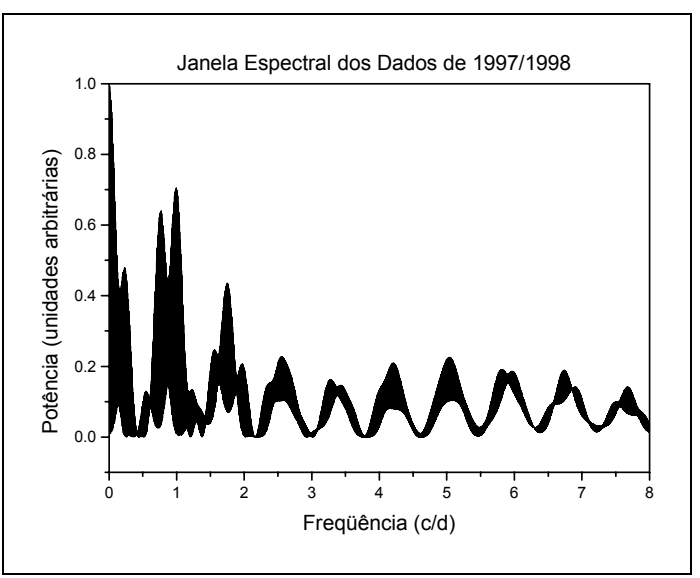

b) Espectro janela da missão 1997, 1998

Fig.6. Espectros janela (window spectra) dos dados espectroscópicos referentes aos conjuntos de dados de 1996/1997/1998 (Fig.6a), 1997/1998 (Fig.6b) e 1996 (Fig.6c).

A seguir, ilustramos os perfis de linha dos espectros centrados em Hel $\lambda 667.8 \mathrm{~nm}$ e em $\mathrm{H} \alpha \lambda 656.3 \mathrm{~nm}$ mostrados na tabela 3. Os perfis em $\mathrm{H} \alpha \lambda 656.3 \mathrm{~nm}$ mostrados nas Figuras 7d, 7e e 7f são os perfis médios para cada ano (1996, 1997 e 1998, 
respectivamente). Comparando-se as Figuras $7 \mathrm{~d}, 7 \mathrm{e}$ e $7 \mathrm{f}$, vemos em $7 \mathrm{~d}$ o término de um período de quiescência seguido por um aumento significativo na emissão do $\mathrm{H} \alpha$, o que é um indicativo do aumento da atividade da estrela nesse período. O seu período de atividade, determinado a partir da variabilidade da emissão em $H \alpha$, é de aproximadamente 10 anos (Hanuschik et al., 1996) conforme a Figura 8. Repare que os espectros de $\mathrm{H} \alpha$ mostrados nas Figuras 7d, 7e e $7 \mathrm{f}$ se inserem bem nesse contexto, uma vez que o último período de atividade de $\eta$ Cen ocorreu em 1987, e o novo período se iniciou em 1997. 


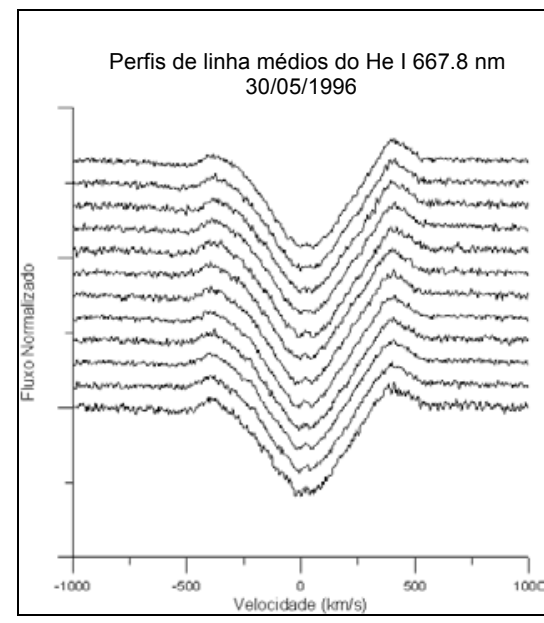

a) Perfis médios de 30/05/1996

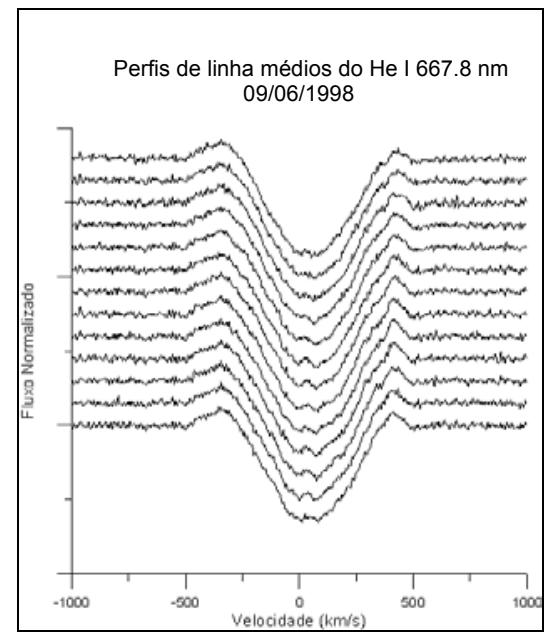

b) Perfis médios de 09/06/1998

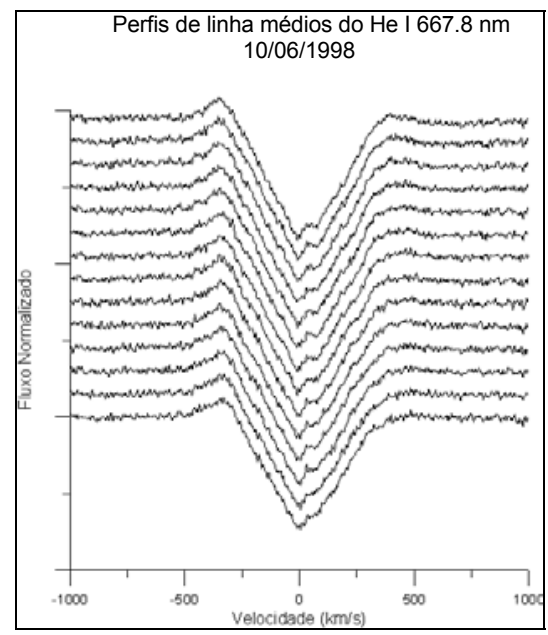

c) Perfis médios de 10/06/1998

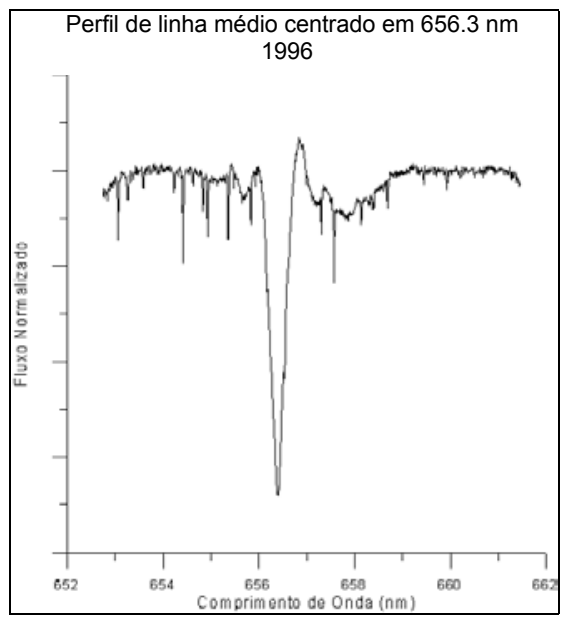

d) $\mathrm{H} \alpha$ médio de 1996

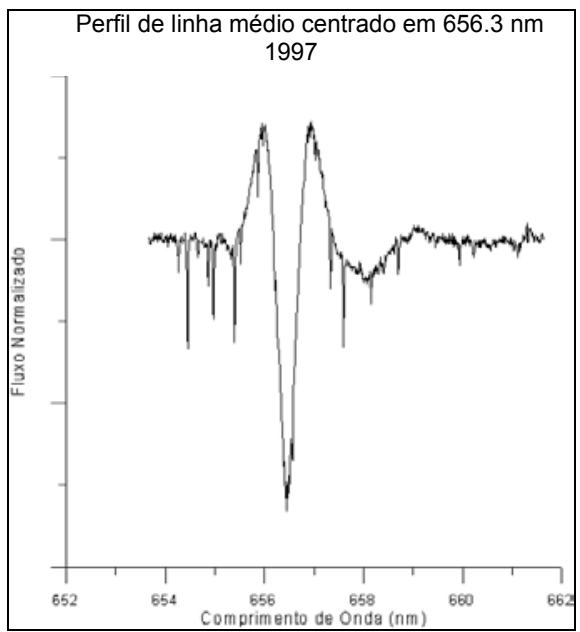

e) $\mathrm{H} \alpha$ médio de 1997

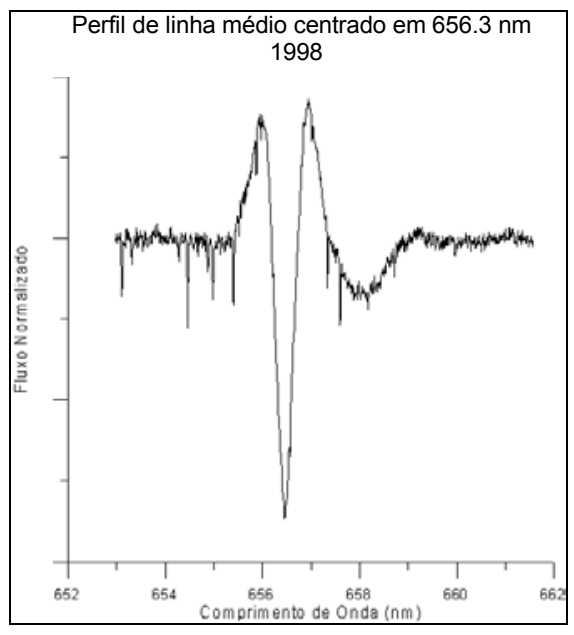

f) H $\alpha$ médio de 1998

Fig.7. Espectros médios de $\eta$ Cen centrados em Hel $\lambda 667.8 \mathrm{~nm}$ e em Ha $\lambda 656.3 \mathrm{~nm}$. 


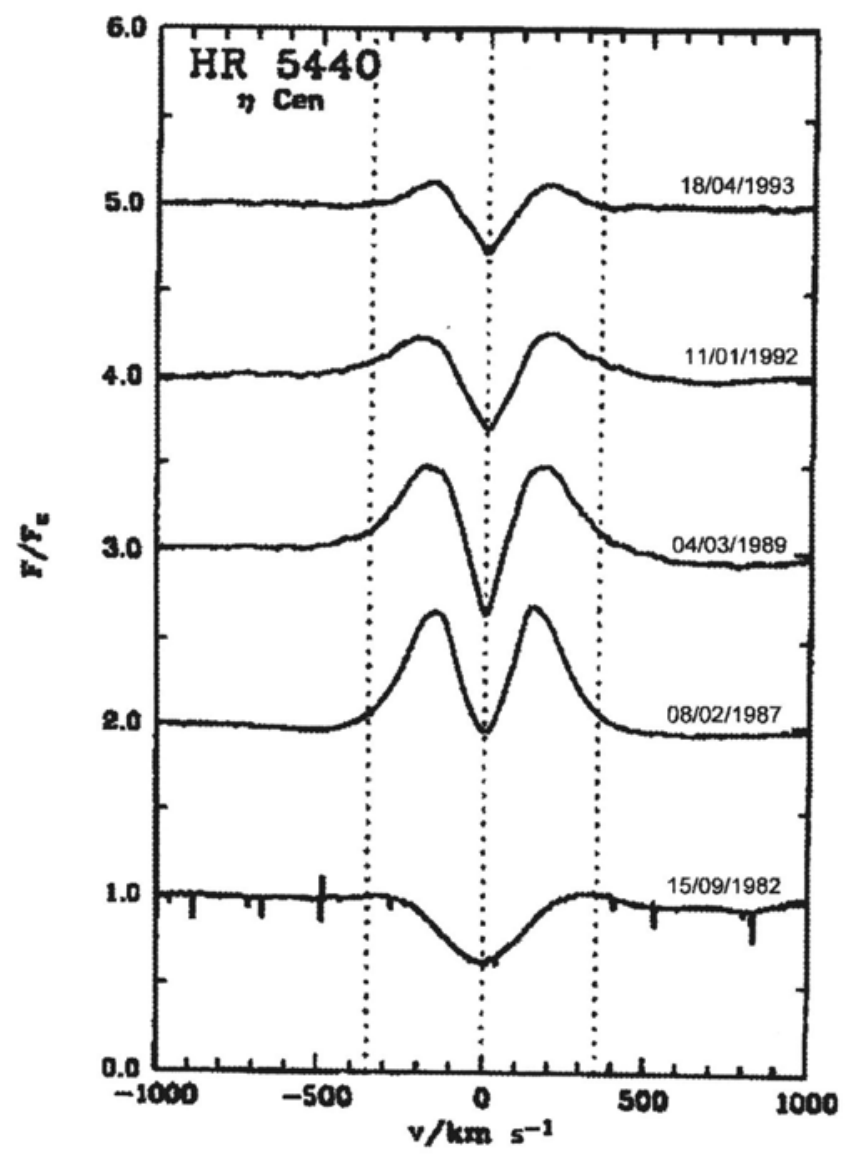

Fig.8. Perfis médios anuais de $\mathrm{H} \alpha \lambda 656.3 \mathrm{~nm}$. Note que o período de atividade da estrela é de cerca de 10 anos (adaptado de Hanuschik et al., 1996).

A partir das Figuras 7a, $7 \mathrm{~b}$ e 7c, podemos ainda identificar duas peculiaridades do perfil em Hel $\lambda 667.8 \mathrm{~nm}$, que são as variações nas intensidades das asas azul e vermelha da linha e a presença de um pico central em quase emissão (CQE - Central QuasiEmission Peak) que parece estar relacionado com um efeito de rotação diferencial da estrela (Zorec, 1988) o qual será visto mais adiante.

Comparando-se as Figuras $7 \mathrm{a}, 7 \mathrm{~b}$ e $7 \mathrm{c}$, vemos com clareza uma alternância nas intensidades das asas azul e vermelha dos perfis em $\lambda 667.8 \mathrm{~nm}$, isto é, à medida em que a asa azul da linha aumenta de intensidade, a vermelha diminui. Esse fenômeno já foi observado anteriormente em outras estrelas Be, como $\alpha$ Ara, $\delta$ Cen e 48 Lib no comprimento de onda do Fe II $\lambda 531.7 \mathrm{~nm}$, uma vez que esse perfil é opticamente fino e dessa forma os efeitos cinemáticos do disco não se misturam com os efeitos de

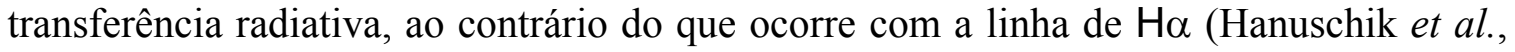
1995). Mais adiante será feita uma abordagem no sentido da obtenção das periodicidades das variabilidades nas asas da linha em He I e na posição dos $C Q E$ 's em velocidade. 


\subsection{Tratamento dos Dados}

Os perfis espectrais descritos na Tabela 3 foram tratados com o pacote IRAF (Image Reduction and Analysis Facility) distribuído pelo $N O A O$, o qual é operado pela Association of Universities for Research in Astronomy - AURA, Inc., sob acordo cooperativo com a National Science Foundation.

Em princípio, os espectros provenientes do LNA são arquivos com formato FITS. O primeiro passo na redução de dados é converter esses arquivos para o formato do IRAF (*.pix e *.imh, que são as imagens e seus cabeçalhos, respectivamente) usando as tasks rfits ou nfits (esta última no caso de arquivos "swapados"). Em seguida, recortar todas as imagens com tamanho apropriado (task cedproc), fazer a média de todos os bias (exposições de "tempo zero" de integração, entre $50 \mathrm{~ms}$ e $1 \mathrm{~s}$ ) para obter uma estimativa do nível do ruído eletrônico intrínseco (task darkcombine). Então, após subtrair a média dos bias de cada um dos flat fields através do ccdproc, fazer a média de todos os flat fields de cúpula (que são exposições de uma lâmpada incandescente ou de uma tela branca iluminada por uma luz branca uniforme) com a task flatcombine a fim de obter uma estimativa das variações pixel a pixel da sensibilidade do $C C D$, bem como de possíveis efeitos de franjas de difração sobre a imagem. Após essa tarefa, deve-se subtrair o bias médio de todos os espectros e dividir cada um deles pelo flat field médio (task ccdproc). Em seguida, fazer o tratamento dos espectros da lâmpada de Th-Ar (ccdproc), depois extrair os espectros com a task apall (estelares e de lâmpadas de calibração), isto é, transformar os espectros bidimensionais do $C C D$ em espectros unidimensionais (contagens x pixels) através de uma média ponderada das contagens na direção espacial. Nesta etapa, pode-se definir alguns parâmetros:

a) o ajuste das contagens de fundo do $C C D$;

b) o limite mínimo das contagens que podem ser consideradas na soma que resultará no espectro unidimensional;

c) a função usada na busca dos máximos de contagens ao longo da dispersão (pois em geral o espectro da estrela não está totalmente alinhado com o $C C D$ ). Como os espectros da lâmpada de Th-Ar aparecem como linhas de emissão de igual intensidade, é necessário fornecer um espectro bidimensional de referência para que o espectro da lâmpada seja extraído seguindo a mesma curva dentro do $C C D$.

Logo após essa fase, deve-se identificar as linhas de emissão da lâmpada de calibração e também construir uma curva ( $\lambda$ x pixel) que será usada na calibração dos espectros estelares (task identify). Para a lâmpada de Th-Ar, o IRAF já possui uma biblioteca com as linhas mais importantes, de modo que é necessário apenas apontar algumas linhas com o cursor, e as demais serão indicadas automaticamente. Para efetuar uma boa calibração, é necessário que o rms (root mean square) do ajuste seja pelo menos 5 vezes menor que a dispersão recíproca do espectro. Caso isto não ocorra, devemos eliminar os pontos que afetam o ajuste.

Durante as observações, podem ocorrer variações nas posições das linhas espectrais sobre o $C C D$, então é costume realizar-se um conjunto de medidas da lâmpada de calibração durante a noite. Necessita-se então fazer um ajuste temporal (interpolação) dos 
diversos espectros de referência (em geral são dois) com a task refspectra, o que possibilita uma calibração dos espectros estelares levando em conta as citadas variações.

Após calcular a data juliana $(J D)$ e a data juliana heliocêntrica (HJD) com a task setjd, pode-se converter os eixos horizontais dos espectros de pixel para comprimento de onda $(\AA)$ através da task dispcor. Ainda com essa task, pode-se redimensionar os espectros de forma a que todos contenham o mesmo número de pixels, para tanto deve-se fornecer os valores de $\lambda$ inicial, do passo $\Delta \lambda$ e do número de pixels desejado, o que possibilita construir as séries temporais num mesmo comprimento de onda $\lambda$.

Em seguida, deve-se mudar o referencial do observador para o Local Standard of Rest - LSR por meio da task rvcor, corrigindo os espectros do movimento do centro da Terra em relação ao baricentro Terra-Lua, do movimento do baricentro Terra-Lua em relação ao centro do Sol e do movimento do Sol em relação ao $L S R$. A task rvcor atualiza os headers das imagens com a velocidade do $L S R(V L S R)$ e com a velocidade heliocêntrica (VHELIO). A correção efetiva dos espectros para o $L S R$ é realizada em seguida com a task dopcor.

Finalmente, a visualização e a normalização dos espectros é realizada através da task splot. A determinação do contínuo é feita com a task continuum, a conversão dos espectros resultantes para formato $A S C I I$ é feita com a task wspectext, e a confecção dos resíduos é feita a partir dos espectros da estrela com imarith. 


\section{3- TEORIA DAS PULSAÇÕES NÃO RADIAIS}

\subsection{Possíveis mecanismos de excitação}

Os mecanismos de excitação das pulsações não radiais em estrelas variáveis constituem até hoje uma questão em aberto. Dessa forma, vários modelos foram propostos, dependendo da massa da estrela, sua posição no diagrama HR, da abundância de elementos e presença de rotação rápida. Devido à complexidade do tema, não faremos aqui uma abordagem matemática, dando preferência simplesmente a uma descrição sucinta dos modelos atualmente aceitos. Os principais mecanismos atuantes em estrelas variáveis ao longo do diagrama HR são o mecanismo $\kappa$ e o mecanismo $\varepsilon$.

1) Mecanismo $\kappa$ : caracterizado por variações abruptas na opacidade da zona de ionização, o que impede a livre passagem do fluxo radiativo e a energia excedente é convertida em energia de oscilação Para uma estrela com envelope externo em equilíbrio, a luminosidade $\mathrm{L}_{\mathrm{R}}$ é constante e o mecanismo- $\kappa$ desencadeará uma oscilação se $\frac{d}{d r}\left(\kappa_{T}+\frac{\kappa_{\rho}}{\Gamma_{3}-1}\right)>0$, onde $\kappa_{T}=\left(\frac{\partial \ln \kappa}{\partial \ln T}\right)_{\rho}$ representa a dependência da opacidade com a temperatura a densidade constante, $\kappa_{\rho}=\left(\frac{\partial \ln \kappa}{\partial \ln \rho}\right)_{T}$ é a variação da opacidade com a densidade a temperatura constante, e $\Gamma_{3}=\left(\frac{\partial \ln T}{\partial \ln \rho}\right)_{S}+1$ é o expoente adiabático (Unno et al., 1989). Se uma região no envelope estelar satisfizer essa condição, então o fluxo radiativo proveniente do interior da estrela é bloqueado pelo aumento da opacidade, que é função da temperatura e da densidade. $\mathrm{O}$ valor de $\kappa_{\mathrm{T}}$ aumenta na parte interna da zona de ionização e decresce na parte externa. Assim, as regiões de excitação e amortecimento devidas ao mecanismo- $\kappa$ estão localizadas, respectivamente, nas partes interna e externa da zona de ionização. Em particular, constatou-se que estrelas de tipo $\beta$ Cephei apresentam instabilidades pulsacionais para modos radiais bem como modos não radiais de baixo grau (Cox et al., 1992; Moskalik et al., 1992). Essas instabilidades são sensíveis à abundância de elementos pesados: as instabilidades são maiores para abundâncias maiores (e portanto opacidades maiores). Quando uma pequena perturbação faz a temperatura aumentar localmente, a opacidade aumenta e ocorre a transferência de energia radiativa em energia pulsacional. Inversamente, quando a temperatura diminui, a opacidade diminui também e ocorre uma transferência inversa. Esse mecanismo, conhecido como mecanismo- $\kappa$, tem por efeito aumentar as amplitudes das perturbações até que os efeitos não lineares se tornem importantes e cessem esse crescimento.

2) Mecanismo $\varepsilon$ : caracterizado por variações abruptas na taxa de geração de energia nuclear devido a variações de temperatura, onde o excedente de energia é convertido 
em energia de oscilação. Esse mecanismo é mais forte para reações nucleares com grande sensibilidade à temperatura, como em reações p-p;

A seguir, vemos alguns exemplos de possíveis mecanismos de excitação de oscilações e os principais objetos do diagrama HR associados.

Mecanismo de Excitação Objetos relacionados

\begin{tabular}{cc}
\hline $\begin{array}{c}\text { Mecanismo } \kappa \text { na zona de ionização } \\
\text { do H }\end{array}$ & $\begin{array}{c}\text { Variáveis ZZ Ceti (DA) } \\
\text { Cefeidas }\end{array}$ \\
$\begin{array}{c}\text { Mecanismo } \kappa \text { na zona de ionização } \\
\text { do He }\end{array}$ & Variáveis DB
\end{tabular}

Mecanismo $\varepsilon$

Estrelas massivas e supermassivas

Anãs massivas de índice politrópico 1.5

Wolf-Rayet (pulsações radiais)
Mecanismo $\kappa$ na zona de ionização do C
Estrelas DOV
(núcleos de nebulosas planetárias e estrelas pré anãs-brancas muito quentes)

Tabela 4. Alguns exemplos de mecanismos de excitação de oscilações radiais e não radiais, apresentando os principais objetos do diagrama HR associados (Unno et al., 1989) 


\subsection{Caracterização dos Parâmetros $\ell$ e $m$}

Como as amplitudes das oscilações são relativamente pequenas se comparadas com o diâmetro estelar, podemos tratá-las como perturbações em um estado de equilíbrio esférico. Negligenciando-se os efeitos da rotação da estrela ou de campos magnéticos e. assumindo que o estado não perturbado está em equilíbrio independente do tempo, então as perturbações das variáveis físicas são proporcionais a $Y_{\ell}^{m}(\theta, \phi) e^{i \sigma t}$, onde $Y_{\ell}^{m}(\theta, \phi)$ são os harmônicos esféricos, $\theta$ é a colatitude, $\phi$ é o ângulo azimutal, $\sigma$ é a freqüência angular e t o tempo. A função $Y_{\ell}^{m}(\theta, \phi)$ é expressa explicitamente como:

$$
Y_{\ell}^{\mathrm{m}}(\theta, \phi)=\mathrm{N}_{\ell}^{\mathrm{m}} \mathrm{P}_{\ell}^{|\mathrm{m}|}(\cos \theta) e^{i m \phi}
$$

onde $\mathrm{P}_{\ell}^{\mid \mathrm{m}}(x)$ denota os polinômios associados de Legendre de grau $\ell$ e ordem $\mathrm{m}$, onde $\ell(=0,1,2, \ldots)$ e m $(=-\ell,-\ell+1, \ldots, 0, \ldots, \ell-1, \ell)$ são inteiros, e $\mathrm{N}_{\ell}^{\mathrm{m}}$ é a constante de normalização.

Adotando a notação $\xi_{n} \ell^{(\mathrm{r})}$ para representar as perturbações radiais, onde $\mathrm{n}=1$, $2, \ldots$, então pode-se descrever um modo próprio de oscilação da estrela (modo normal) através de três quantidades, $\mathrm{n}, \ell$ e m:

$$
\xi_{n \ell \mathrm{m}}(\mathrm{r}, \theta, \phi, \mathrm{t})=\xi_{n \ell}(\mathrm{r}) Y_{\ell}^{\mathrm{m}}(\theta, \phi) e^{i \sigma} n \ell \mathrm{t}
$$

Assim, a perturbação total em um ponto da estrela é a soma das perturbações introduzidas por cada modo próprio de oscilação:

$$
\xi_{n \ell \mathrm{m}}(\mathrm{r}, \theta, \phi, \mathrm{t})=\sum_{n \ell m} \xi_{n \ell}(\mathrm{r}) Y_{\ell}^{\mathrm{m}}(\theta, \phi) e^{i \sigma} n \ell{ }^{\mathrm{t}}
$$

Vamos ver o significado físico dos parâmetros pulsacionais:

$\rightarrow \mathrm{n}$ (ordem radial): corresponde ao número de nodos que satisfazem à relação $\xi_{n \ell}\left(r_{i}\right)=0$, onde $\mathrm{i}=1,2,3 \ldots, \mathrm{n}$ representam o número de pontos não perturbados ao longo do raio da estrela.

$\rightarrow \ell$ (grau): corresponde ao número total de planos nodais que cortam a superfície da estrela.

$\rightarrow m$ (ordem azimutal): corresponde ao número de planos nodais que cortam perpendicularmente o equador da estrela. 
A Figura 9 mostra o aspecto da superfície de uma estrela em um dado instante para diferentes valores de $\ell$ e m:

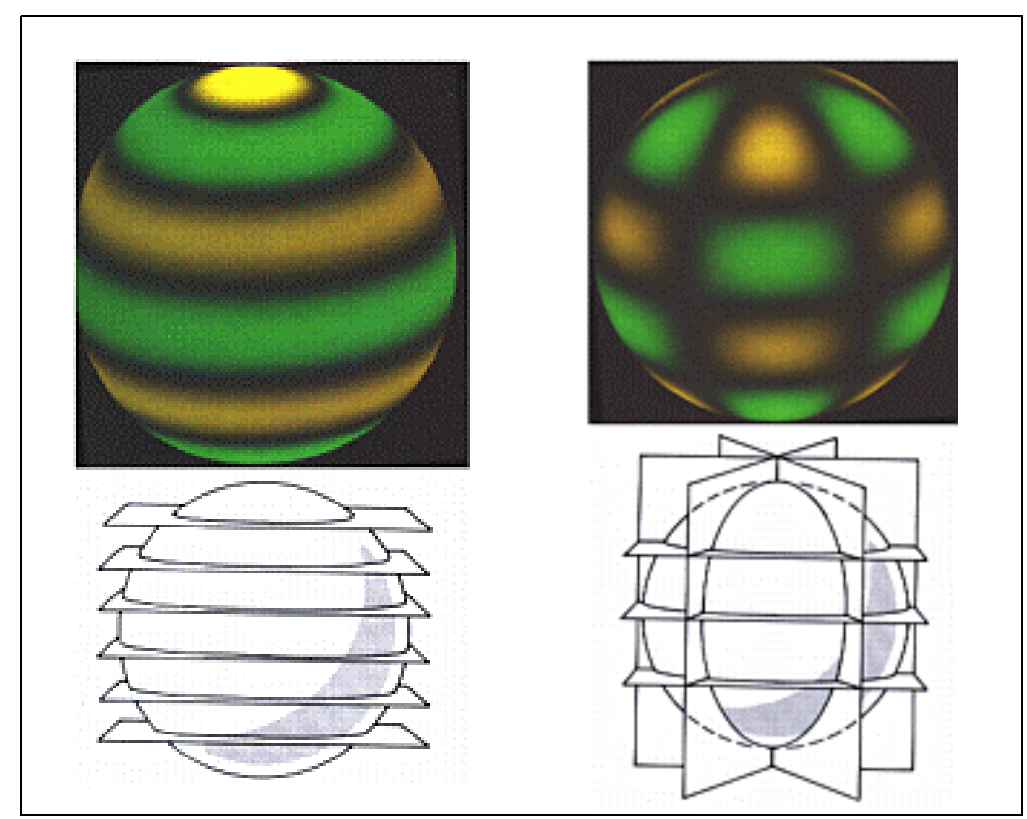

Fig.9. Padrões modais de oscilações não radiais para 2 modos normais de grau $\ell=6$ e ordens $m=0$ (esquerda) e $m=3$ (direita) (adaptado de Harvey et al., 1987).

\subsection{Equações Básicas das PNR’s}

Os movimentos oscilatórios de uma estrela em torno de sua posição de equilíbrio satisfazem as equações da hidrodinâmica (Unno et al. 1989). As equações básicas são as de conservação da massa (ou equação da continuidade), momentum, energia e potencial gravitacional (equação de Poisson):

$$
\begin{gathered}
\frac{\partial \rho}{\partial \mathrm{t}}+\vec{\nabla} \cdot(\rho \overrightarrow{\mathrm{u}})=0 \quad \text { (continuidade) } \\
\rho\left(\frac{\partial}{\partial \mathrm{t}}+\overrightarrow{\mathrm{u}} \cdot \vec{\nabla}\right) \overrightarrow{\mathrm{u}}=\rho \overrightarrow{\mathrm{f}}+\operatorname{div} \mathfrak{I}-\vec{\nabla} \mathrm{P}-\rho \vec{\nabla} \phi \quad \text { (momentum) } \\
\rho \mathrm{T}\left(\frac{\partial}{\partial \mathrm{t}}+\overrightarrow{\mathrm{u}} \cdot \vec{\nabla}\right) \mathrm{S}=\rho\left(\varepsilon_{\mathrm{n}}+\varepsilon_{\mathrm{v}}\right)-\vec{\nabla} \cdot \overrightarrow{\mathrm{F}} \quad \text { (energia) } \\
\vec{\nabla}^{2} \phi=4 \pi \mathrm{G} \rho \quad \text { (Poisson) }
\end{gathered}
$$


onde $\mathrm{P}$ é a pressão, $\rho$ a densidade de massa, T a temperatura, $\mathrm{S}$ a entropia específica, $\overrightarrow{\mathrm{u}} \mathrm{a}$ velocidade do fluido (incluindo movimentos convectivos), $\overrightarrow{\mathrm{f}}$ as forças eletromagnéticas e externas, $\mathfrak{I}$ tensor de viscosidade, $\varepsilon_{\mathrm{n}}$ e $\varepsilon_{\mathrm{v}}$ são as taxas de geração de energia, por unidade de massa, nuclear e térmica pela viscosidade, respectivamente (a energia levada pelos neutrinos não é considerada), $\overrightarrow{\mathrm{F}}$ o fluxo de energia total, $\phi$ o potencial gravitacional e $\vec{\nabla}$ o operador gradiente. Vamos agora descrever as PNR's apoiados nas seguintes hipóteses:

1) a estrela não está sujeita a rotação (a qual será considerada mais tarde), campo magnético e forças externas;

2) ausência de zonas convectivas e condutivas;

3) a estrela é considerada como um fluido ideal (não viscoso);

4) utilizaremos a teoria de perturbação de $1^{\mathrm{a}}$ ordem aplicada às equações básicas da estrutura estelar. Como estas equações também são lineares as oscilações podem ser decompostas em modos normais independentes entre si;

5) a estrela não perturbada é estática e com simetria esférica.

Dessa forma, os termos $\overrightarrow{\mathrm{f}}, \varepsilon_{\mathrm{v}}$ e $\operatorname{div} \mathfrak{I}$ que aparecem nas equações acima são nulos de modo que a expressão (3.5) se torna a equação de Euler. No caso de ausência de movimentos turbulentos convectivos é comum representar-se o campo de velocidade pela variável $\overrightarrow{\mathrm{v}}$ ao invés de $\overrightarrow{\mathrm{u}}$. Além disso, todas as grandezas físicas acima são, em geral, funções da posição $\vec{r}$ e do tempo $t(\rho=\rho(\vec{r}, t)$, ...etc $)$. Em coordenadas esféricas podemos representar por $\overrightarrow{\mathrm{r}}=(\mathrm{r}, \theta, \varphi)$.

Pela hipótese de ausência de convecção e condução o fluxo de energia será apenas radiativo e dado por

$$
\overrightarrow{\mathrm{F}}=\overrightarrow{\mathrm{F}}_{\mathrm{Rad}}=-\left(\frac{4 \mathrm{ac}}{3 \mathrm{k}} \mathrm{T}^{3}\right) \vec{\nabla} \mathrm{T} \equiv-\mathrm{K} \vec{\nabla} \mathrm{T} \quad \text { (transporte radiativo) }
$$

onde a é a constante da densidade de radiação, c a velocidade da luz e k um coeficiente de absorção médio (por exemplo a média de Rosseland).

A partir de considerações termodinâmicas (Unno et al., 1989) podemos chegar também a

$$
\frac{\delta \rho}{\rho}=\frac{1}{\Gamma_{1}} \frac{\delta \mathrm{P}}{\mathrm{P}}-\nabla_{\mathrm{ad}} \frac{\rho \mathrm{T}}{\mathrm{P}} \delta \mathrm{S}
$$

e às relações termodinâmicas:

$$
\begin{gathered}
\Gamma_{1}=\left(\frac{\partial \ln \mathrm{P}}{\partial \ln \rho}\right)_{\mathrm{S}} \quad\left(1^{\mathrm{o}} . \text { expoente adiabático }\right) \\
\nabla_{\mathrm{ad}}=\left(\frac{\partial \ln \mathrm{T}}{\partial \ln \mathrm{P}}\right)_{\mathrm{S}} \quad(\text { gradiente de temperatura adiabático })
\end{gathered}
$$


onde os $\delta$ 's designam variações de grandezas físicas para uma mesma parcela de fluido, isto é, variações Lagrangeanas.

As relações que definem $\varepsilon_{\mathrm{n}}$ e $\mathrm{k}$ dependem também da composição química e serão consideradas quando necessário.

As evidências observacionais sugerem que as PNR causam efeitos muito pequenos comparados à estrutura de equilíbrio das estrelas. Isto simplifica enormemente a solução das equações hidrodinâmicas acima permitindo descrever as oscilações como perturbações sobrepostas a um estado de equilíbrio estático da estrela.

\subsubsection{Estrela em equilíbrio estático}

Assumindo uma situação de equilíbrio estático e as hipóteses 1) a 5), temos $\overrightarrow{\mathrm{u}}=\overrightarrow{\mathrm{u}}_{0}=0, \delta \mathrm{S}=0$ nulos, e as grandezas físicas dependerão somente do raio $\mathrm{r}$ (simetria esférica); isto nos leva às conhecidas equações de estrutura estelar obtidas a partir das equações básicas acima ${ }^{4}$ :

$$
\begin{gathered}
(3.4) \Rightarrow \quad \text { satisfeita } \\
(3.5) \Rightarrow \quad \frac{\mathrm{dP}_{0}}{\mathrm{dr}}=-\rho_{0} \frac{\mathrm{d} \phi_{0}}{\mathrm{dr}} \equiv-\rho_{0} \mathrm{~g}_{0} \quad \text { (Equilíbrio hidrostático) } \\
(3.6) \Rightarrow \quad \rho_{0} \varepsilon_{\mathrm{n} .0}=\frac{1}{\mathrm{r}^{2}} \frac{\mathrm{d}}{\mathrm{dr}}\left(\mathrm{r}^{2} \mathrm{~F}_{\mathrm{Rad}, 0}\right)=\frac{1}{4 \pi \mathrm{r}^{2}} \frac{\mathrm{dL}_{0}}{\mathrm{dr}} \quad \text { (Equilíbrio térmico) }
\end{gathered}
$$

onde $\mathrm{L}_{0}=4 \pi \mathrm{r}^{2} \mathrm{~F}_{\mathrm{Rad}, 0}$ é a luminosidade que atravessa uma esfera de raio $\mathrm{r}$. Na verdade a estrela pode não estar totalmente no equilíbrio térmico pois o tempo para a mesma se ajustar à condição fora do equilíbrio ( tempo de Kelvin-Helmholtz) é muito maior do que o período das oscilações.

$$
\text { (3.7) } \Rightarrow \quad g_{0} \equiv \frac{d \phi_{0}}{d r}=\frac{\mathrm{GM}_{\mathrm{r}}}{\mathrm{r}^{2}}
$$

onde $\mathrm{M}_{\mathrm{r}} \equiv 4 \pi \mathrm{G} \int_{0}^{\mathrm{r}} \rho_{0}(\mathrm{r}) \mathrm{r}^{2} \mathrm{dr}$.

\footnotetext{
${ }^{4}$ o subscrito 0 representa a condição de equilíbrio.
} 


$$
\text { (3.8) } \Rightarrow \quad \mathrm{F}_{\mathrm{Rad}, 0}=-\left(\frac{4 \mathrm{ac}}{3 \mathrm{k}_{0}} \mathrm{~T}_{0}^{3}\right) \frac{\mathrm{dT}_{0}}{\mathrm{dr}} \equiv-\mathrm{K}_{0} \frac{\mathrm{dT}_{0}}{\mathrm{dr}}
$$

\subsubsection{Equações das oscilações (análise de perturbação)}

Vamos analisar o efeito de pequenas perturbações sobre o estado de equilíbrio descrito acima. As perturbações sobre uma grandeza física $\mathrm{f}$ qualquer podem ser representadas de duas formas diferentes:

1) Perturbação Euleriana (') - perturbação da quantidade física numa dada posição fixa.

$$
f(\vec{r}, t)=f_{0}(r)+f^{\prime}(\vec{r}, t)
$$

2) Perturbação Lagrangeana $(\delta)$ - perturbação da quantidade física associada a um dado elemento de massa do fluido.

$$
f(\vec{r}, t)=f_{0}(r)+\delta f(\vec{r}, t)
$$

As perturbações Lagrangeana e Euleriana relacionam-se entre si através da transformação:

$$
\delta \mathrm{f}(\overrightarrow{\mathrm{r}}, \mathrm{t})=\mathrm{f}^{\prime}(\overrightarrow{\mathrm{r}}, \mathrm{t})+\vec{\xi}(\overrightarrow{\mathrm{r}}, \mathrm{t}) \cdot \vec{\nabla} \mathrm{f}_{0}(\mathrm{r})
$$

onde $\vec{\xi}=\left(\xi_{\mathrm{r}}, \xi_{\theta}, \xi_{\varphi}\right) \equiv \overrightarrow{\mathrm{r}}-\overrightarrow{\mathrm{r}}_{0}$ é o vetor deslocamento de um elemento de fluido que no equilíbrio encontrava-se em $\vec{r}_{0}$ e com a perturbação deslocou-se para $\overrightarrow{\mathrm{r}}$. Para aplicarmos a teoria linear de perturbações é necessário que $|\delta \mathrm{f}|<<\mathrm{f}_{0}\left(\left|\mathrm{f}^{\prime}\right|<<\mathrm{f}_{0}\right)$.

Vamos trabalhar com o campo de deslocamento ao invés das velocidades perturbadas; a derivada Lagrangeana do deslocamento de um elemento de fluido é a perturbação Lagrangeana da velocidade, ou seja,

$$
\delta \overrightarrow{\mathrm{v}}=\frac{\mathrm{d} \vec{\xi}}{\mathrm{dt}}=\frac{\partial \vec{\xi}}{\partial \mathrm{t}}+(\overrightarrow{\mathrm{v}} \cdot \vec{\nabla}) \vec{\xi} \cong \frac{\partial \vec{\xi}}{\partial \mathrm{t}}+\left(\overrightarrow{\mathrm{v}}_{0} \cdot \vec{\nabla}\right) \vec{\xi}
$$

Utilizando (3.18) temos:

$$
\overrightarrow{\mathrm{v}}^{\prime}=\delta \overrightarrow{\mathrm{v}}-(\vec{\xi} \cdot \vec{\nabla}) \overrightarrow{\mathrm{v}}_{0}=\frac{\partial \vec{\xi}}{\partial \mathrm{t}}+\left(\overrightarrow{\mathrm{v}}_{0} \cdot \vec{\nabla}\right) \vec{\xi}-(\vec{\xi} \cdot \vec{\nabla}) \overrightarrow{\mathrm{v}}_{0}
$$

Entretanto, conforme já havíamos dito, $\overrightarrow{\mathrm{v}}_{0}=0$ na ausência de rotação $\mathrm{e}$ convecção e a equação acima torna-se simplesmente 


$$
\overrightarrow{\mathrm{v}}^{\prime}=\delta \overrightarrow{\mathrm{v}}=\frac{\partial \vec{\xi}}{\partial \mathrm{t}}=\frac{\mathrm{d} \vec{\xi}}{\mathrm{dt}}
$$

Finalmente, as equações linearizadas são obtidas substituindo a (3.16) nas equações básicas (3.4) a (3.8) e eliminando os termos de $2^{\mathrm{a}}$ ordem ou maior nas perturbações, assim como os termos correspondentes ao estado de equilíbrio dado pelas relações (3.12) a (3.15):

$$
\begin{gathered}
(3.4) \Rightarrow \frac{\partial \rho^{\prime}}{\partial \mathrm{t}}+\vec{\nabla} \cdot\left(\rho \frac{\partial \vec{\xi}}{\partial \mathrm{t}}\right)=0 \\
(3.5) \Rightarrow \rho \frac{\partial}{\partial \mathrm{t}}\left(\frac{\partial \vec{\xi}}{\partial \mathrm{t}}\right)=-\rho^{\prime} \frac{\mathrm{d} \phi}{\mathrm{dr}} \hat{\mathrm{r}}-\vec{\nabla} \mathrm{P}^{\prime}-\rho \vec{\nabla} \phi^{\prime}
\end{gathered}
$$

$\mathrm{Na}$ equação do movimento perturbada acima existem 3 termos de força restauradoras, sendo que os dois primeiros representam as forças devidos ao empuxo ("buoyancy") e às variações de pressão respectivamente, enquanto que o último leva em conta a variação do potencial gravitacional e em geral pode ser desprezado (aproximação de Cowling). É útil separarmos a parte radial ( $\hat{\mathrm{r}})$ da horizontal ( $\hat{\theta}$ e $\hat{\varphi})$ na (3.23):

$$
\begin{aligned}
& (\hat{\mathrm{r}}) \quad \Rightarrow \quad \frac{\partial^{2} \xi_{\mathrm{r}}}{\partial \mathrm{t}^{2}}=-\frac{\rho^{\prime}}{\rho} \frac{\mathrm{d} \phi}{\mathrm{dr}}-\frac{1}{\rho} \frac{\partial \mathrm{P}^{\prime}}{\partial \mathrm{r}}-\frac{\partial \phi^{\prime}}{\partial \mathrm{r}} \\
& (\hat{\theta} \text { e } \hat{\varphi}) \Rightarrow \quad \frac{\partial^{2} \vec{\xi}_{\perp}}{\partial \mathrm{t}^{2}}=-\vec{\nabla}_{\perp}\left(\frac{\mathrm{P}^{\prime}}{\rho}+\phi^{\prime}\right) \\
& \text { onde } \vec{\nabla}_{\perp}=\frac{1}{\mathrm{r}}\left(0, \frac{\partial}{\partial \theta}, \frac{1}{\operatorname{sen} \theta} \frac{\partial}{\partial \varphi}\right) \text {. } \\
& \text { (3.6) } \Rightarrow \quad \rho \mathrm{T} \frac{\partial}{\partial \mathrm{t}}\left(\mathrm{S}^{\prime}+\vec{\xi} \cdot \vec{\nabla} \mathrm{S}_{0}\right)=\rho \mathrm{T} \frac{\partial \delta \mathrm{S}}{\partial \mathrm{t}}=\left(\rho \varepsilon_{\mathrm{n}}\right)^{\prime}-\vec{\nabla} \cdot \overrightarrow{\mathrm{F}}^{\prime} \\
& \text { (3.7) } \Rightarrow \quad \vec{\nabla}^{2} \phi^{\prime}=4 \pi \mathrm{G} \rho^{\prime} \\
& \text { (3.8) } \Rightarrow \quad \overrightarrow{\mathrm{F}}^{\prime}=\overrightarrow{\mathrm{F}}_{\mathrm{Rad}}^{\prime}=-\mathrm{K}^{\prime} \frac{\mathrm{dT}}{\mathrm{dr}}-\mathrm{K} \vec{\nabla} \mathrm{T}^{\prime}
\end{aligned}
$$


$\mathrm{O}$ índice 0 foi removido das grandezas correspondentes ao equilíbrio para simplificar a notação. As variações Eulerianas são funções de $(\vec{r}, t)$, enquanto as quantidades físicas no equilíbrio dependem apenas de $\mathrm{r}$.

Podemos aplicar o método de separação de variáveis ao sistema perturbado (3.24) a (3.28), separando as dependências radiais, angulares e temporais. Não serão mostradas aqui todas as passagens deste procedimento, apenas indicados os passos principais que levam às equações finais:

- eliminar a variável $\vec{\xi}_{\perp}$ através da equação (3.25);

- eliminar $\rho$ ' através de (3.9) e (3.18);

- supor uma separação de variáveis do tipo

$$
f^{\prime}(\vec{r}, t)=f^{\prime}(r) Y(\theta, \varphi) e^{\text {iot }}
$$

onde $\mathrm{Y}$ é uma função qualquer e $\omega$ é uma constante complexa. A variável $\delta \mathrm{S}$ é escrita como (3.29), porém mantém sua forma Lagrangeana. Será utilizado o mesmo símbolo para $\mathrm{f}^{\prime}(\overrightarrow{\mathrm{r}}, \mathrm{t})$ e $\mathrm{f}^{\prime}(\mathrm{r})$;

Como os coeficientes das equações perturbadas são funções apenas de r, a parte angular das variáveis somente sofre a ação das derivadas angulares do operador Laplaciano. Assim, para que a parte angular seja separada da parte radial é necessário que

$$
\vec{\nabla}_{\perp}^{2} Y(\theta, \varphi)=\frac{1}{r^{2} \operatorname{sen}^{2} \theta}\left[\operatorname{sen} \theta \frac{\partial}{\partial \theta}\left(\operatorname{sen} \theta \frac{\partial}{\partial \theta}\right)+\frac{\partial^{2}}{\partial \varphi^{2}}\right] Y(\theta, \varphi)=-\frac{\Lambda}{r^{2}} Y(\theta, \varphi)
$$

onde $\Lambda$ é uma constante complexa. Levando-se em conta a periodicidade angular de $2 \pi$ rad, a regularidade e os limites que Y deve satisfazer, as soluções da (3.30) devem ser funções harmônicos esféricos:

$$
Y_{\ell}^{m}(\theta, \varphi)=N_{\ell}^{m} P_{\ell}^{|m|}(\cos \theta) e^{i m \varphi}
$$

Portanto a parte angular pode ser separada facilmente uma vez que os harmônicos esféricos respeitam a equação,

$$
\vec{\nabla}_{\perp}^{2} Y_{\ell}^{m}(\theta, \varphi)=-\frac{\ell(\ell+1)}{r^{2}} Y_{\ell}^{m}(\theta, \varphi)
$$

onde

$\ell \geq 0$ (inteiro) $\Rightarrow \quad$ grau (onde $\Lambda=\ell(\ell+1)$ );

$|\mathrm{m}| \leq \ell \quad \Rightarrow \quad$ ordem azimutal;

$P_{\ell}^{|m|}(\cos \theta)$ são os polinômios associados de Legendre do $1^{\circ}$ tipo;

$N_{\ell}^{m}$ é uma constante de normalização. 


\subsubsection{Equações básicas das PNR lineares e não-adiabáticas}

Deste modo as equações perturbadas para a dependência radial $\left(\mathrm{f}^{\prime}(\mathrm{r})\right)$ podem ser escritas como

Equação da continuidade:

$$
\text { (3.22) } \Rightarrow \frac{1}{r^{2}} \frac{d\left(r^{2} \xi_{r}\right)}{d r}+\frac{1}{\Gamma_{1}} \frac{d \ln P}{d r} \xi_{r}+\left(1-\frac{L_{\ell}^{2}}{\omega^{2}}\right) \frac{P^{\prime}}{\rho v_{s}^{2}}-\frac{\ell(\ell+1)}{r^{2} \omega^{2}} \phi^{\prime}=\nabla_{a d} \frac{\rho T}{P} \delta S
$$

Parte radial da equação do movimento:

$$
(3.24) \Rightarrow \frac{1}{\rho} \frac{d P^{\prime}}{d r}+\frac{g}{\rho v_{s}^{2}} P^{\prime}+\left(N^{2}-\omega^{2}\right) \xi_{r}+\frac{d \phi^{\prime}}{d r}=g \nabla_{a d} \frac{\rho T}{P} \delta S
$$

Conservação da energia:

$$
(3.26) \Rightarrow\left(\rho \varepsilon_{n}\right)^{\prime}-\frac{1}{r^{2}} \frac{d\left(r^{2} F_{r}^{\prime}\right)}{d r}-\frac{\ell(\ell+1)}{r^{2}} K T^{\prime}=i \omega \rho T \delta S
$$

Equação de Poisson:

$$
\text { (3.27) } \Rightarrow \frac{1}{r^{2}} \frac{d}{d r}\left(r^{2} \frac{d \phi^{\prime}}{d r}\right)-\frac{\ell(\ell+1)}{r^{2}} \phi^{\prime}-4 \pi G \rho\left(\frac{P^{\prime}}{\rho v_{s}^{2}}+\frac{N^{2} \xi_{r}}{g}\right)=-4 \pi G \nabla_{a d} \frac{\rho^{2} T}{P} \delta S
$$

Componente radial do fluxo radiativo:

$$
\text { (3.28) } \Rightarrow \mathrm{F}_{\mathrm{r}}{ }^{\prime}=-\mathrm{K} \frac{\mathrm{dT}^{\prime}}{\mathrm{dr}}-\mathrm{K}^{\prime} \frac{\mathrm{dT}}{\mathrm{dr}}
$$

onde $\mathrm{v}_{\mathrm{s}}=\sqrt{\frac{\Gamma_{1} \mathrm{P}}{\rho}}$ é a velocidade do som. Os termos $\mathrm{N}^{2}$ e $L_{\ell}^{2}$ são duas frequências características, a freqüência de Brunt-Väissälä e a freqüência de Lamb. A freqüência de Brunt-Väissälä é dada por:

$$
\mathrm{N}^{2}=\mathrm{N}^{2}(\mathrm{r})=-\mathrm{gA}=\mathrm{g}\left(\frac{1}{\Gamma_{1}} \frac{\mathrm{d} \ln \mathrm{P}}{\mathrm{dr}}-\frac{\mathrm{d} \ln \rho}{\mathrm{dr}}\right)
$$

onde A indica o grau de instabilidade convectiva, de modo que

$\mathrm{A}>0\left(\mathrm{~N}^{2}<0\right) \Rightarrow$ instabilidade convectiva;

e

$\mathrm{A}<0\left(\mathrm{~N}^{2}>0\right) \Rightarrow$ estabilidade (ausência de convecção). 
$\mathrm{N}^{2}$ pode ser reescrito numa outra forma que explicita a dependência dos gradientes de pressão de densidade com a composição química. Assim, supondo que a pressão na região totalmente ionizada do interior estelar é dada pela equação do gás ideal, $\mathrm{N}^{2}$ torna-se

$$
\mathrm{N}^{2} \cong \frac{\mathrm{g}^{2} \rho}{\mathrm{P}}\left(\nabla_{\mathrm{ad}}-\nabla+\nabla_{\mu}\right)
$$

onde $\nabla=\frac{\mathrm{d} \ln \mathrm{T}}{\mathrm{d} \ln \mathrm{P}}, \nabla_{\mathrm{ad}}=\left(\frac{\partial \ln \mathrm{T}}{\partial \ln \mathrm{P}}\right)_{\mathrm{ad}}, \nabla_{\mu}=\frac{\mathrm{d} \ln \mu}{\mathrm{d} \ln \mathrm{P}}$ e $\mu$ é o peso molecular médio. Nesta forma é fácil ver que regiões convectivas $\left(\nabla>\nabla_{\mathrm{ad}}\right.$ ) implicam em $\mathrm{N}^{2}<0$ (supondo $\nabla \mu \cong$ 0 ), mas apesar de ser negativo a frequência de Brunt-Väisälä é pequena em módulo em estrelas com núcleos convectivos. Na região onde há queima nuclear, $\mu$ aumenta com a profundidade, isto é, com a pressão, e o termo $\nabla_{\mu}$ contribui aumentando o valor de $\mathrm{N}^{2}$.

$\mathrm{N}$ é a frequência com a qual uma parcela de fluido perturbada oscila num meio convectivamente estável tendo a gravidade como força restauradora. A outra frequência importante é a freqüência de Lamb, dada por:

$$
L_{l}^{2}=L_{l}^{2}(r)=\frac{\ell(\ell+1)}{r^{2}} v_{s}^{2}
$$

Note que $2 \pi / L_{\ell}^{2}$ é o tempo que leva uma onda com $\mathrm{v}_{\text {fase }}=\mathrm{v}_{\mathrm{s}}$ para percorrer uma distância dada por

$$
\lambda_{t}^{2} \equiv\left(\frac{2 \pi r}{\ell(\ell+1)}\right)^{2}
$$

onde $\lambda_{t}$ é o comprimento de onda transversal.

Note que temos sete variáveis ( $\mathrm{P}^{\prime}, \mathrm{T}^{\prime}, \delta \mathrm{S}, \phi^{\prime}, \xi_{\mathrm{r}},\left(\rho \varepsilon_{\mathrm{n}}\right)^{\prime}$ e $\left.\mathrm{F}_{\mathrm{r}}{ }^{\prime}\right)$ e apenas cinco equações (3.33 a 3.37). As duas equações restantes são dadas por uma relação termodinâmica entre pressão temperatura e entropia, e pela variação da produção de energia nuclear que depende da metalicidade da estrela como já mencionamos antes.

A solução deste sistema de equações diferenciais exige também condições de contorno apropriadas. A seguir apresentamos uma análise das oscilações adiabáticas onde as equações são grandemente simplificadas e as características principais das soluções podem ser estudadas mais facilmente. É útil descrever o vetor deslocamento como

$$
\vec{\xi}(\vec{r}, t)=\left(\xi_{r}(r), \xi_{t}(r) \frac{\partial}{\partial \theta}, \xi_{t}(r) \frac{1}{\sin \theta} \frac{\partial}{\partial \varphi}\right) Y_{\ell}^{m}(\theta, \varphi) e^{i \omega t}
$$

onde, pelas relações (3.25) e (3.29) temos: 


$$
\xi_{t}(r) \equiv \frac{1}{r \omega^{2}}\left(\frac{P^{\prime}(r)}{\rho(r)}+\phi^{\prime}(r)\right)
$$

\subsubsection{Modos toroidais}

Existe uma solução trivial das equações perturbadas (3.22) a (3.28) que é independente daquelas definidas por (3.31) e pelo sistema (3.33) a (3.37). Esta solução é obtida escolhendo-se todas as perturbações iguais a zero, exceto a velocidade que será do tipo $\vec{v}^{\prime}(\vec{r})=\left(0, v_{\theta}{ }^{\prime}, v_{\varphi}{ }^{\prime}\right)$. Assim, pela (3.22):

$$
\vec{\nabla} \cdot\left(\rho \frac{\partial \vec{\xi}}{\partial \mathrm{t}}\right)=\vec{\nabla} \cdot\left(\rho \overrightarrow{\mathrm{v}}^{\prime}\right)=0 \Rightarrow \vec{\nabla}_{\perp} \cdot \overrightarrow{\mathrm{v}}_{\perp}{ }^{\prime}=0
$$

Segundo a equação acima $\vec{v}^{\prime}$ é dado pelo rotacional de um campo vetorial $(\mathrm{V}(\overrightarrow{\mathrm{r}}) \hat{\mathrm{r}})$ que decomposto numa base de harmônicos esféricos torna-se

$$
\vec{v}^{\prime}(\vec{r})=\sum_{l, m} \frac{V_{\ell}^{m}(r)}{r}\left(0, \frac{1}{\sin \theta} \frac{\partial}{\partial \varphi},-\frac{\partial}{\partial \theta}\right) Y_{\ell}^{m}(\theta, \varphi)
$$

Estes são os modos toroidais e caracterizam-se por movimentos horizontais do tipo redemoinho estacionário. Por serem campos de velocidade não oscilatórios no tempo os modos toroidais não são interessantes para estrelas sem rotação. Entretanto, na presença de rotação e/ou campos magnéticos estes modos podem tornar-se importantes, gerando os chamados modos $\mathrm{r}$ (modos de Rossby).

\subsubsection{Características das PNR adiabáticas}

Para as estrelas em geral a escala de tempo dinâmica $\left(\mathrm{t}_{\mathrm{din}}\right)$ é pequena comparadas com a térmica ou de Kelvin-Helmholtz $\left(t_{\mathrm{KH}}\right)$, ou seja, os ajustes mecânicos são mais rápidos do que os térmicos. Para a estrela como um todo estas escalas de tempo são dadas por

$$
\begin{aligned}
& \mathrm{t}_{\mathrm{din}} \approx \sqrt{\frac{\mathrm{R}^{3}}{\mathrm{GM}}} \approx \text { horas } \\
& \mathrm{t}_{\mathrm{KH}} \approx \frac{\mathrm{GM}^{2}}{\mathrm{LR}} \approx \text { anos }
\end{aligned}
$$


onde os termos têm seus significados usuais da estrutura estelar. Este é o argumento que justifica a aproximação adiabática, onde as oscilações são estudadas supondo a ausência de trocas de calor durante as mesmas, ou seja, supondo $\delta \mathrm{S}=0$.

Vale lembrar que esta aproximação deixa de ser válida para as regiões externas da estrela onde $t_{\text {din }} \sim t_{\mathrm{KH}}$, bem como em algumas regiões internas onde ocorrem a excitação ou amortecimento das oscilações. Nestas regiões pode-se utilizar a aproximação quasi-adiabática em alguns casos.

A introdução da aproximação adiabática nas equações básicas (3.33) a (3.37) resulta num sistema muito mais simples dado apenas pelas (3.33), (3.34) e (3.36), sem os termos proporcionais a $\delta \mathrm{S}$. As outras equações não são necessárias uma vez que as trocas de calor estão sendo desprezadas. Temos então as chamadas equações básicas das PNR lineares e adiabáticas

$$
\begin{gathered}
\text { (3.33) } \Rightarrow \frac{1}{r^{2}} \frac{d\left(r^{2} \xi_{r}\right)}{d r}+\frac{1}{\Gamma_{1}} \frac{d \ln P}{d r} \xi_{r}+\left(1-\frac{L_{\ell}^{2}}{\omega^{2}}\right) \frac{P^{\prime}}{\rho v_{s}^{2}}-\frac{\ell(\ell+1)}{r^{2} \omega^{2}} \phi^{\prime}=0 \\
\text { (3.34) } \Rightarrow \frac{1}{\rho} \frac{\mathrm{dP}}{\mathrm{dr}}+\frac{\mathrm{g}}{\rho \mathrm{v}_{\mathrm{s}}^{2}} \mathrm{P}^{\prime}+\left(\mathrm{N}^{2}-\omega^{2}\right) \xi_{\mathrm{r}}+\frac{\mathrm{d} \phi^{\prime}}{\mathrm{dr}}=0 \\
(3.36) \Rightarrow \frac{1}{r^{2}} \frac{d}{d r}\left(r^{2} \frac{d \phi^{\prime}}{d r}\right)-\frac{\ell(\ell+1)}{r^{2}} \phi^{\prime}-4 \pi G \rho\left(\frac{P^{\prime}}{\rho v_{s}^{2}}+\frac{N^{2} \xi_{r}}{g}\right)=0
\end{gathered}
$$

Este sistema é bem mais simples que o anterior, podendo ser reduzido a um sistema de 4 equações diferenciais lineares de $1^{\text {a }}$ ordem com coeficientes reais dados pela estrutura de equilíbrio e com 4 variáveis adimensionais escolhidas apropriadamente (Kippenhahn \& Weigert, 1994). São necessárias portanto 4 condições de contorno para o sistema acima, sendo duas em $r=0$ e duas em $r=R$ :

$$
\begin{aligned}
& \frac{d \phi^{\prime}}{d r}-\frac{\ell \phi^{\prime}}{r}=0 \\
& \underline{\mathrm{r}=0} \quad \Rightarrow \\
& \xi_{r}=\frac{\ell}{r \omega^{2}}\left(\frac{P^{\prime}}{\rho}+\phi^{\prime}\right)=\ell \xi_{t}
\end{aligned}
$$

e 


$$
\begin{gathered}
\frac{d \phi^{\prime}}{d r}+\frac{(\ell+1) \phi^{\prime}}{r}=0 \\
\delta \mathrm{P}=0 \Rightarrow \vec{\nabla} \cdot \vec{\xi}=0 \Rightarrow \xi_{\mathrm{r}}=\frac{\omega^{2}}{\left(\frac{\mathrm{GM}}{\mathrm{R}^{3}}\right)} \xi_{\mathrm{t}} \equiv \sigma^{2} \xi_{\mathrm{t}}
\end{gathered}
$$

A escolha das condições de contorno não é uma tarefa simples, principalmente na superfície e em geral os autores discutem outras possibilidades para as mesmas. Na equação (3.54) utilizamos a aproximação de Cowling onde $\phi^{\prime}=0$. Além disso $\sigma^{2}$ é chamada de frequência adimensional (adotando a mesma notação utilizada por Christensen-Dalsgaard, 1989 e Kippenhahn \& Weigert 1994).

As equações (3.48) a (3.50) e as condições de contorno (3.51) a (3.54) formam um problema de autovalores com autovalor dado por $\omega^{2}$. Como a ordem azimutal $m$ não aparece nas equações e nas condições de contorno os autovalores são degenerados em m, isto é, para cada autovalor $\omega^{2}$ existem $(2 \ell+1)$ autofunções do tipo (3.29); esta degenerescência é removida na presença de rotação e/ou campo magnético

Fixando $\ell$ nas equações (3.48) a (3.50) e resolvendo as dependências radiais $\left(\mathrm{P}^{\prime}, \phi ', \xi_{\mathrm{r}}\right)$ teremos um conjunto infinito de soluções que em geral são identificadas por um inteiro $\mathrm{n}$ (ordem radial). Vemos portanto que os autovalores $\omega^{2}$ dependem de $\ell$ e $n$ sendo normalmente escritos como $\omega_{\ell, n}^{2}$. Nos casos simples n é o número de zeros para as dependências radiais; por exemplo

$$
\xi_{\mathrm{r}}\left(\mathrm{r}_{\mathrm{i}}\right)=0 ; \quad(\mathrm{i}=1,2, \ldots, \mathrm{n}) \text { e } \mathrm{r} \neq 0
$$

Pode ser mostrado (Unno et al., 1989) que este problema de autovalores é autoadjunto (ou hermitiano) apesar de não ser do tipo Sturm-Liouville, pois existem duas forças restauradoras diferentes. Isto garante que os autovalores são reais de modo que as oscilações são puramente periódicas $\left(\omega_{\ell, n}^{2}>0\right.$; estabilidade dinâmica) ou puramente aperiódicas $\left(\omega_{\ell, n}^{2}<0\right.$; instabilidade dinâmica). Outra consequência é a ortogonalidade das autofunções, desde que não exista degenerescência dos autovalores em relação à ordem radial $\mathrm{n}$.

Como estamos tratando de operadores lineares, podemos descrever uma perturbação f' (= P', $\phi$ ', ou $\xi_{\mathrm{r}}$ ) qualquer como uma combinação linear das autofunções,

$$
f^{\prime}(\vec{r}, t)=\sum_{\ell, m, n} f_{\ell, n}(r) Y_{\ell}^{m}(\theta, \varphi) \exp \left(i \omega_{\ell, n} t\right)
$$

onde

$$
\begin{array}{ll}
\mathrm{n} \geq 0 \text { (inteiro) } \Rightarrow & \text { ordem radial; } \\
\ell \geq 0 \text { (inteiro) } \Rightarrow & \text { grau; } \\
|\mathrm{m}| \leq \ell & \Rightarrow \quad \text { ordem azimutal; }
\end{array}
$$


$f_{\ell, n}(r)$ é dada pelas equações (3.48) a (3.54) $\left(P_{\ell, n}, \phi_{\ell, n}\right.$, ou $\left.\xi_{r, \ell, n}\right)$;

$\omega_{\ell, n}$ é a raiz positiva do autovalor para $\ell$ e $\mathrm{n}$ fixos;

$Y_{\ell}^{m}(\theta, \varphi)$ são os harmônicos esféricos definidos em (3.31).

A equação (3.56) permite interpretarmos as perturbações como sendo formadas por ondas estacionárias $\left(\mathrm{f}^{\prime} \propto \exp (\mathrm{im} \varphi) \exp (\mathrm{i} \omega \mathrm{t})\right)$ como também por ondas propagando-se $\left(\mathrm{f}^{\prime} \propto \exp (\mathrm{i}(\mathrm{m} \varphi+\omega \mathrm{t}))\right.$ com velocidade de fase

$$
\frac{\mathrm{d} \varphi}{\mathrm{dt}}=-\frac{\omega}{\mathrm{m}}
$$

Entretanto, quando existe rotação e/ou campo magnético, apenas as ondas em propagação podem representar modos normais de oscilação pois as frequências também dependerão de $\mathrm{m}\left(\omega_{\ell, m, n}\right)$. Neste caso $\mathrm{m}>0$ representa modos retrógrados e $\mathrm{m}<0$ modos prógrados em relação à rotação da estrela.

\subsubsection{Análise local das soluções}

Em geral não é possível aplicar um tratamento analítico ao sistema linear e adiabático definido por (3.48) a (3.50), porém algumas simplificações podem ser adotadas possibilitando uma discussão analítica das soluções.

Uma grande simplificação é obtida com a aproximação de Cowling, na qual a perturbação no potencial gravitacional é desprezada $\left(\phi^{\prime}=0\right)$. Isto reduz pela metade a ordem do sistema (3.48) a (3.50), o qual passa então a ser descrito por duas equações diferenciais lineares de $1^{\mathrm{a}}$ ordem e duas condições de contorno (3.52 e 3.54). Note que mesmo na aproximação de Cowling o problema não é do tipo Sturm-Liouville, uma vez que ainda existem duas forças restauradoras fazendo com que os autovalores $\omega_{\ell, n}^{2}$ figurem tanto no numerador como no denominador.

Podemos ter uma indicação da validade da aproximação de Cowling através da solução formal para a dependência radial da equação de Poisson perturbada (3.27):

$$
\phi^{\prime}(r)=\frac{4 \pi G}{2 \ell+1}\left[\frac{1}{r^{l+1}} \int_{0}^{r} \rho^{\prime}(\widetilde{r}) \widetilde{r}^{l+2} d \widetilde{r}+r^{l} \int_{r}^{R} \frac{\rho^{\prime}(\widetilde{r})}{\widetilde{r}^{l-1}} d \widetilde{r}\right]
$$

Esta equação mostra que $\left|\phi^{\prime}\right|<<\rho$ ', ou seja, que a aproximação de Cowling é válida quando $\ell$ e/ou n é grande. Alguns trabalhos com simulações numéricas, onde não há necessidade de adotar-se $\phi^{\prime}=0$, mostraram que esta aproximação é realmente válida para modos com $\ell$ e/ou n elevados. As equações (3.48) a (3.50) simplificam-se então para

$$
\text { (3.48) } \Rightarrow \frac{d \xi_{r}}{d r}=-\left(\frac{2}{r}+\frac{1}{\Gamma_{1}} \frac{d \ln P}{d r}\right) \xi_{r}+\frac{1}{\rho v_{s}^{2}}\left(\frac{L_{\ell}^{2}}{\omega^{2}}-1\right) P^{\prime}
$$




$$
\begin{aligned}
& (3.49) \Rightarrow \frac{\mathrm{dP}^{\prime}}{\mathrm{dr}}=\rho\left(\omega^{2}-\mathrm{N}^{2}\right) \xi_{\mathrm{r}}+\frac{1}{\Gamma_{1}} \frac{\mathrm{d} \ln \mathrm{P}}{\mathrm{dr}} \mathrm{P}^{\prime} \\
& (3.50) \Rightarrow \text { Não é necessária na aproximação de Cowling }
\end{aligned}
$$

onde a (3.12) também foi utilizada para obter a (3.59). Note que a escala de altura de pressão é dada por $\mathrm{H}_{\mathrm{P}}=-\left(\frac{\mathrm{d} \ln \mathrm{P}}{\mathrm{dr}}\right)^{-1}$, que é aproximadamente a distância ao longo da qual a pressão diminui por um fator $1 / \mathrm{e}$.

Vamos agora, consistentemente com a aproximação de Cowling, ignorar o primeiro termo à direita de (3.59) e o último termo de (3.60); além disso vamos tratar como constantes todas as grandezas, exceto $\xi_{\mathrm{r}}$ e P'. Assim, isolando P' em (3.59) e substituindo em (3.60) temos a equação mais simplificada possível para as oscilações não-radiais:

$$
\frac{d^{2} \xi_{r}}{d r^{2}}=-\frac{1}{\omega^{2} v_{s}^{2}}\left(\omega^{2}-L_{\ell}^{2}\right)\left(\omega^{2}-N^{2}\right)_{r} \equiv-k_{r}^{2} \xi_{r}
$$

O estudo da equação acima constitui uma análise local das oscilações e, apesar de sua dedução conter muitos passos questionáveis, ela descreve adequadamente as propriedades gerais dos modos de oscilação.

Na verdade $\mathrm{k}_{\mathrm{r}}$, o número de onda radial, varia com $\mathrm{r}$, mas na análise local ele é constante numa região pequena de alguns comprimentos de onda; lembre-se que $k_{\mathrm{r}}$ é grande pois n é grande na aproximação de Cowling. A solução de (3.61) para $\mathrm{k}_{\mathrm{r}}$ constante é do tipo $\xi_{\mathrm{r}} \propto \exp \left( \pm \mathrm{ik_{ \textrm {r } }} \mathrm{r}\right)$, resultando na seguinte relação:

$$
k_{r}^{2}=\frac{1}{\omega^{2} v_{s}^{2}}\left(\omega^{2}-L_{\ell}^{2}\right)\left(\omega^{2}-N^{2}\right)
$$

Esta relação mostra dois comportamentos diferentes para a dependência radial das autofunções determinados pelas freqüências de Lamb e Brunt-Väisälä:

- $\omega^{2}<L_{\ell}^{2}, N^{2}$ ou $\omega^{2}>L_{\ell}^{2}, N^{2} \quad \Rightarrow \quad \mathrm{k}_{\mathrm{r}}^{2}>0$ (as soluções oscilam com $\mathrm{r}$ );

- $N^{2}<\omega^{2}<L_{\ell}^{2}$ ou $L_{\ell}^{2}<\omega^{2}<N^{2} \Rightarrow \mathrm{k}_{\mathrm{r}}^{2}<0$ (variação exponencial com $\mathrm{r}$ ).

Assim, para uma frequência fixa existem regiões no interior estelar onde as autofunções oscilam e regiões onde elas variam exponencialmente. As regiões de oscilação (zonas de propagação) estão separadas das regiões com comportamento exponencial (zonas evanescentes) pelas frequências críticas $\mathrm{N}^{2}$ e $L_{\ell}{ }^{2}$. Portanto as soluções oscilantes estão confinadas (trapped) nas zonas de propagação que são limitadas pelos pontos de retorno (turning points) onde $\mathrm{k}_{\mathrm{r}}=0$. 
Todos estes conceitos são visualizados de modo simples nos chamados diagramas de propagação, onde $\mathrm{N}^{2}$ e $L_{\ell}{ }^{2}$ são graficados em função de $\mathrm{r}$ e os autovalores $\omega$ são linhas horizontais que atravessam as zonas evanescentes e de propagação. Como $L_{\ell}$ depende do grau $\ell$ e $\mathrm{N}^{2}$ depende do gradiente do peso molecular médio, então os diagramas de propagação são construídos para $\ell$ e idade estelar fixos. 


\section{4- MÉTODOS DE ANÁLISE}

\subsection{Método dos Resíduos}

Esse método consiste no estudo das variações dos perfis de linha (com amplitudes de $\sim 1 \%$ em relação ao contínuo) a partir dos resíduos dos perfis espectrais (Yang et al., 1988). Os resíduos são formados a partir da diferença entre o espectro médio (suposto isento de tais variações, também conhecidas como bumps, pois elas devem se cancelar no tempo) e cada um dos espectros que compõem o conjunto observado. Dessa forma podese observar diretamente a passagem de bumps pelo perfil de linha e estudar sua propagação em função do tempo. Adotando-se necessariamente um modelo de pulsação onde o modo setorial seja dominante, então esse método permite a determinação direta da freqüência de pulsação $\sigma$ e de sua ordem m. A Figura 10 mostra uma série de espectros centrados em Hel $\lambda 667.8 \mathrm{~nm}$ da estrela $\eta$ Centauri e seus respectivos resíduos.
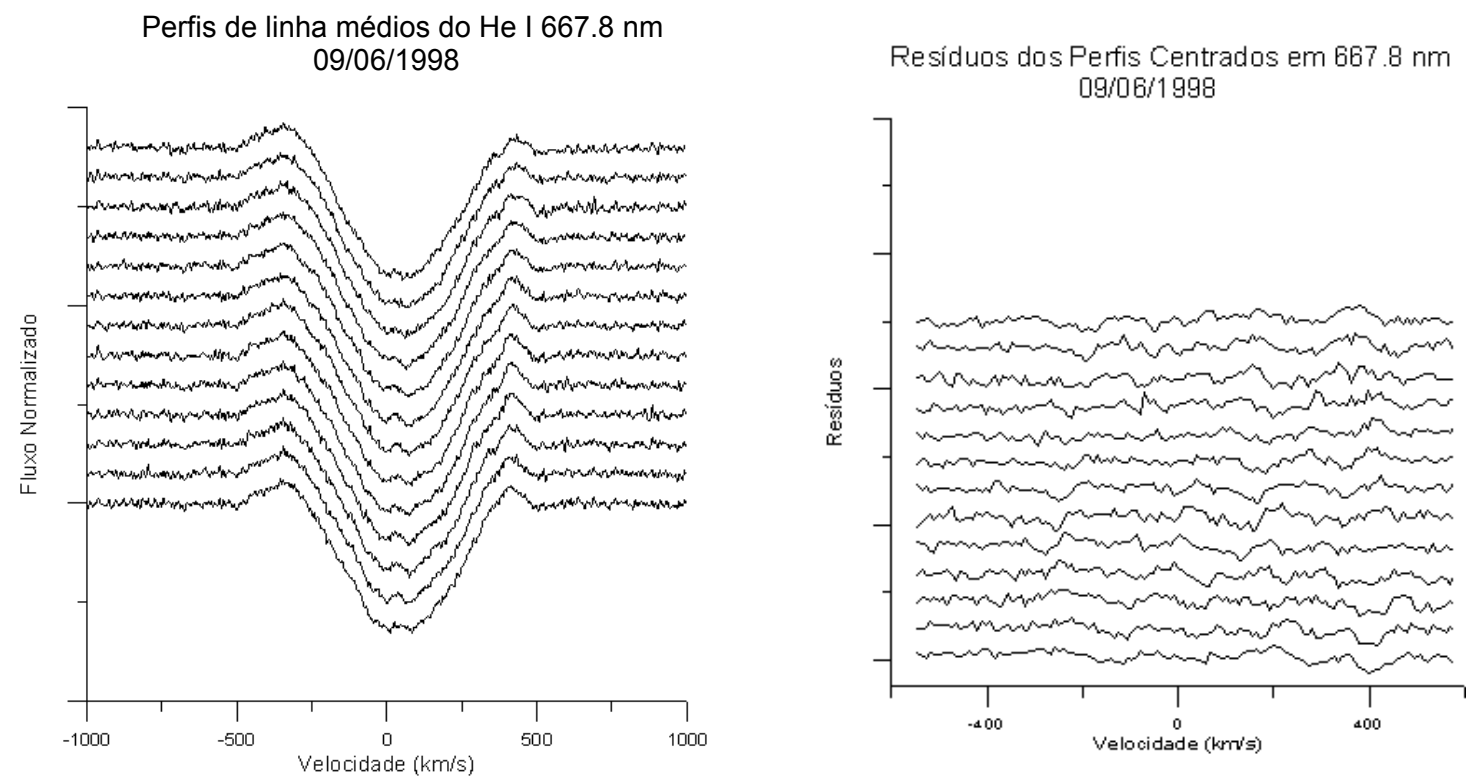

Fig.10. Espectros (à esquerda) e resíduos (à direita) da linha HeI $\lambda 667.8 \mathrm{~nm}$ da estrela $\eta$ Cen. Os espectros estão dispostos em ordem crescente no tempo de cima para baixo, enquanto os resíduos de baixo para cima.

Na Figura 11, vemos os mesmos resíduos mostrados na figura anterior, só que em escala de cinza obtido a partir de um aplicativo desenvolvido para ser usado com o pacote IDL. Os bumps podem ser vistos como manchas claras que caminham através do perfil de linha, do azul para o vermelho. 


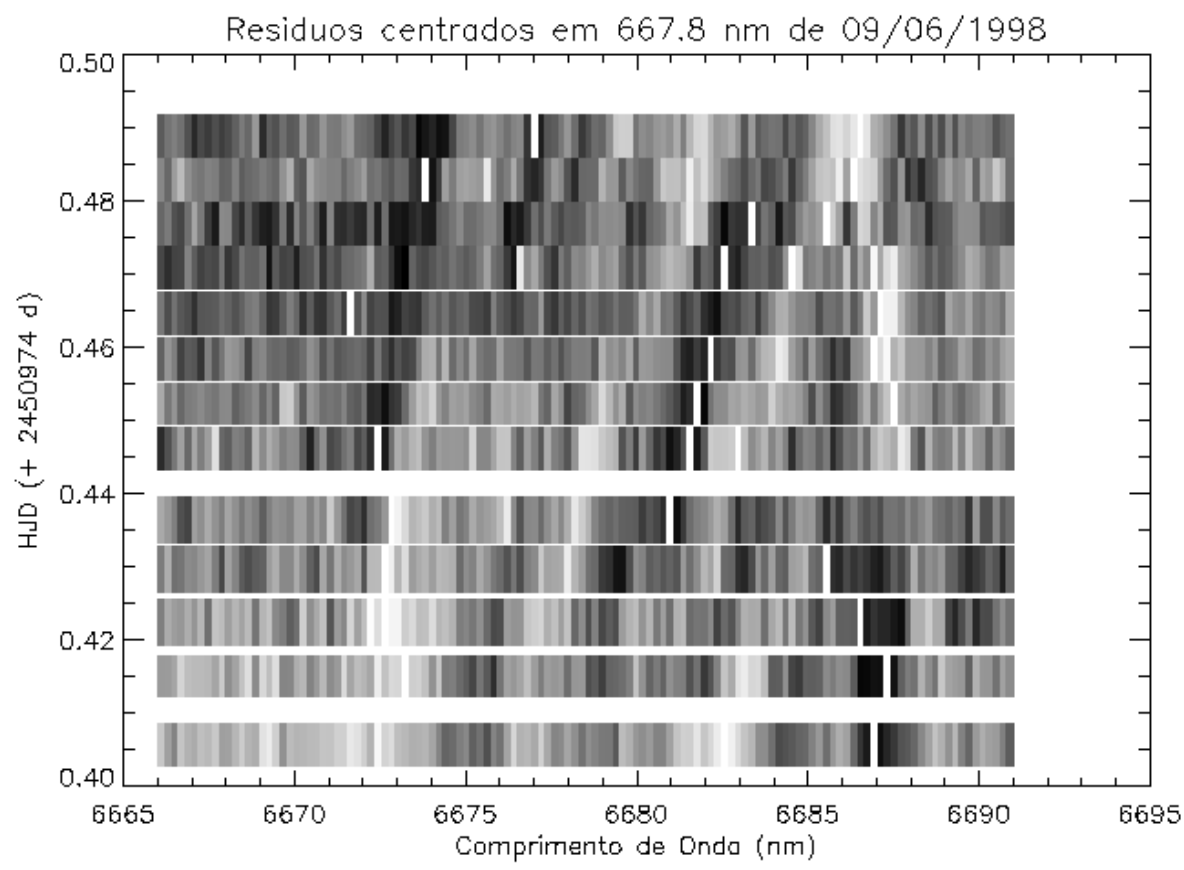

Fig.11. Resíduos dos espectros do dia 09/jun/1998 em escala de cinza.

Para a obtenção dos parâmetros pulsacionais, primeiramente os espectros devem ser graduados em velocidade $(\mathrm{km} / \mathrm{s})$, com o zero do eixo das velocidades localizado no centro da linha. Medem-se em seguida as posições dos bumps em velocidade para cada linha. Pode-se então construir um gráfico fornecendo a posição em velocidade dos mesmos em relação ao centro da linha em função do tempo e determinar sua aceleração.

Dois bumps que se movem através do perfil com a mesma aceleração são a manifestação de uma oscilação de mesmo modo. Medindo-se a diferença de tempo $\Delta \mathrm{t}$ que separam suas passagens pelo centro da linha, pode-se obter a freqüência $\sigma$ do seu modo associado:

$$
\sigma=\frac{1}{\Delta \mathrm{t}}
$$

Quando um modo de grau $m$ faz aparecer $2 m$ pontos nodais ao longo do equador estelar, existem $m$ bumps ao longo do equador. Como não é possível observar a metade de trás da estrela, então o número de bumps correspondentes ao mesmo modo que aparecem no perfil de uma linha é $m / 2$. Pode-se, então, encontrar $m$ através da fórmula (Hill et al., 1989):

$$
|m| \approx \frac{2 \pi\left(V_{e} \pm V_{\phi}\right) \sin i}{a_{0} \Delta t}
$$


onde $\mathrm{V}_{\mathrm{e}}$ é a velocidade de rotação equatorial da estrela, $\mathrm{V}_{\phi}$ é a velocidade de pulsação na direção azimutal e $a_{0}$ é a aceleração do bump. O sinal de + corresponde a um modo prógrado e o sinal de - a um modo retrógrado. No caso de estrelas Be com grandes velocidades de rotação, podemos negligenciar o termo $\mathrm{V}_{\phi}$ em (4.2) e $\sigma_{0}$ na fórmula $\sigma=\sigma_{0}-m \Omega$ para modos elevados, onde $\Omega$ é a freqüência de rotação da estrela. Então:

$$
|m|=\left|-\frac{\sigma}{\Omega}\right|
$$

Conhecendo-se $\sigma$, m e $\Omega$ de forma independente, pode-se então determinar $\sigma_{0}$ que é a freqüência do modo de pulsação no referencial da estrela. Este método permite identificar os diferentes modos de oscilação da estrela, entretanto na prática não funciona bem para modos baixos.

\subsection{Método da Transformada de Fourier}

Este método é mais poderoso que o método dos resíduos na determinação das freqüências. Parte-se, em princípio, de um conjunto de perfis de uma mesma linha espectral tomados em instantes diferentes de tempo. Constrói-se então, para cada comprimento de onda do perfil de linha, com passo $\Delta \lambda$, uma série temporal (tabela de intensidades relativas ao contínuo em função do tempo). Fazendo-se em seguida a transformada de Fourier dessas séries, obtém-se os espectros de potência com todas as freqüências da estrela convoluídas com a janela de observação (método de Gies \& Kullavanijaya, 1988). Além disso, o método de análise temporal fornece também informação sobre as fases das variações periódicas ao longo da linha espectral. Gies \& Kullavanijaya (1988) obtiveram uma medida de $|m|$ a partir da diferença das fases das variações temporais medidas nas bordas azul e vermelha da linha espectral.

Este trabalho não se dedica a discutir todos os métodos de análise temporal existentes na literatura. $\mathrm{O}$ objetivo consiste em se aplicar métodos razoáveis que possam dar condições de se obter periodicidades verdadeiras em amostragens desigualmente espaçadas no tempo. Entre os diversos métodos baseados em Tranformadas de Fourier existentes, foram comparados os desempenhos de dois, o CLEAN (Roberts et al., 1987) e o CLEANEST (Foster, 1995), de modo a se optar pelo uso de um deles neste trabalho. É importante destacar que não existe até hoje um método de análise temporal que forneça exatamente as informações desejadas.

A determinação correta de amplitudes, freqüências e fases de sinais periódicos em dados desigualmente espaçados no tempo exige a discriminação e posterior eliminação de picos espúrios que estão presentes no espectro de potência. Tais picos podem ser causados pelo problema da amostragem finita (resolução finita em freqüência) e pelas periodicidades na tomada de dados. Os métodos de análise temporal descritos a seguir baseiam-se em transformadas de Fourier e são utilizadas com freqüência pelo grupo de Sismologia Estelar do IAG-USP (Emilio, 1997). Uma das grandes dificuldades, ainda sem solução, na procura por sinais periódicos ocorre quando duas ou mais freqüências verdadeiras diferem por valores próximos a múltiplos inteiros de $\pm 1 \mathrm{c} / \mathrm{d}$. Nesse caso a 
soma das intensidades de seus aliases pode facilmente tornar-se maior do que as intensidades dos picos centrados nas freqüências verdadeiras.

\subsubsection{O Algoritmo CLEAN}

Esse algoritmo, originalmente usado em sua forma bidimensional para tratamento de imagens (Högbom, 1974), em sua versão unidimensional (Roberts et al. 1987) é uma ferramenta importante na verificação de padrões espúrios associados às freqüências em periodogramas, provocadas por amostras incompletas de dados e por resolução limitada em freqüência devido ao espaçamento finito da série temporal. Assim, o objetivo desse algoritmo é retirar tais padrões espúrios do periodograma de forma iterativa.

Considerando uma série temporal discreta formada por um número finito $\mathrm{N}$ de pontos e representada por uma função $f_{r}$ com $r=1,2,3, \ldots, N$, tal série pode ser entendida como o produto de uma função contínua $\mathrm{f}$ com uma função de amostragem $s(\mathrm{t})$, a qual é a soma ponderada de $\mathrm{N}$ funções Delta de Dirac. Considerando tanto a constante de normalização de $\mathrm{s}(\mathrm{t})$ como o peso para cada ponto como tendo valor unitário (mesmo se não forem unitários, pode-se efetuar a ponderação inicialmente e depois tratar o problema da mesma forma, o que não perde a generalidade), então o sinal amostrado pode ser escrito como:

$$
f_{S}(t)=f(t) s(t)=\left(\frac{1}{N}\right) \sum_{r=1}^{N} f(t) \delta\left(t-t_{r}\right)=\left(\frac{1}{N}\right) \sum_{r=1}^{N} f_{r} \delta\left(t-t_{r}\right)
$$

Pelo Teorema da Convolução de Fourier, a transformada de Fourier do sinal amostrado é a convolução do espectro com a transformada de Fourier da função de amostragem

$$
D(v)=F(v) \otimes W(v)=\int_{-\infty}^{+\infty} F\left(v^{\prime}\right) W\left(v-v^{\prime}\right) d v^{\prime}
$$

onde $\mathrm{D}(v)$ e $\mathrm{W}(v)$ são, respectivamente, o espectro "sujo" (dirty) e a função janela. A idéia do método é extrair a freqüência e seus lobos laterais gradativamente do espectro. Começando com o espectro dirty $\mathrm{D}$, encontramos a amplitude e a freqüência da primeira componente espectral a partir da relação

$$
a \approx \alpha(D, \hat{v})=\frac{D(\hat{v})-D^{*}(\hat{v}) W(2 \hat{v})}{1-|W(2 \hat{v})|^{2}}
$$

Depois, removemos a contribuição dessa componente e seus lobos laterais, subtraindo de $D$ a expressão

$$
D(v)=a W(v-\hat{v})+a^{*} W(v+\hat{v})
$$


com os resultados de (4.14) aplicados a esta. Obtemos então o primeiro resíduo, $\mathrm{R}^{1}$. Essa expressão é multiplicada por um fator ou ganho g, de forma que a subtração se processe aos poucos durante as iterações e sem erros. O procedimento é repetido para os outros resíduos $\mathrm{R}^{2}, \mathrm{R}^{3}, \ldots$ A iteração deve ser encerrada por alguma condição de parada que pode ser: o valor máximo de $\mathrm{R}^{\mathrm{i}}$ é maior que algum ruído pré determinado, ou a soma dos módulos das componentes $C L E A N$ não cresceu de forma significativa. Após determinar todas as componentes espectrais CLEAN, por razões estéticas, elas devem ser convoluídas com uma gaussiana $\mathrm{B}(v)$, para cada componente encontrada que é somada ao último resíduo. Os parâmetros de $\mathrm{B}(v)$ são encontrados através de um ajuste de uma gaussiana no pico central $(v=0)$ da função janela. Descrevendo a seguir, de forma resumida, o algoritmo CLEAN, temos:

1) Cálculo da Transformada de Fourier dos dados;

2) Começando com $\mathrm{i}=1$, determina-se a freqüência $v^{\mathrm{i}}$ da i-ésima componente $C L E A N$ do máximo de $\left|R^{i-1}(v)\right|$; usando ganho $\mathrm{g}$, a amplitude da componente será $c^{i}=g \alpha\left(R^{i-1}, v^{i}\right)$

3) Forma-se o i-ésimo espectro residual $R^{\prime}(v)=R^{i-1}(v)-\left[c^{i} W\left(v-v^{i}\right)+\left(c^{i}\right) * W\left(v+v^{i}\right)\right]$;

4) Examina-se o $\mathrm{R}^{\mathrm{i}}$ e as componentes espectrais $C L E A N$ acumuladas, para verificar se o critério de parada foi atingido. Caso não tenha sido, retorna-se ao segundo passo;

5) Satisfeito o critério de parada, ajusta-se a função feixe $B(v)$ à função janela $\mathrm{W}$;

6) Constrói-se o espectro $C L E A N$ ou periodograma final $S(v)$ convoluindo as $\mathrm{k}$ componentes CLEAN com $B(v)$, e somando-se o espectro residual final $S(v)=\sum_{i=1}^{k}\left[c^{i} B\left(v-v^{i}\right)+\left(c^{i}\right) * B\left(v+v^{i}\right)\right]+R^{k}(v)$

\subsubsection{O Algoritmo CLEANEST}

A idéia central de métodos como o CLEANEST é considerar as séries temporais como vetores que podem ser decompostos através de projeções numa base de um espaço vetorial, formada por outras funções (no caso os vetores são as funções: $|1\rangle,|\sin (\omega t)\rangle$, $|\cos (\omega t)\rangle)$. Além disso esse algoritmo resolve o problema da ortogonalidade entre os vetores da base, fundamental para o cálculo correto de amplitudes e fases (Emílio, 1997). Esse algoritmo é um processo iterativo que vai sequencialmente escolhendo as freqüências mais convenientes para as funções tentativas.

Primeiramente, o algoritmo calcula a TFD dos dados, subtraindo a média dos dados, decompondo assim o vetor resultante numa base ortonormal gerada pelos vetores $|1\rangle,|\sin (\omega t)\rangle,|\cos (\omega t)\rangle$ a partir do método de Gram-Schmidt modificado. Se o maior pico obtido pela transformada for estatisticamente significativo do ponto de vista da Hipótese Nula Básica, isto é, se o valor da variável de Chi-Quadrado $\mathrm{P}_{\mathrm{C}}$ estiver acima do limite inferior de significância, então uma função modelo com a freqüência desse pico será subtraída dos dados (ou resíduo anterior) formando o resíduo $|r\rangle$. Então o espectro 
de potência é definido como a soma dos quadrados dos coeficientes do vetor modelo escrito nessa base ortonormal.

Em seguida, o algoritmo calcula a TFD do resíduo e determina o próximo pico estatisticamente significativo. Depois disso ele volta aos dados originais para projetá-los agora numa base que utiliza como valores iniciais as freqüências obtidas anteriormente e mais a última freqüência determinada. $\mathrm{O}$ algoritmo procura então pelo valor máximo de $\mathrm{P}_{\mathrm{C}}$ (que é uma variável de Chi-Quadrado com $3 \mathrm{n}$ graus de liberdade e valor esperado 1) para diversos conjuntos de freqüências com valores próximos aos encontrados anteriormente, sendo esse máximo um máximo local, uma vez que procuramos por freqüências dentro de um domínio limitado. Depois de encontrar o conjunto otimizado de freqüências, forma-se outra vez uma nova função modelo e depois o algoritmo subtrai essa nova função modelo dos dados. Esse processo iterativo continua até que não existam mais picos estatisticamente significativos ou então até que se tenha atingido um número máximo de freqüências utilizado como condição de parada. Ao final do processo teremos uma função modelo constituindo uma base formada por $2 n+1$ funções tentativa, permitindo também que as $\mathrm{n}$ freqüências sejam levemente ajustadas a cada iteração. A Hipótese Nula Básica sobre a qual se apoia o critério de escolha de freqüências é o seguinte:

a) Os dados são compostos apenas por um sinal constante mais erros;

b) Cada um dos erros é uma variável aleatória normal, resultado de um processo estocástico;

c) Se os dados tiverem variâncias $\sigma_{\mathrm{r}}^{2}$ diferentes, os pesos serão dados por $\mathrm{p}_{\mathrm{r}}=\sigma_{\mathrm{r}}^{-2}$;

d) Os erros não estão correlacionados (são independentes).

\subsection{3. Álgebra do Algoritmo CLEANEST}

Uma série temporal de $\mathrm{N}$ dados igualmente espaçados pode ser representada como um vetor de dados com a forma $|x\rangle=\left[x\left(t_{1}\right), x\left(t_{2}\right), \ldots, x\left(t_{N}\right)\right]$. Uma função qualquer no tempo $f(\mathrm{t})$ é definida por um vetor $|f\rangle=\left[f\left(t_{1}\right), f\left(t_{2}\right), \ldots, f\left(t_{N}\right)\right]$. As funções tentativa dependem do tempo e são (Foster, 1995):

$$
\begin{aligned}
& \phi_{1}=1(t) \\
& \phi_{2}=\sqrt{2} \sin \left(\omega_{k} t\right) \\
& \phi_{3}=\sqrt{2} \cos \left(\omega_{k} t\right)
\end{aligned}
$$

onde:
a) $\omega_{k}=\frac{2 \pi}{T} k$
b) $k$ é um inteiro $1 \leq k \leq \frac{1}{2} N$
c) $T$ é o comprimento total dos dados em tempo: $T=N \Delta$ 
A função $\phi_{1}=1(t)$ é a função unitária $|1\rangle=[1,1, \ldots, 1]$. O tempo das observações é colocado de maneira indexada: $t_{\alpha}=\alpha \Delta$. Os vetores tentativa serão da forma:

$\left|\phi_{1}\right\rangle=\left[1\left(t_{1}\right), 1\left(t_{2}\right), \ldots, 1\left(t_{N}\right)\right]$

$\left|\phi_{2}\right\rangle=\left\lfloor\sqrt{2} \sin \left(\omega_{k} t_{1}\right), \sqrt{2} \sin \left(\omega_{k} t_{2}\right), \ldots, \sqrt{2} \sin \left(\omega_{k} t_{N}\right)\right\rfloor$

$\left|\phi_{3}\right\rangle=\left\lfloor\sqrt{2} \cos \left(\omega_{k} t_{1}\right), \sqrt{2} \cos \left(\omega_{k} t_{2}\right), \ldots, \sqrt{2} \cos \left(\omega_{k} t_{N}\right)\right\rfloor$

Os produtos internos entre esses vetores obedecem os critérios de ortogonalidade:

$$
\begin{aligned}
& \langle 1 \mid 1\rangle=1 \\
& \left\langle 1 \mid \sqrt{2} \sin \left(\omega_{k} t\right)\right\rangle=0 \\
& \left\langle 1 \mid \sqrt{2} \cos \left(\omega_{k} t\right)\right\rangle=0 \\
& \left\langle\sqrt{2} \sin \left(\omega_{i} t\right) \mid \sqrt{2} \sin \left(\omega_{j} t\right)\right\rangle=\delta_{i j} \\
& \left\langle\sqrt{2} \cos \left(\omega_{i} t\right) \mid \sqrt{2} \cos \left(\omega_{j} t\right)\right\rangle=\delta_{i j} \\
& \left\langle\sqrt{2} \sin \left(\omega_{i} t\right) \mid \sqrt{2} \cos \left(\omega_{j} t\right)\right\rangle=1
\end{aligned}
$$

onde $\delta_{i j}=0$ se $\mathrm{i}=\mathrm{j}$ e $\delta_{i j}=1$ se $\mathrm{i} \neq \mathrm{j}$. Os vetores tentativa formam uma base ortonormal completa. A matriz $S$ é o tensor métrico para o espaço modelo quando usamos as funções tentativa como base: $S_{a b}=\left\langle\phi_{a} \mid \phi_{b}\right\rangle$. Ela é uma matriz identidade, bem como sua inversa, e então:

$$
S_{a b}=\left[\begin{array}{lll}
\left\langle\phi_{1} \mid \phi_{1}\right\rangle & \left\langle\phi_{1} \mid \phi_{2}\right\rangle & \left\langle\phi_{1} \mid \phi_{3}\right\rangle \\
\left\langle\phi_{2} \mid \phi_{1}\right\rangle & \left\langle\phi_{2} \mid \phi_{2}\right\rangle & \left\langle\phi_{2} \mid \phi_{3}\right\rangle \\
\left\langle\phi_{3} \mid \phi_{1}\right\rangle & \left\langle\phi_{3} \mid \phi_{2}\right\rangle & \left\langle\phi_{3} \mid \phi_{3}\right\rangle
\end{array}\right]=\left[\begin{array}{ccc}
1 & 0 & 0 \\
0 & 1 & 0 \\
0 & 0 & 1
\end{array}\right]
$$

O objetivo aqui é encontrar os coeficientes do vetor modelo. Isso é feito invertendo-se a matriz dos coeficientes $S_{a b}$, e o vetor modelo será:

$$
y_{a}=\sum_{b} S_{a b}^{\prime-1}\left\langle\phi_{b} \mid x\right\rangle=\frac{\sum_{b a} S_{a b}^{\prime-1} \widetilde{\omega}_{a} \phi_{b}\left(t_{a}\right) x\left(t_{a}\right)}{\sum_{\lambda} \widetilde{\omega}_{\lambda}}
$$


onde $\widetilde{\omega}_{a}$ é um peso estatístico do dado em cada ponto $a$. Para uma determinada freqüência, nosso vetor modelo ficará com o formato:

$|y\rangle=\sum_{a} y_{a}\left|\phi_{a}\right\rangle=y_{1}\left|\phi_{1}\right\rangle+y_{2}\left|\phi_{2}\right\rangle+y_{3}\left|\phi_{3}\right\rangle$

A norma quadrática será dada por:

$$
Q=\langle y \mid y\rangle=2\left(\left\langle\cos \left(\omega_{k} t\right) \mid x\right\rangle^{2}+\left\langle\sin \left(\omega_{k} t\right) \mid x\right\rangle^{2}\right)
$$

A potência da projeção desse vetor será então (Foster, 1995):

$$
P=\frac{N V_{y}}{2 f \cdot s^{2}}
$$

$\mathrm{O}$ fator $2 f$ representa o número de funções tentativa a menos da função constante, sendo uma distribuição de $\chi^{2} \operatorname{com} 2 f$ graus de liberdade e valor esperado 1. A variância estimada $s^{2}$ é calculada por $s^{2}=\frac{N V_{x}}{(N-1)}$, onde $V_{x}=\langle x \mid x\rangle-\langle 1 \mid x\rangle^{2}$ é a variação dos dados e $\bar{x}=\langle 1 \mid x\rangle$ é a média dos dados. 


\subsubsection{Comparação do Desempenho dos Algoritmos}

Para realizar uma comparação entre os métodos de análise temporal, optei por testar os algoritmos com sinais artificiais. A análise com sinais artificiais é interessante pois pode-se visualizar melhor a precisão com que os algoritmos determinam os períodos, uma vez que eles já são conhecidos.

Inicialmente, foram confeccionados dados com sinais senoidais amostrados igualmente no tempo. As freqüências que escolhi para esses sinais de entrada foram as de $7.2 \mathrm{c} / \mathrm{d}, 9.9 \mathrm{c} / \mathrm{d}$ e $11.9 \mathrm{c} / \mathrm{d}$, e suas combinações: $7.2 \mathrm{c} / \mathrm{d}, 11.9 \mathrm{c} / \mathrm{d}, 7.2+11.9 \mathrm{c} / \mathrm{d}$ e $7.2+$ $9.9+11.9 \mathrm{c} / \mathrm{d}$. Cada uma das freqüências foi injetada com amplitude $\mathrm{A}=50$. No caso de sinais desigualmente espaçados no tempo, optei por utilizar uma janela de observação real (observação de $\eta$ Centauri no LNA nos dias 30/05, 31/05, 01/06 e 02/06/1996). As simulações denominadas Janela Menor são constituídas de 135 pontos igualmente espaçados, as denominadas Janela Maior são constituídas de 405 pontos igualmente espaçados (a Janela Maior é 3 vezes mais extensa que a Janela Menor). As simulações feitas com janela de observação real são constituídas de 135 pontos.

Quanto aos ruídos introduzidos nos dados, estes são basicamente números aleatórios gerados com o pacote IDL. As seqüências de números aleatórios foram criadas de forma a possuírem uma distribuição gaussiana de probabilidades com média nula e desvio padrão $\sigma$. Assim, aumentando a variância da distribuição dos pontos aleatórios, aumentamos o nível de ruído nos dados. A escolha de distintos níveis de ruído foi feita visando mostrar a variação da performance dos algoritmos à medida em que o sinal se torna mais fraco (isto é, o ruído aumenta progressivamente). Observacionalmente, isto pode significar uma noite em que todos os espectros foram tomados com alta relação sinal/ruído, depois outra noite onde o sinal se torna mais fraco devido à presença de nuvens, etc. No caso em que o desvio padrão do ruído $\sigma=87.2$, temos uma série de números aleatórios compreendidos num intervalo de $\sim-240 \leq \mathrm{x} \leq 240$. Assim, se um desses números aleatórios valer $\sim 240$, então ele será cerca de 5 vezes maior do que o sinal de cada freqüência injetada. As unidades utilizadas para a variável dependente nas simulações são as unidades usuais empregadas para espectros normalizados, no caso a intensidade relativa à intensidade do contínuo $\left(\mathrm{I} / \mathrm{I}_{0}\right)$, que é uma grandeza adimensional. No caso do estudo de Sismologia Estelar, a medida mais importante em um espectro é a posição dos bumps em velocidade ao longo do perfil de linha, e não em fluxo, daí a escolha da unidade como intensidade relativa e não fluxo relativo.

Além dos casos mencionados, foi analisado também o caso em que a série temporal apresentou ruído caracterizado por variância distinta a cada medida realizada. A justificativa para isso é que cada espectro é medido em instantes de tempo sucessivos, na presença de condições climáticas distintas, o que pode fazer com que a intensidade da luz proveniente da estrela varie com o tempo. 


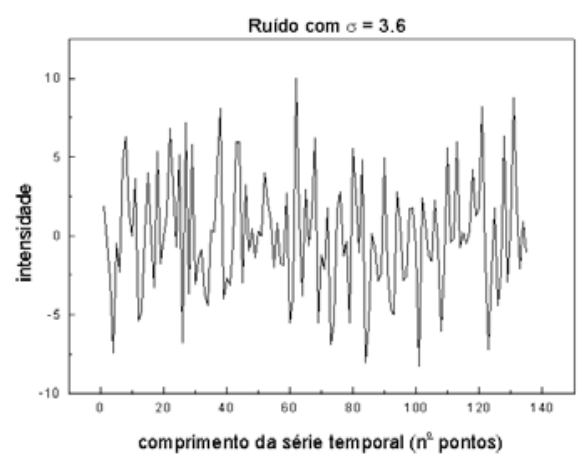

a) Distribuição com $\sigma=3.6$

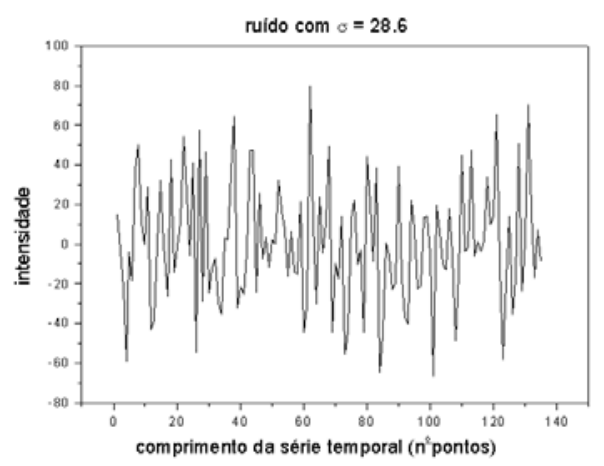

c) Distribuição com $\sigma=28.6$

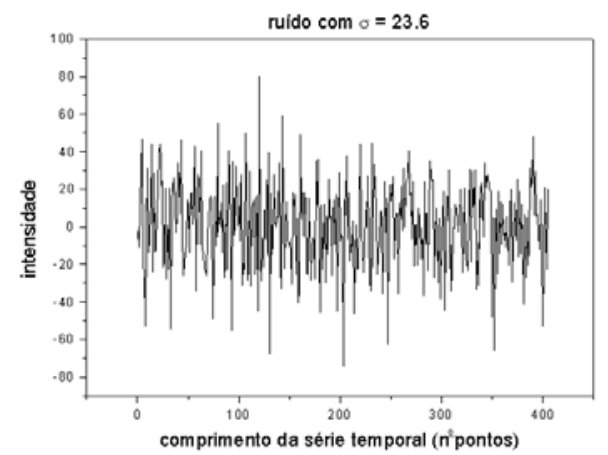

b) Distribuição com $\sigma=23.6$

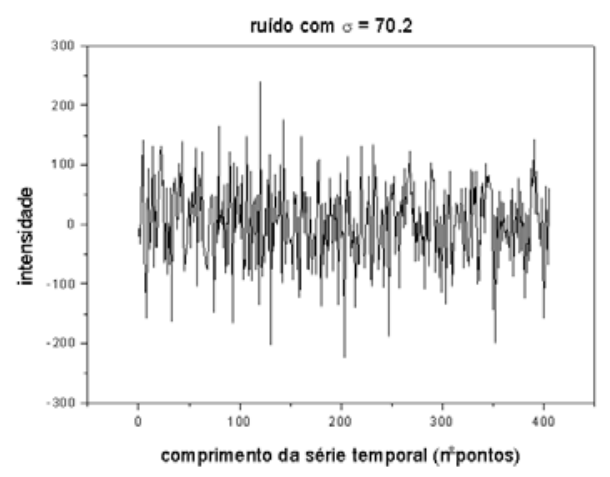

d) Distribuição com $\sigma=70.2$

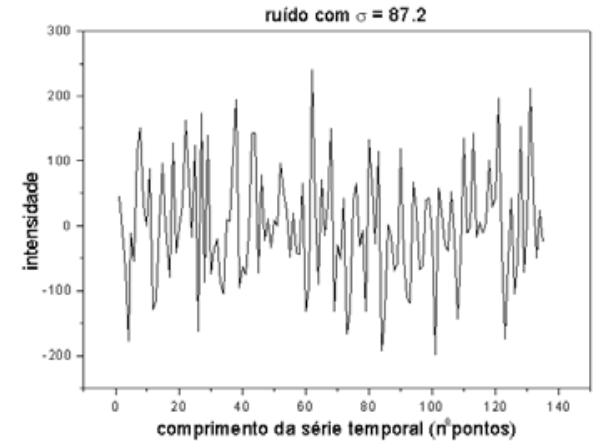

e) Distribuição com $\sigma=87.2$

Fig.12. Gráficos dos pontos aleatórios introduzidos nos dados sintéticos.

A seguir, de forma a dificultar mais a identificação das freqüências, é construída uma nova série de números aleatórios, onde cada ponto é gerado segundo uma distribuição normal diferente. Esse novo ruído será utilizado mais adiante, nas séries temporais com sinais compostos de senóides com amplitudes diferentes. 


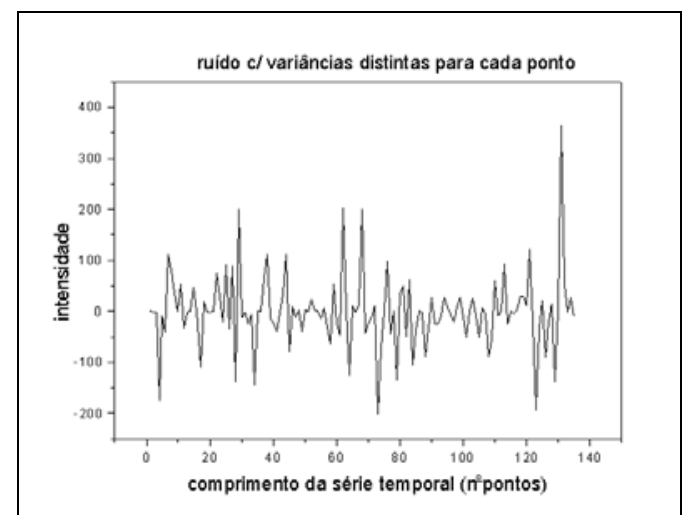

Fig.13. Pontos gerados segundo distribuições de probabilidades distintas (uma para cada ponto).

A seguir são exibidos os dados artificiais gerados e sua análise, utilizando-se os algoritmos CLEAN e CLEANEST. A comparação será feita em tabelas apresentadas na seqüência. No caso de dados igualmente espaçados no tempo, serão usados dois tipos de janelas de observação, onde uma é três vezes maior que a outra (janela menor e janela maior). Com isso, pode-se ilustrar o desempenho do CLEAN e do CLEANEST em determinar as freqüências a partir de dados com um número de ondas amostradas maior ou menor. No caso de dados desigualmente espaçados no tempo, a janela de observação corresponde à janela dos dados reais de espectroscopia constantes neste trabalho e referentes às noites de 30,31/05 e 01,02/06/1996. Com relação às amplitudes dos sinais de entrada, temos dois casos distintos, o caso 1, onde as amplitudes dos sinais de entrada são todas iguais a 50, e o caso 2 onde as amplitudes para cada freqüência variam.

O caso em que as amplitudes dos sinais de entrada são iguais é o caso mais simples a ser estudado. A intensidade do ruído introduzido será medida a partir do desvio padrão $\sigma$ da distribuição de probabilidades que rege os pontos aleatórios gerados, uma vez que, como as séries temporais são sintéticas, torna-se complicado determinar a relação sinal/ruído, pois $\mathrm{S} / \mathrm{R}$ é medida para cada espectro em relação ao seu contínuo (nós só temos um ponto por "espectro" nos dados sintéticos). Para dar uma idéia, no caso em que $\sigma=87$, a valor de um número aleatório que constitui o ruído pode chegar a ser até quase 5 vezes maior do que a amplitude do sinal introduzido (para $\mathrm{A}=50$ ). Em todos os casos os sinais de entrada são sinais senoidais com a forma:

$$
f(t)=\sum_{i=1}^{3} A_{i} \sin \left(2 \pi v_{i} \mathrm{t}\right)
$$

onde a fase injetada em todos os casos foi zero.

Nos testes a seguir, a freqüência mínima utilizada nas pesquisas com o CLEAN e o CLEANEST foi, em todos os casos, $2.5 \mathrm{c} / \mathrm{d}$, a freqüência máxima foi $15 \mathrm{c} / \mathrm{d}$ e o passo $1 / 1000$ da freqüência mínima $(0.0025 \mathrm{c} / \mathrm{d})$. Tais condições iniciais são importantes pois os resultados obtidos com a utilização dos algoritmos variam com a freqüência mínima escolhida e com o passo utilizado. 
Caso 1 - Senóides com Amplitudes Iguais

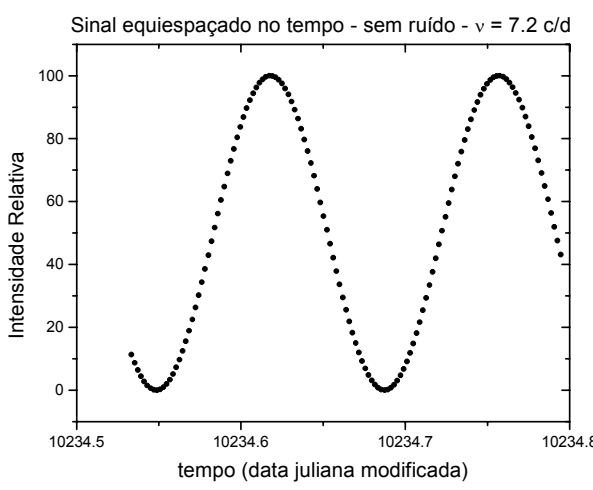

a) sinal $\mathrm{c} / v_{\text {entrada }}=7.2 \mathrm{c} / \mathrm{d}$

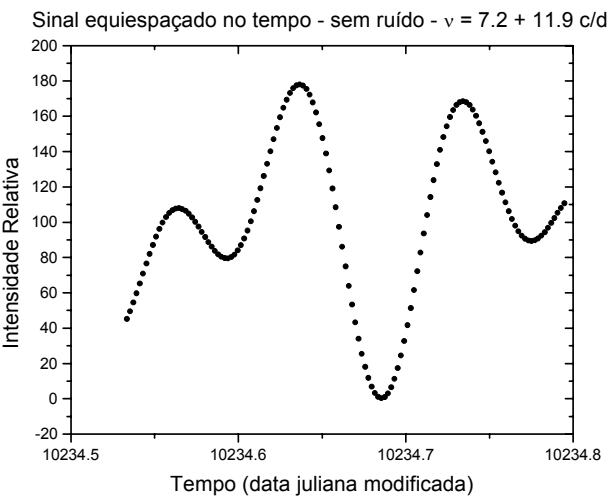

c) sinal c/ $v_{\text {entrada }}=7.2+11.9 \mathrm{c} / \mathrm{d}$

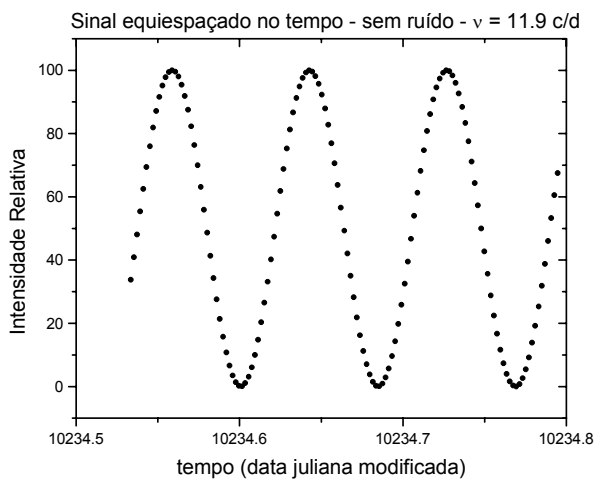

b) sinal c/ $v_{\text {entrada }}=11.9 \mathrm{c} / \mathrm{d}$

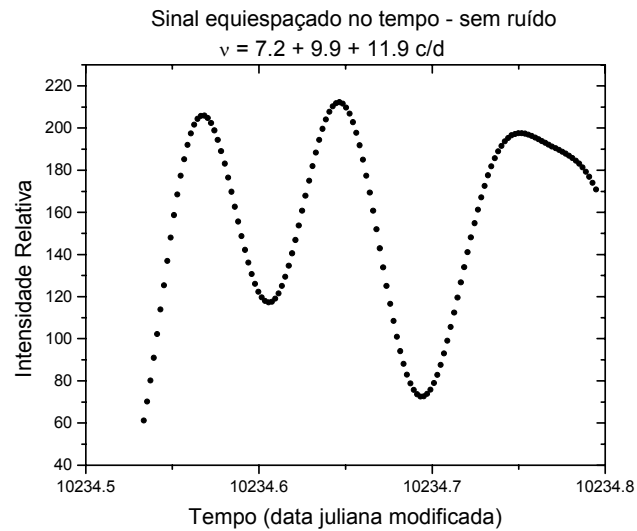

d) sinal c/ $v_{\text {entrada }}=7.2+9.9+11.9 \mathrm{c} / \mathrm{d}$

Fig.14. Sinais igualmente espaçados no tempo e sem ruído (Janela menor).

Os resultados das análises com os algoritmos CLEAN e CLEANEST dos sinais de entrada da Figura 14 podem ser vistos na Tabela 5.

\begin{tabular}{|c|c|c|}
\hline $\begin{array}{c}\text { Freqüências de } \\
\text { Entrada (ciclos/d) } \\
\text { Freq./(amplitude) }\end{array}$ & $\begin{array}{c}\text { Janela menor } \\
\text { CLEAN } \\
\text { Freq. / (amplitude) }\end{array}$ & $\begin{array}{c}\text { Janela menor } \\
\text { CLEANEST } \\
\text { Freq. / (amplitude) }\end{array}$ \\
\hline $7.2 /(50)$ & $7.1000 /(52)$ & $7.2000 /(50)$ \\
\hline $11.9 /(50)$ & $11.7675 /(52)$ & $11.9000 /(50)$ \\
\hline $7.2+11.9$ & $8.5505 /(52)$ & $7.2000 /(50)$ \\
$(50+50)$ & $12.8725 /(52)$ & $11.9000 /(50)$ \\
\hline $7.2+9.9+11.9$ & $11.1050 /(43)$ & $7.1750 /(45)$ \\
$(50+50+50)$ & $6.2300 /(46)$ & $10.9320 /(44)$ \\
\hline
\end{tabular}

Tab. 5. Testes sem ruído com janela de observação menor. 


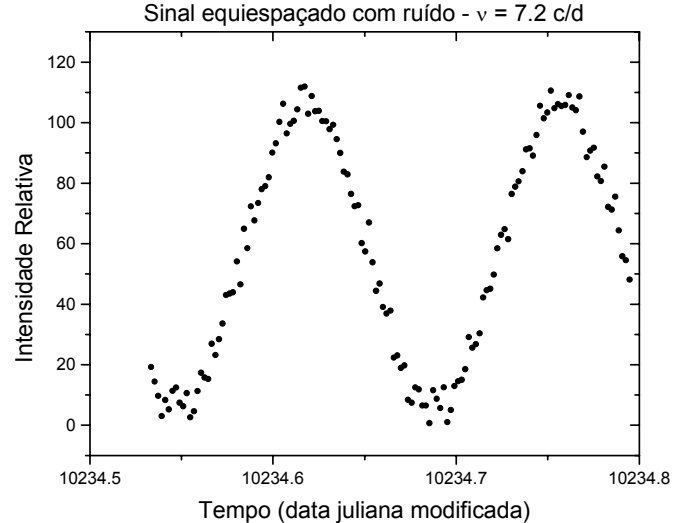

a) sinal $\mathrm{c} / \mathrm{v}_{\text {entrada }}=7.2 \mathrm{c} / \mathrm{d}$

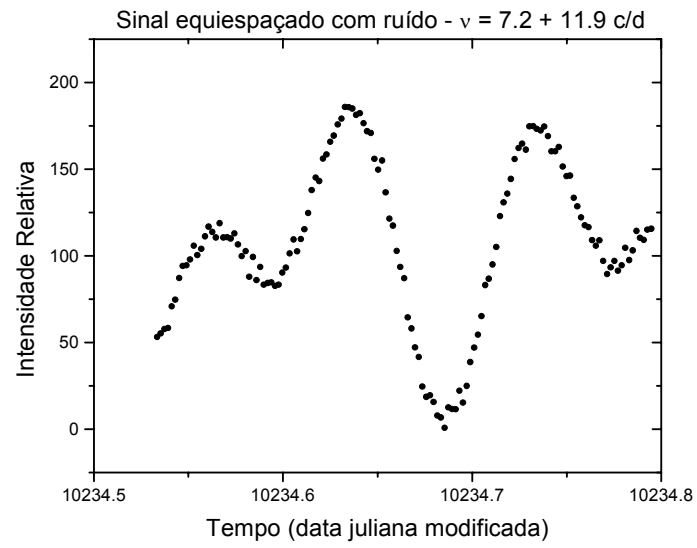

c) sinal c/ $v_{\text {entrada }}=7.2+11.9 \mathrm{c} / \mathrm{d}$

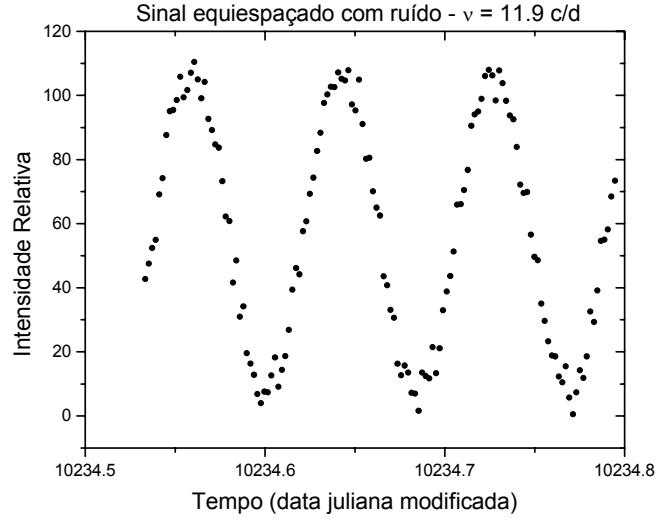

b) sinal c/ $v_{\text {entrada }}=11.9 \mathrm{c} / \mathrm{d}$

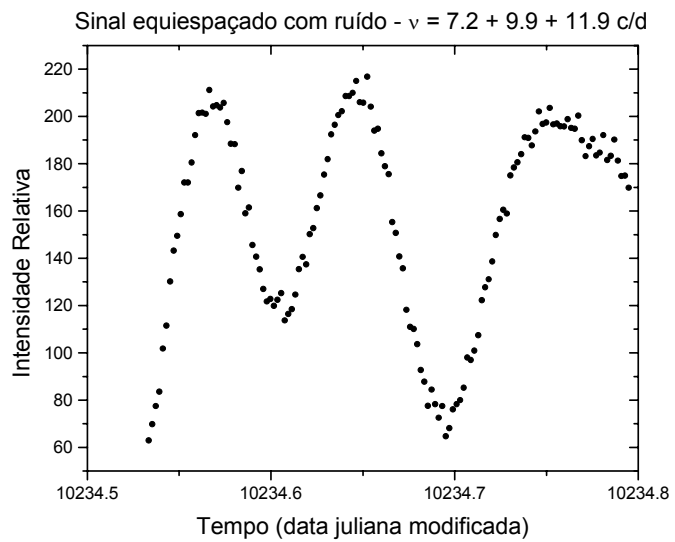

d) sinal $\mathrm{c} / \mathrm{v}_{\text {entrada }}=7.2+9.9+11.9 \mathrm{c} / \mathrm{d}$

Fig.15. Sinais igualmente espaçados no tempo e com ruído $\sigma=3.6$

\begin{tabular}{|c|c|c|}
\hline $\begin{array}{c}\text { Freqüências de } \\
\text { Entrada (ciclos/d) } \\
\text { Freq./(amplitude) }\end{array}$ & $\begin{array}{c}\text { Janela menor } \\
\text { CLEAN } \\
\text { Freq. / (amplitude) }\end{array}$ & $\begin{array}{c}\text { Janela menor } \\
\text { CLEANEST } \\
\text { Freq. / (amplitude) }\end{array}$ \\
\hline $7.2 /(50)$ & $7.1000 /(54)$ & $7.2000 /(50)$ \\
\hline $11.9 /(50)$ & $11.8000 /(52)$ & $11.9000 /(50)$ \\
\hline $\begin{array}{l}7.2+11.9 \\
(50+50)\end{array}$ & $\begin{array}{c}8.5000 /(52) \\
12.8000 /(55) \\
\end{array}$ & $\begin{array}{c}8.5500 /(48) \\
12.8000 /(47) \\
\end{array}$ \\
\hline $\begin{array}{c}7.2+9.9+11.9 \\
(50+50+50)\end{array}$ & $\begin{array}{c}6.2000 /(47) \\
11.1000 /(44)\end{array}$ & $\begin{array}{c}6.2000 /(47) \\
11.0000 /(45)\end{array}$ \\
\hline
\end{tabular}

Tab. 6. Testes com ruído $\sigma=3.6$ e janela de observação menor. 

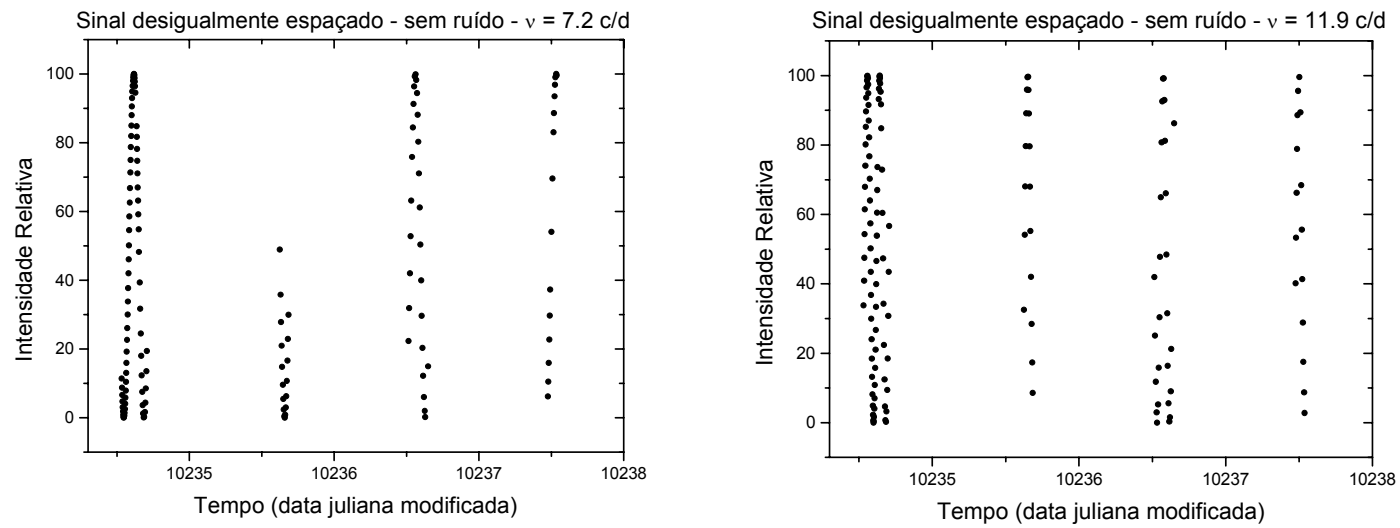

a) sinal $\mathrm{c} / v_{\text {entrada }}=7.2 \mathrm{c} / \mathrm{d}$

b) sinal $\mathrm{c} / \mathrm{v}_{\text {entrada }}=11.9 \mathrm{c} / \mathrm{d}$
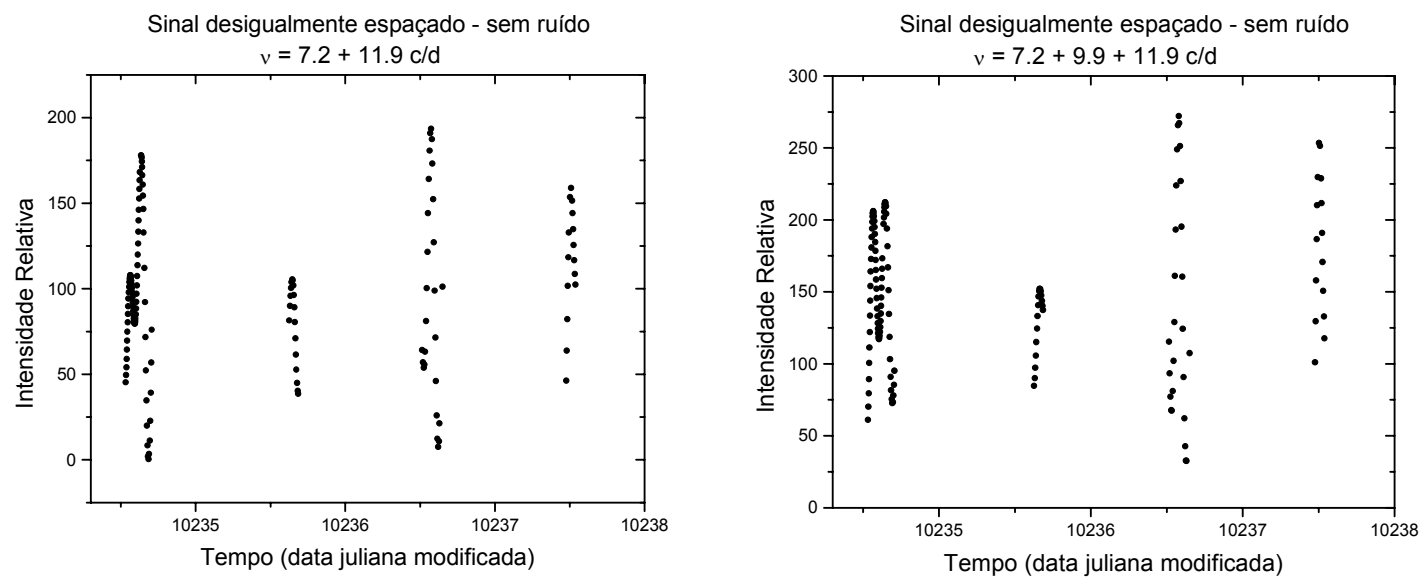

c) sinal $\mathrm{c} / \mathrm{v}_{\text {entrada }}=7.2+11.9 \mathrm{c} / \mathrm{d}$

d) sinal c/ $v_{\text {entrada }}=7.2+9.9+11.9 \mathrm{c} / \mathrm{d}$

Fig.16. Sinais desigualmente espaçados no tempo e sem ruído

\begin{tabular}{|c|c|c|}
\hline $\begin{array}{c}\text { Freqüências de } \\
\text { Entrada (ciclos/d) } \\
\text { Freq./(amplitude) }\end{array}$ & $\begin{array}{c}\text { CLEAN } \\
\text { Freq. / (amplitude) }\end{array}$ & $\begin{array}{c}\text { CLEANEST } \\
\text { Freq. / (amplitude) }\end{array}$ \\
\hline $7.2 /(50)$ & $7.2000 /(54)$ & $7.2000 /(50)$ \\
\hline $11.9 /(50)$ & $11.9000 /(52)$ & $11.9000 /(50)$ \\
\hline $7.2+11.9$ & $7.2000 /(48)$ & $7.2000 /(50)$ \\
$(50+50)$ & $11.9100 /(48)$ & $11.9000 /(52)$ \\
\hline $7.2+9.9+11.9$ & $11.9249 /(64)$ & $11.9176 /(62)$ \\
$(50+50+50)$ & $7.2046 /(56)$ & $7.1920 /(50)$ \\
& $9.8782 /(48)$ & $9.9038 /(60)$ \\
\hline
\end{tabular}

Tab. 7. Testes sem ruído em dados desigualmente espaçados no tempo, com a janela de observação correspondente às observações de $\eta$ Cen em 30, 31/05 e 01, 02/06/1996. 

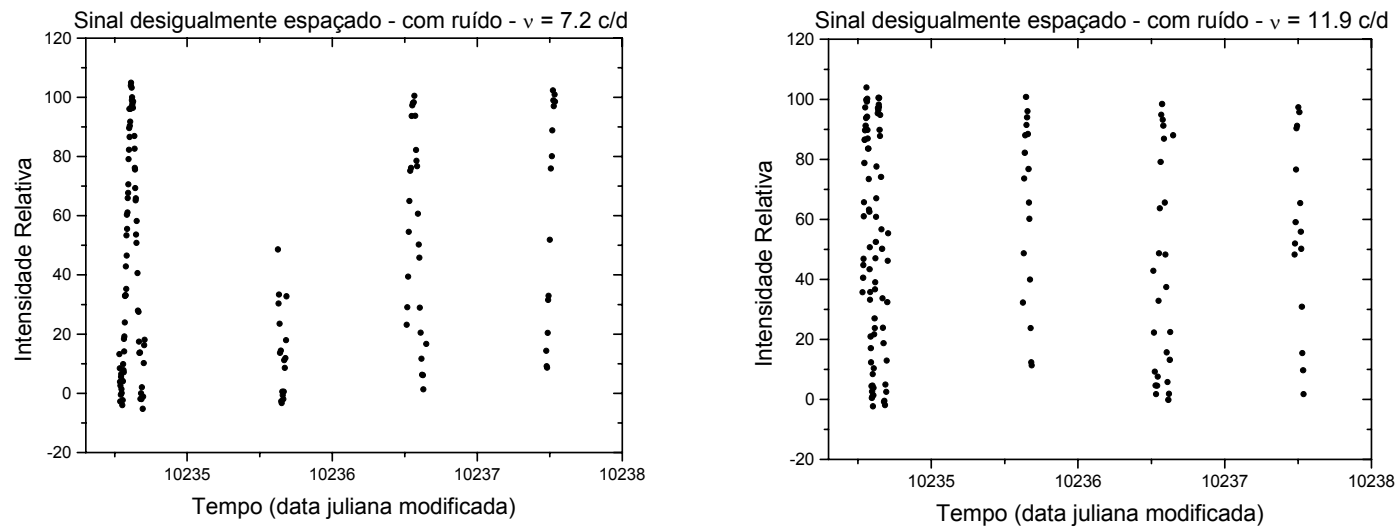

a) sinal $\mathrm{c} / v_{\text {entrada }}=7.2 \mathrm{c} / \mathrm{d}$

b) sinal $\mathrm{c} / \mathrm{v}_{\text {entrada }}=11.9 \mathrm{c} / \mathrm{d}$
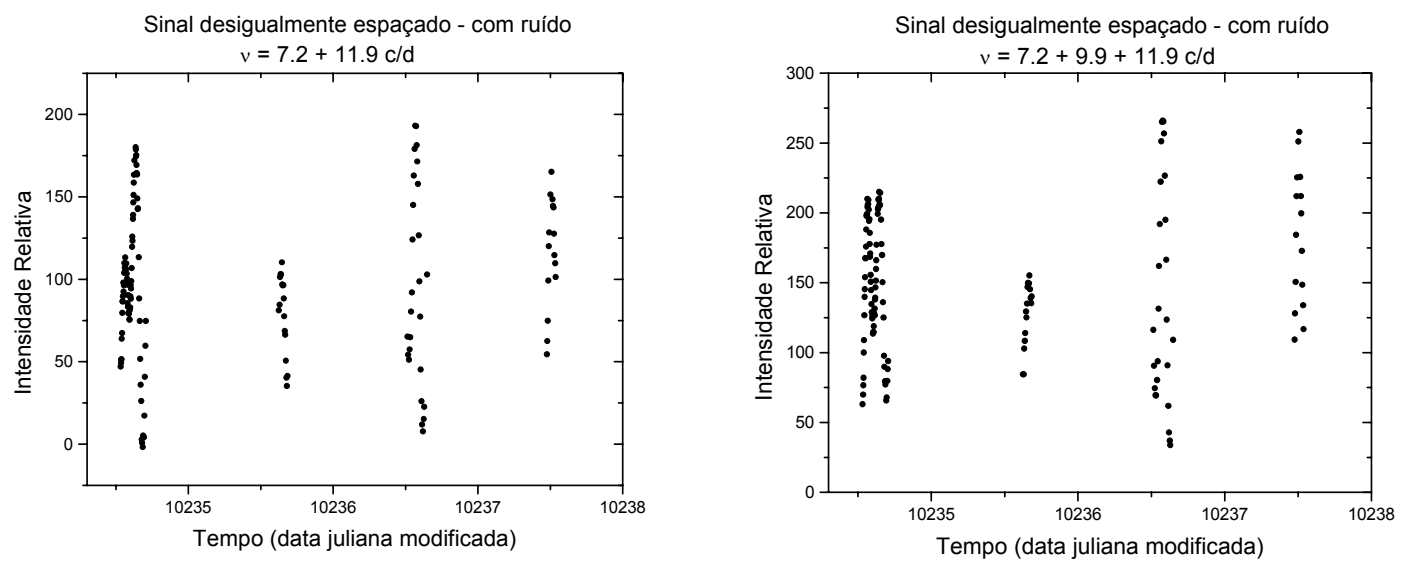

c) sinal $\mathrm{c} / v_{\text {entrada }}=7.2+11.9 \mathrm{c} / \mathrm{d}$

d) sinal $\mathrm{c} / v_{\text {entrada }}=7.2+9.9+11.9 \mathrm{c} / \mathrm{d}$

Fig.17. Sinais desigualmente espaçados no tempo e com ruído $\sigma=3.6$

\begin{tabular}{|c|c|c|}
\hline $\begin{array}{c}\text { Freqüências de } \\
\text { Entrada (ciclos/d) } \\
\text { Freq./(amplitude) }\end{array}$ & $\begin{array}{c}\text { CLEAN } \\
\text { Freq. / (amplitude) }\end{array}$ & $\begin{array}{c}\text { CLEANEST } \\
\text { Freq. / (amplitude) }\end{array}$ \\
\hline $7.2 /(50)$ & $7.2000 /(54)$ & $7.2000 /(50)$ \\
\hline $11.9 /(50)$ & $11.9100 /(52)$ & $11.9000 /(50)$ \\
\hline $7.2+11.9$ & $7.2300 /(48)$ & $7.2000 /(50)$ \\
$(50+50)$ & $11.9100 /(41)$ & $12.9000 /(43)$ \\
\hline $7.2+9.9+11.9$ & $11.9200 /(64)$ & $11.9000 /(55)$ \\
$(50+50+50)$ & $7.2000 /(34)$ & $7.2000 /(50)$ \\
& $9.8700 /(26)$ & $10.9000 /(61)$ \\
\hline
\end{tabular}

Tab. 8. Testes com ruído $\sigma=3.6$ e dados desigualmente espaçados no tempo, com a janela de observação correspondente às observações de ๆCen em 30, 31/05 e 01, 02/06/1996. 


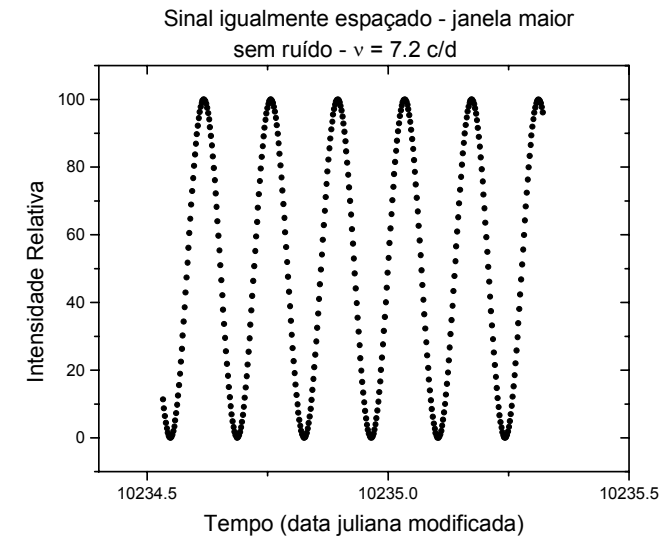

a) sinal de entrada com $7.2 \mathrm{c} / \mathrm{d}$

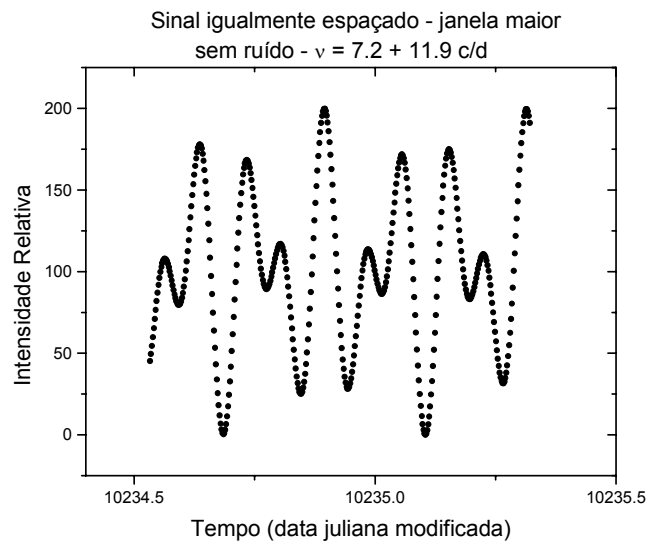

c) sinal de entrada com $7.2+11.9 \mathrm{c} / \mathrm{d}$

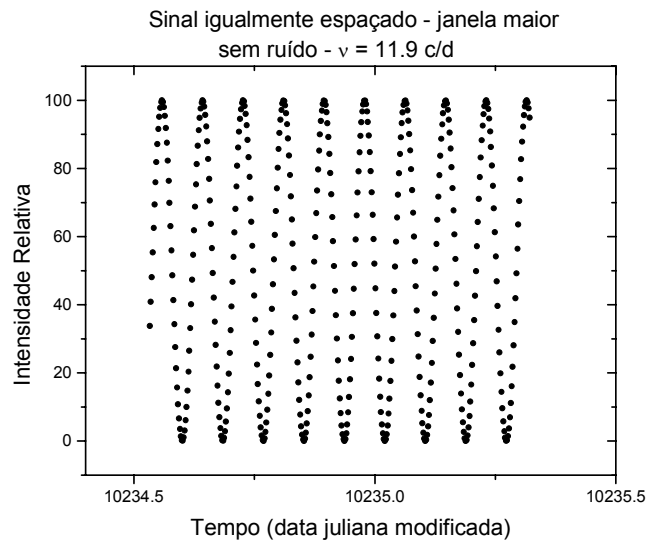

b) sinal de entrada com $11.9 \mathrm{c} / \mathrm{d}$

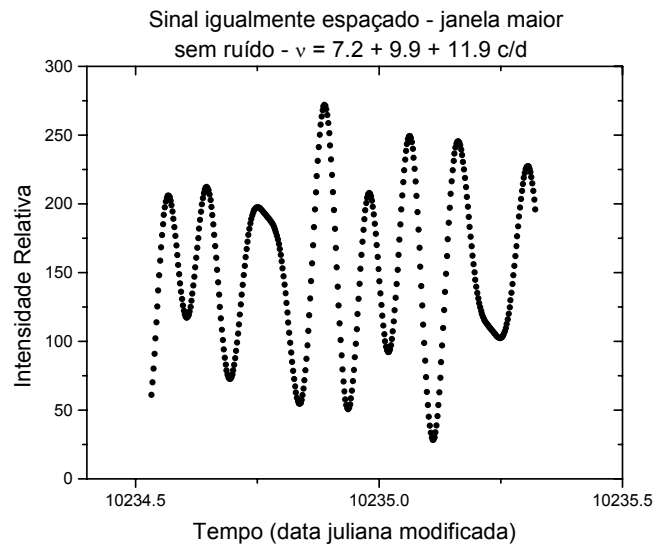

d) sinal de entrada com $7.2+9.9+11.9 \mathrm{c} / \mathrm{d}$

Fig.18. Sinais igualmente espaçados no tempo, janela maior, sem ruído

\begin{tabular}{|c|c|c|}
\hline $\begin{array}{c}\text { Freqüências de } \\
\text { Entrada (ciclos/d) } \\
\text { Freq./(amplitude) }\end{array}$ & $\begin{array}{c}\text { Janela maior } \\
\text { CLEAN } \\
\text { Freq. / (amplitude) }\end{array}$ & $\begin{array}{c}\text { Janela maior } \\
\text { CLEANEST } \\
\text { Freq. / (amplitude) }\end{array}$ \\
\hline $7.2 /(50)$ & $7.2110 /(50)$ & $7.2000 /(50)$ \\
\hline $11.9 /(50)$ & $11.8975 /(50)$ & $11.8950 /(50)$ \\
\hline $7.2+11.9$ & $7.1955 /(52)$ & $7.1850 /(50)$ \\
$(50+50)$ & $11.9120 /(52)$ & $11.8875 /(50)$ \\
\hline $7.2+9.9+11.9$ & $7.2595 /(51)$ & $7.2000 /(50)$ \\
$(50+50+50)$ & $9.8825 /(53)$ & $9.9000 /(50)$ \\
& $11.9870 /(50)$ & $11.9000 /(50)$ \\
\hline
\end{tabular}

Tab. 9. Testes sem ruído em dados igualmente espaçados no tempo, com janela maior. Neste caso, as freqüências são melhor determinadas do que no caso de janela simples. 


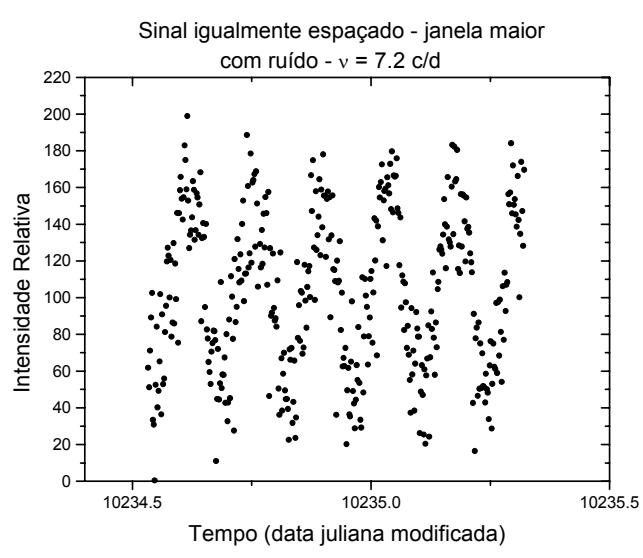

a) sinal de entrada com $7.2 \mathrm{c} / \mathrm{d}$

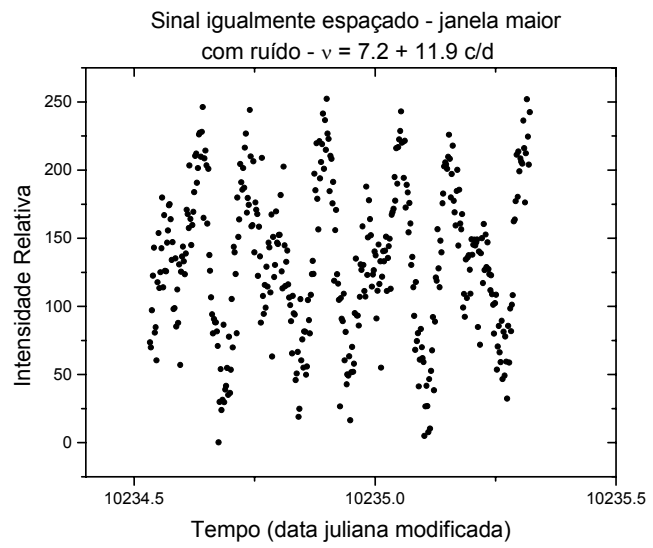

c) sinal de entrada com $7.2+11.9 \mathrm{c} / \mathrm{d}$

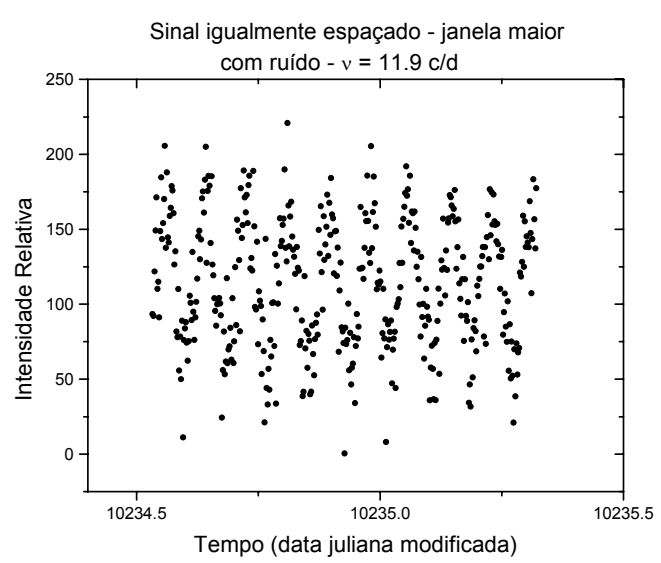

b) sinal de entrada com $11.9 \mathrm{c} / \mathrm{d}$

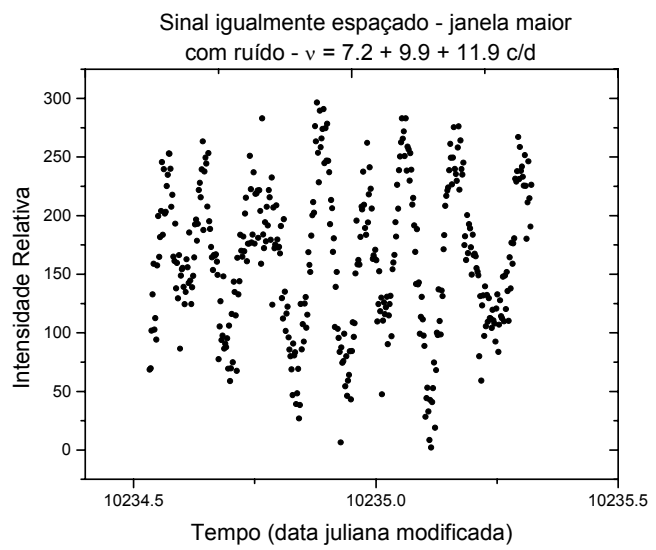

d) sinal de entrada com $7.2+9.9+11.9 \mathrm{c} / \mathrm{d}$

Fig.19. Sinais igualmente espaçados no tempo, janela maior, com ruído $\sigma=23$

\begin{tabular}{|c|c|c|}
\hline $\begin{array}{c}\text { Freqüências de } \\
\text { Entrada (ciclos/d) } \\
\text { Freq./(amplitude) }\end{array}$ & $\begin{array}{c}\text { Janela maior } \\
\text { CLEAN } \\
\text { Freq. / (amplitude) }\end{array}$ & $\begin{array}{c}\text { Janela maior } \\
\text { CLEANEST } \\
\text { Freq. / (amplitude) }\end{array}$ \\
\hline $7.2 /(50)$ & $7.1900 /(53)$ & $7.1800 /(52)$ \\
\hline $11.9 /(50)$ & $11.9000 /(54)$ & $11.9000 /(50)$ \\
\hline $7.2+11.9$ & $7.1700 /(52)$ & $7.1800 /(51)$ \\
$(50+50)$ & $11.9000 /(53)$ & $11.9000 /(50)$ \\
\hline $7.2+9.9+11.9$ & $7.2000 /(53)$ & $7.2000 /(51)$ \\
$(50+50+50)$ & $9.9000 /(55)$ & $9.9000 /(50)$ \\
& $12.0000 /(54)$ & $11.9300 /(51)$ \\
\hline
\end{tabular}

Tab. 10. Testes com ruído $\sigma=23$ em dados igualmente espaçados no tempo, com janela maior. 


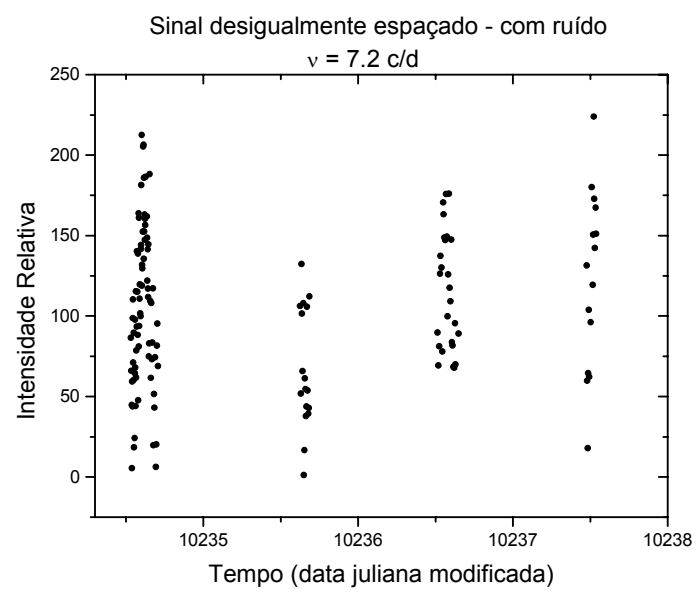

a) sinal de entrada com $7.2 \mathrm{c} / \mathrm{d}$

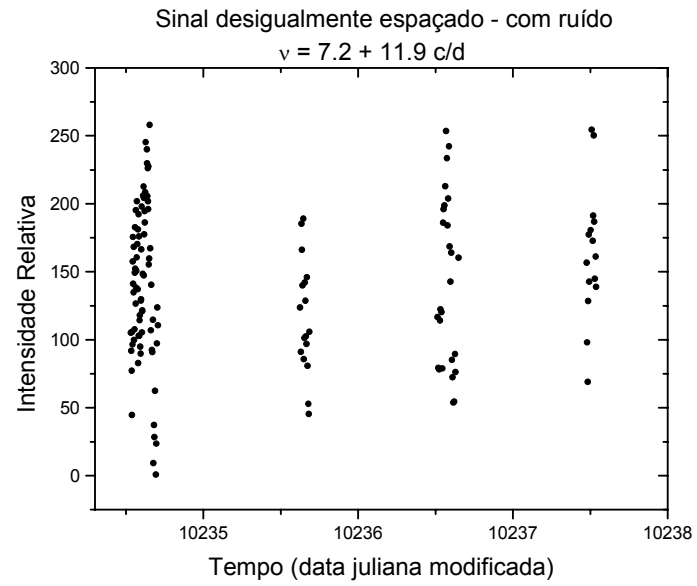

c) sinal de entrada com $7.2+11.9 \mathrm{c} / \mathrm{d}$

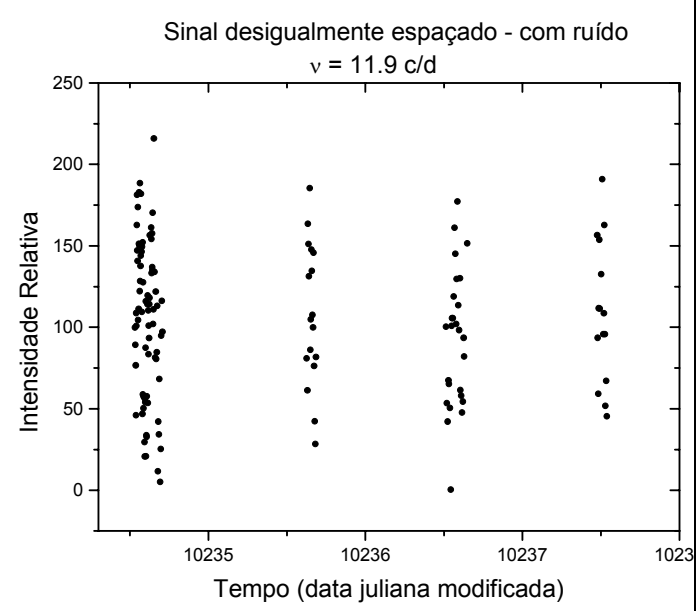

b) sinal de entrada com $11.9 \mathrm{c} / \mathrm{d}$

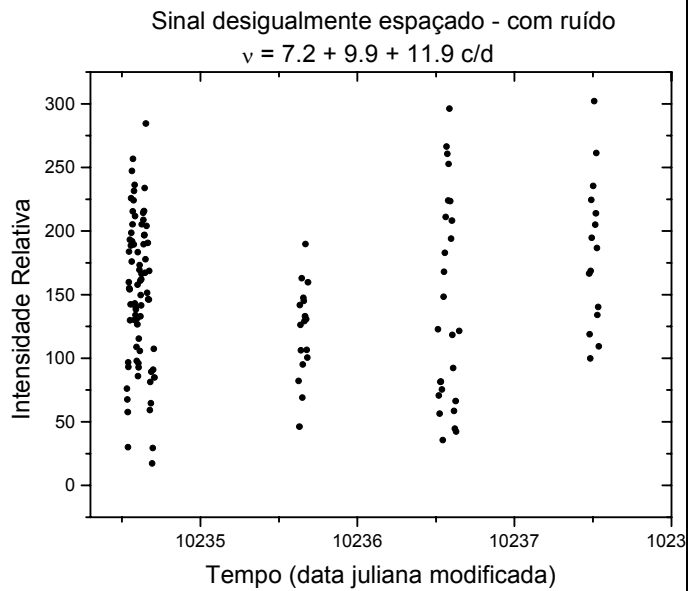

d) sinal de entrada com $7.2+9.9+11.9 \mathrm{c} / \mathrm{d}$

Fig.20. Sinais desigualmente espaçados no tempo, com ruído $\sigma=28$

\begin{tabular}{|c|c|c|}
\hline $\begin{array}{c}\text { Freqüências de } \\
\text { Entrada (ciclos/d) } \\
\text { Freq./(amplitude) }\end{array}$ & $\begin{array}{c}\text { CLEAN } \\
\text { Freq. / (amplitude) }\end{array}$ & $\begin{array}{c}\text { CLEANEST } \\
\text { Freq. / (amplitude) }\end{array}$ \\
\hline $7.2 /(50)$ & $7.1900 /(56)$ & $7.1900 /(50)$ \\
\hline $11.9 /(50)$ & $11.9100 /(52)$ & $11.9000 /(49)$ \\
\hline $7.2+11.9$ & $7.2300 /(51)$ & $7.2200 /(49)$ \\
$(50+50)$ & $12.9200 /(39)$ & $12.9100 /(45)$ \\
\hline $7.2+9.9+11.9$ & $11.9100 /(65)$ & $11.9000 /(60)$ \\
$(50+50+50)$ & $6.1700 /(47)$ & $6.1500 /(51)$ \\
& $8.9700 /(30)$ & $10.9000 /(51)$ \\
\hline
\end{tabular}

Tab. 11. Testes com ruído $\sigma=28$ em dados desigualmente espaçados no tempo. 


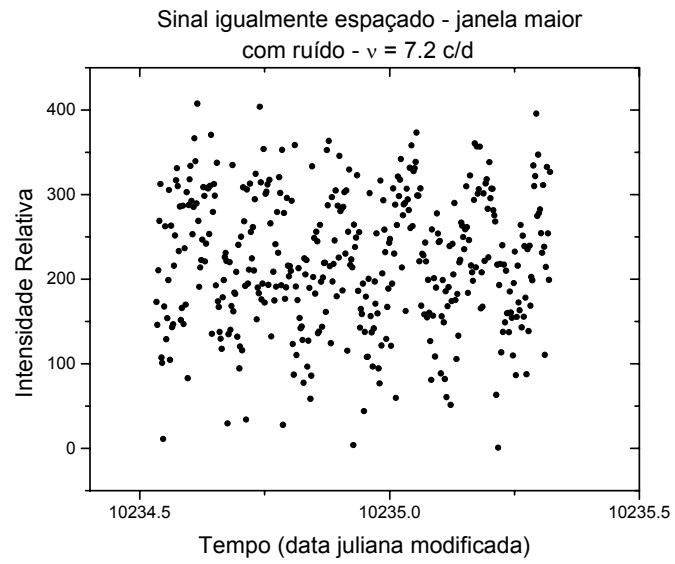

a) sinal de entrada $\mathrm{c} / 7.2 \mathrm{c} / \mathrm{d}$

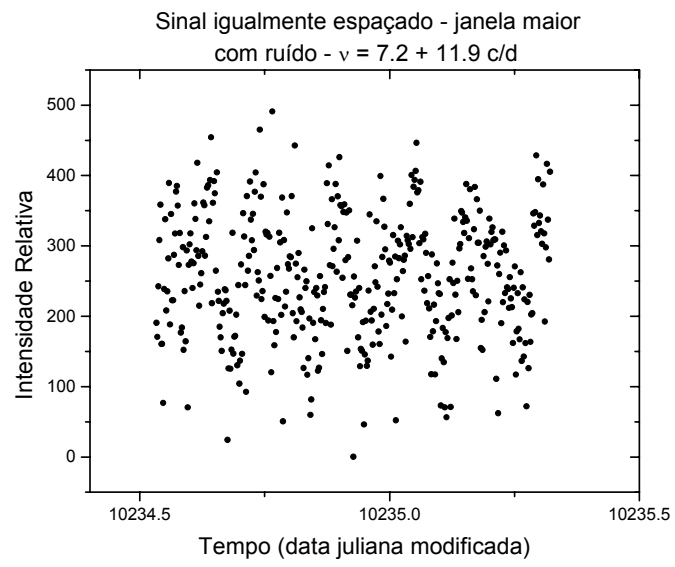

c) sinal de entrada c/ $7.2+11.9 \mathrm{c} / \mathrm{d}$

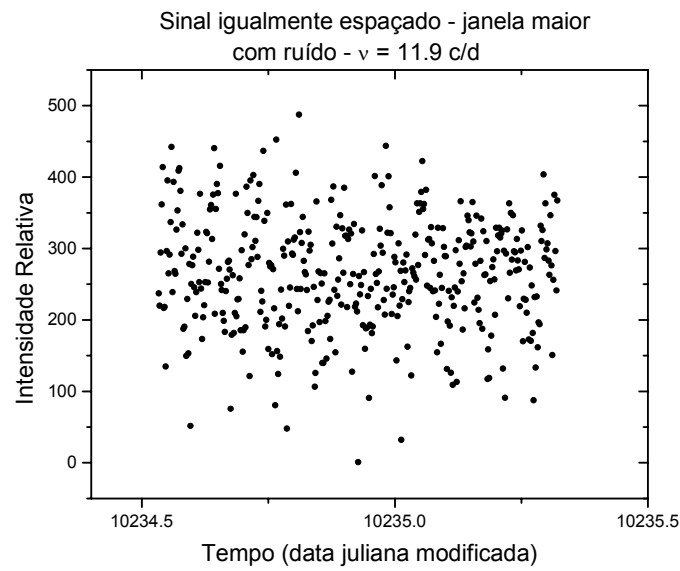

b) sinal de entrada $\mathrm{c} / 11.9 \mathrm{c} / \mathrm{d}$

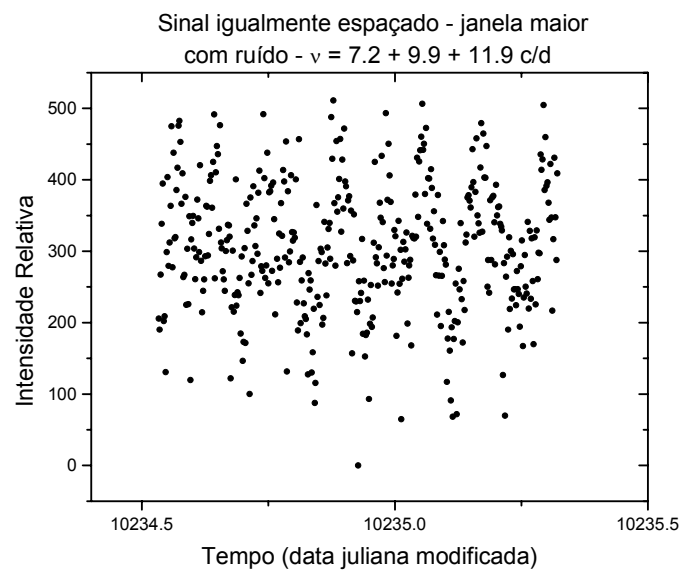

d) sinal de entrada c/ $7.2+9.9+11.9 \mathrm{c} / \mathrm{d}$

Fig.21. Sinais igualmente espaçados no tempo, janela maior, com ruído $\sigma=70$

\begin{tabular}{|c|c|c|}
\hline $\begin{array}{c}\text { Freqüências de } \\
\text { Entrada (ciclos/d) } \\
\text { Freq./(amplitude) }\end{array}$ & $\begin{array}{c}\text { Janela maior } \\
\text { CLEAN } \\
\text { Freq. / (amplitude) }\end{array}$ & $\begin{array}{c}\text { Janela maior } \\
\text { CLEANEST } \\
\text { Freq. / (amplitude) }\end{array}$ \\
\hline $7.2 /(50)$ & $7.1500 /(60)$ & $7.1400 /(56)$ \\
\hline $11.9 /(50)$ & $11.9500 /(55)$ & $11.9400 /(52)$ \\
\hline $7.2+11.9$ & $7.1400 /(58)$ & $7.1400 /(55)$ \\
$(50+50)$ & $11.9600 /(60)$ & $11.9500 /(51)$ \\
\hline $7.2+9.9+11.9$ & $7.1800 /(57)$ & $7.1800 /(55)$ \\
$(50+50+50)$ & $9.8800 /(59)$ & $9.9100 /(51)$ \\
& $12.0000 /(60)$ & $11.9700 /(52)$ \\
\hline
\end{tabular}

Tab. 12. Testes com ruído $\sigma=70$ em dados igualmente espaçados no tempo. 


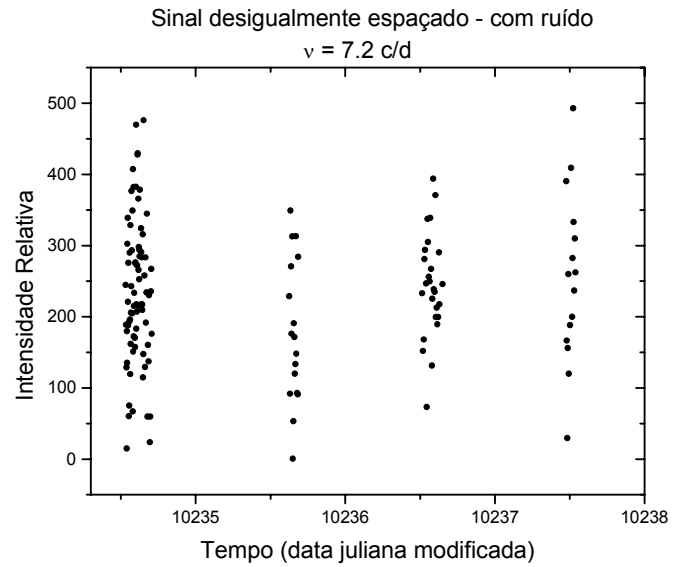

a) sinal de entrada $\mathrm{c} / 7.2 \mathrm{c} / \mathrm{d}$

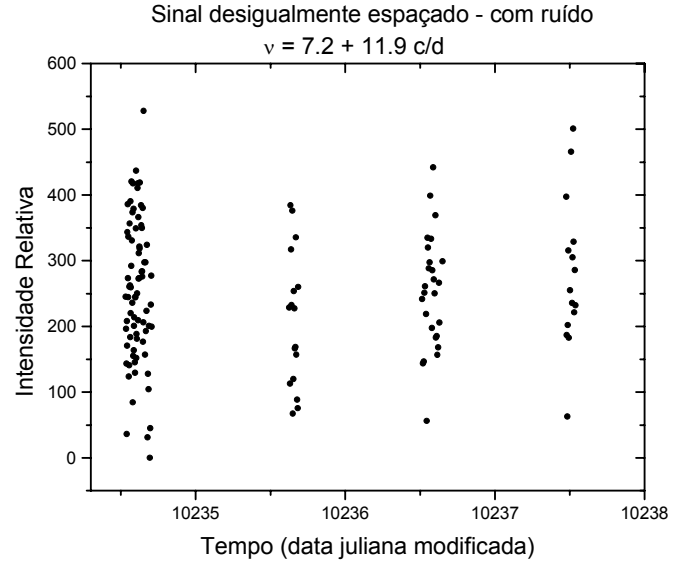

c) sinal de entrada c/ $7.2+11.9 \mathrm{c} / \mathrm{d}$

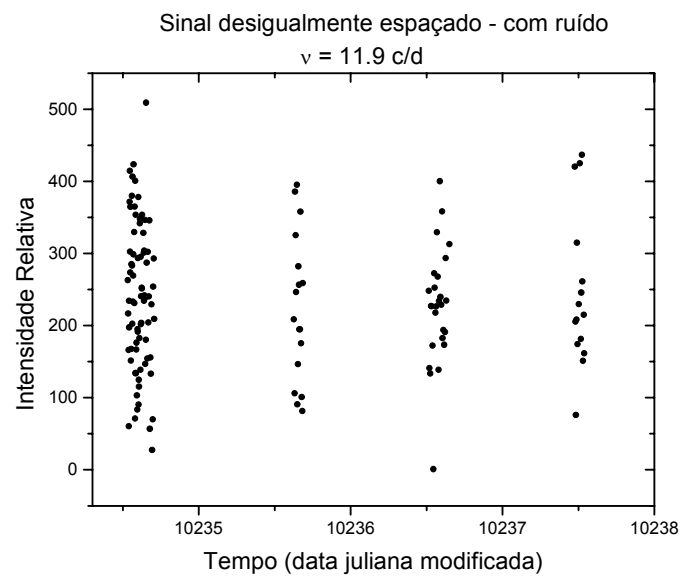

b) sinal de entrada c/ $11.9 \mathrm{c} / \mathrm{d}$

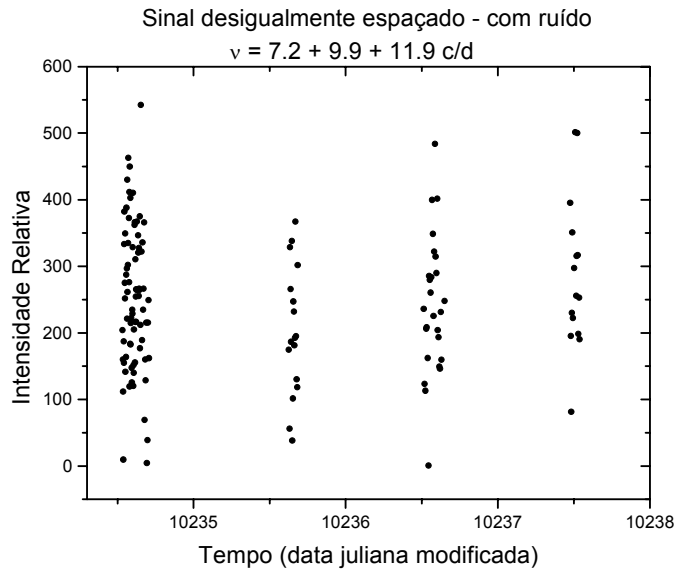

d) sinal de entrada c/ $7.2+9.9+11.9 \mathrm{c} / \mathrm{d}$

Fig.22. Sinais desigualmente espaçados no tempo, com ruído $\sigma=87$

\begin{tabular}{|c|c|c|}
\hline $\begin{array}{c}\text { Freqüências de } \\
\text { Entrada (ciclos/d) } \\
\text { Freq./(amplitude) }\end{array}$ & $\begin{array}{c}\text { Janela maior } \\
\text { CLEAN } \\
\text { Freq. / (amplitude) }\end{array}$ & $\begin{array}{c}\text { Janela maior } \\
\text { CLEANEST } \\
\text { Freq. / (amplitude) }\end{array}$ \\
\hline $7.2 /(50)$ & $6.2000 /(64)$ & $6.2000 /(51)$ \\
\hline $11.9 /(50)$ & $12.9000 /(53)$ & $11.9000 /(46)$ \\
\hline $7.2+11.9$ & $6.1000 /(58)$ & $4.1000 /(62)$ \\
$(50+50)$ & $12.9000 /(56)$ & $11.9000 /(50)$ \\
\hline $7.2+9.9+11.9$ & $11.4000 /(64)$ & $11.9000 /(56)$ \\
$(50+50+50)$ & & \\
\hline
\end{tabular}

Tab. 13. Testes com ruído $\sigma=87$ em dados desigualmente espaçados no tempo. 
É interessante notar a diferença entre os resultados obtidos variando a extensão da janela de observações, no caso de dados igualmente espaçados. Ao variar a extensão da janela, estamos variando o número de ondas que são observadas, de modo que quanto maior o número de ondas, melhor a determinação das freqüências e suas amplitudes.

No caso mais complicado entre todos os expostos anteriormente, onde os dados são desigualmente espaçados e com ruído com desvio padrão $\sigma=87.2$ (o maior ruído usado até agora), com as freqüências injetadas $7.2+9.9+11.9 \mathrm{c} / \mathrm{d}$, ambos os algoritmos encontram apenas uma freqüência, entretanto o resultado do CLEANEST é mais satisfatório tanto em relação à freqüência quanto em relação à sua amplitude. Em geral os dois métodos encontram resultados semelhantes, em alguns casos os dois algoritmos falham na determinação da freqüência principal e detectam seus aliases de $1 \mathrm{c} / \mathrm{d}$, que é uma freqüência presente na janela de observação (periodicidade dia/noite). De uma forma geral o CLEANEST encontrou valores de freqüências e amplitudes mais próximos dos valores de entrada do que o CLEAN.

Até este ponto, os ruídos introduzidos nos sinais periódicos foram constituídos de números aleatórios, sendo todos gerados segundo uma distribuição gaussiana comum, com média nula e desvio padrão $\sigma$. Agora, no caso 2, vamos introduzir um ruído onde cada ponto aleatório apresenta uma distribuição distinta, isto é, cada ponto possui uma variância diferente. A janela de observação será a mesma usada anteriormente, no caso de dados desigualmente espaçados no tempo.

A análise incluindo variação nas amplitudes dos sinais de entrada é mais interessante do que no caso em que as amplitudes são constantes, sendo esse caso mais próximo do encontrado em dados reais. Para simplificar os testes, a proporção entre as amplitudes dos sinais de entrada é sempre um número inteiro. Todos os testes são realizados para o caso em que são injetadas as freqüências $7.2 \mathrm{c} / \mathrm{d}, 9.9 \mathrm{c} / \mathrm{d}$ e $11.9 \mathrm{c} / \mathrm{d}$. A janela de observação utilizada corresponde a uma janela real e o ruído introduzido é constituído de pontos aleatórios, cada número possuindo uma distribuição gaussiana diferente de modo que as variâncias para cada ponto também são diferentes. $O$ fato de cada medida na série temporal possuir peso estatístico diferente é uma característica peculiar de dados astronômicos reais, pois cada ponto da série pertence a um conjunto de mesmo comprimento de onda do interior da linha espectral em estudo, onde cada espectro é tomado num instante de tempo diferente. Desse modo, a relação sinal/ruído para cada espectro dificilmente será a mesma, uma vez que as condições climáticas variam a todo instante (por exemplo a passagem de nuvens, o que diminui o fluxo detectado). 


\section{Caso 2 - Variação nas Amplitudes das Senóides}

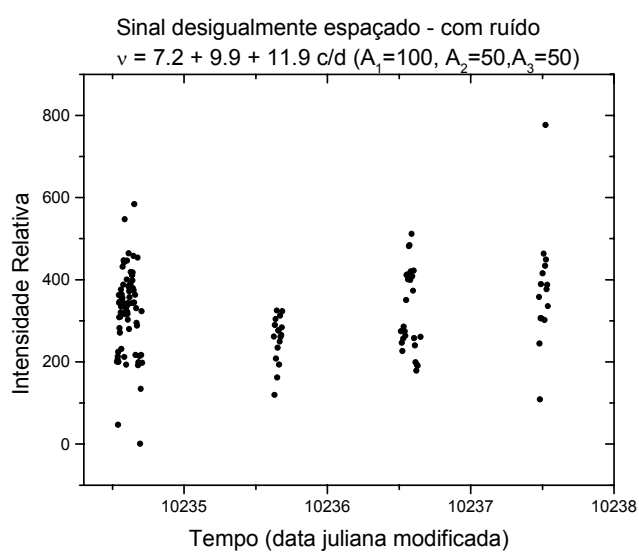

Fig 23 sinal de entrada $\mathrm{c} / \mathrm{A}_{1}=100, \mathrm{~A}_{2}=50, \mathrm{~A}_{3}=50$

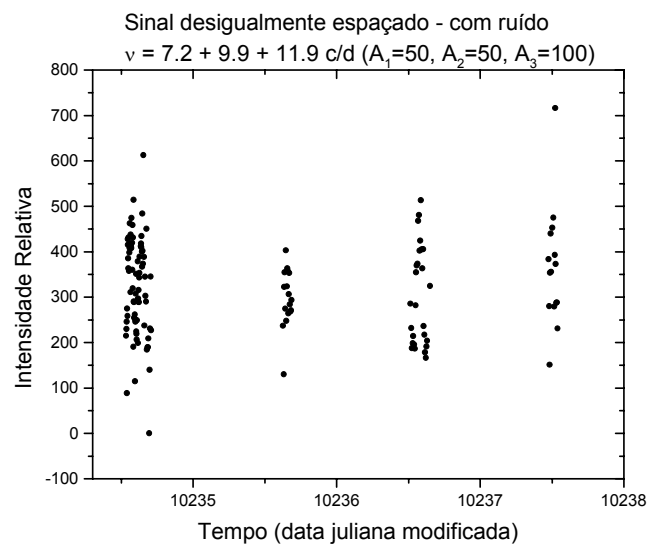

Fig. 25 sinal de entrada $c / A_{1}=50, A_{2}=50, A_{3}=100$

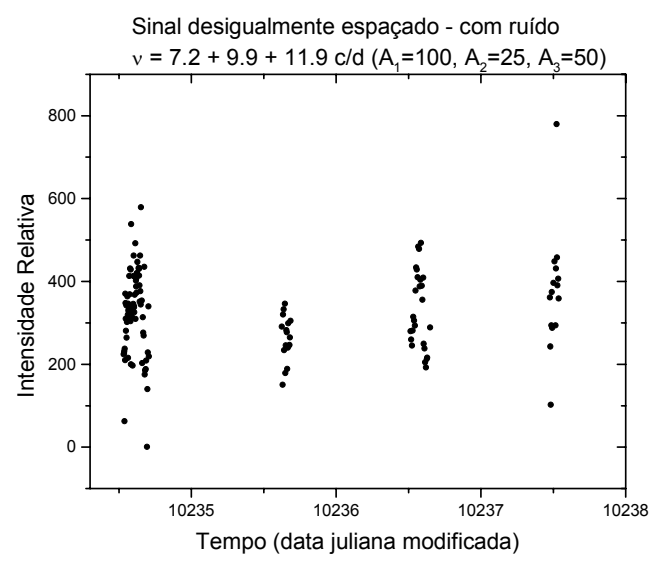

Fig. 27 sinal de entrada $c / A_{1}=100, A_{2}=25, A_{3}=50$

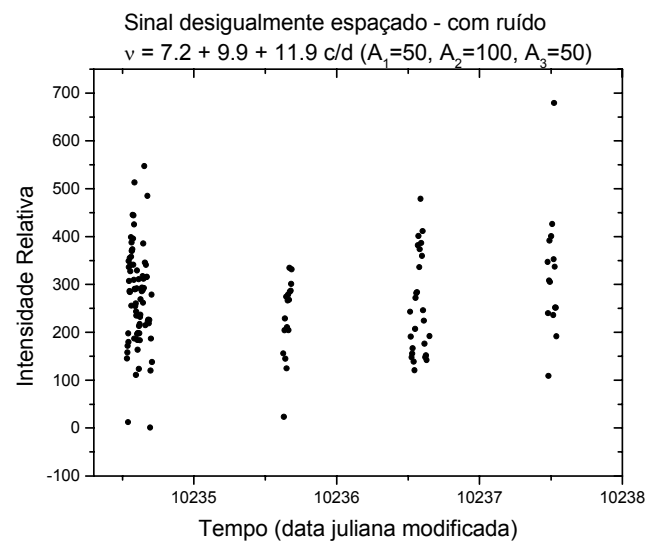

Fig. 24 sinal de entrada $c / A_{1}=50, A_{2}=100, A_{3}=50$

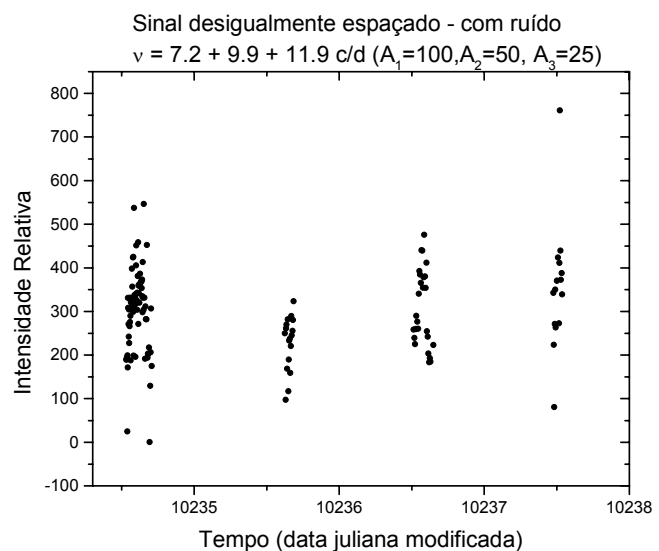

Fig. 26 sinal de entrada $c / A_{1}=100, A_{2}=50, A_{3}=25$

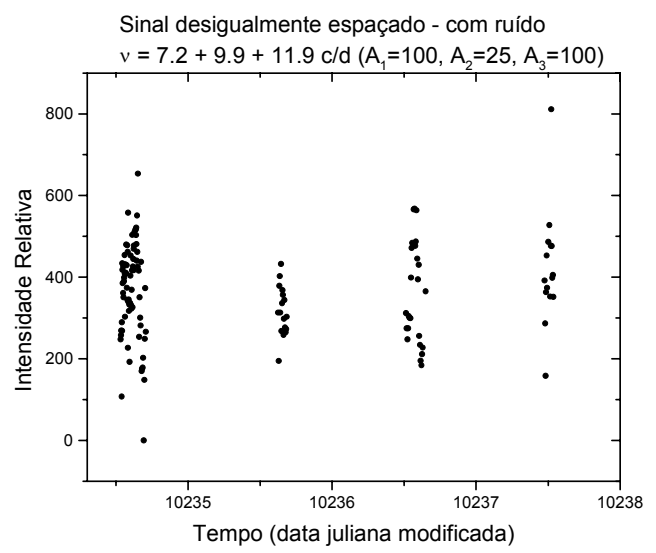

Fig. 28 sinal de entrada $c / A_{1}=100, A_{2}=25$,

$$
\mathrm{A}_{3}=100
$$




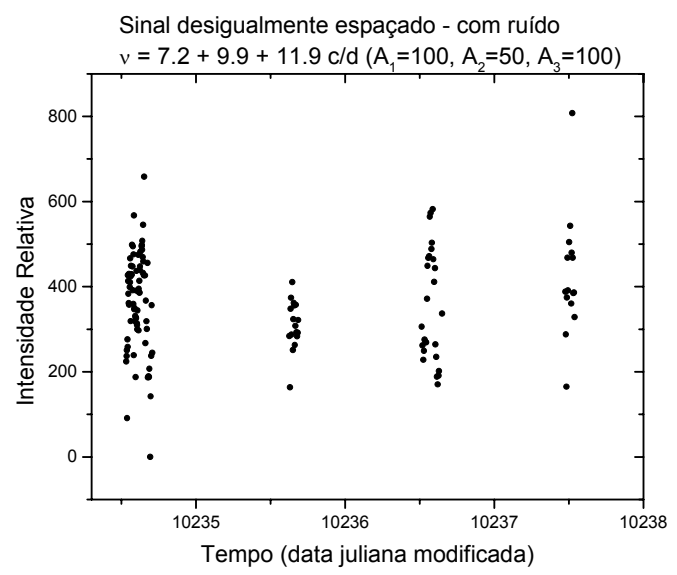

Fig. 29 sinal de entrada $\mathrm{c} / \mathrm{A}_{1}=100, \mathrm{~A}_{2}=50$,

$$
\mathrm{A}_{3}=100
$$

Caso 1:

\begin{tabular}{|c|c|c|}
\hline $\begin{array}{c}\text { Freqüências de } \\
\text { Entrada (ciclos/d) } \\
\text { Freq./(amplitude) }\end{array}$ & $\begin{array}{c}\text { CLEAN } \\
\text { Freq. / (amplitude) }\end{array}$ & $\begin{array}{c}\text { CLEANEST } \\
\text { Freq. / (amplitude) }\end{array}$ \\
\hline $\begin{array}{c}7.2 /(100) \\
+\end{array}$ & $6.1900 /(75)$ & $6.5200 /(45)$ \\
$9.9 /(50)$ & $11.4500 /(50)$ & $11.4500 /(66)$ \\
+ & & \\
$11.9 /(50)$ & & \\
\hline
\end{tabular}

Tab. 14. Testes com os sinais de entrada da Figura 23.

No caso 1 acima, ambos os métodos encontram aproximadamente as mesmas freqüências. A freqüência de $\sim 6.2 \mathrm{c} / \mathrm{d}$ encontrada pelo $C L E A N$ pode ser um alias de $1 \mathrm{c} / \mathrm{d}$ da freqüência principal injetada. É interessante notar aqui que os métodos não concordam muito com relação à amplitude determinada.

\section{Caso 2:}

\begin{tabular}{|c|c|c|}
\hline $\begin{array}{c}\text { Freqüências de } \\
\text { Entrada (ciclos/d) } \\
\text { Freq./(amplitude) }\end{array}$ & $\begin{array}{c}\text { CLEAN } \\
\text { Freq. / (amplitude) }\end{array}$ & $\begin{array}{c}\text { CLEANEST } \\
\text { Freq. / (amplitude) }\end{array}$ \\
\hline $\begin{array}{c}7.2 /(50) \\
+\end{array}$ & $6.1900 /(29)$ & $7.1900 /(62)$ \\
$9.9 /(100)$ & $10.9200 /(80)$ & $9.8500 /(92)$ \\
+ & $11.4500 /(34)$ & $10.9200 /(74)$ \\
$11.9 /(50)$ &
\end{tabular}

Tab. 15. Testes com os sinais de entrada da Figura 24. 
No caso 2, o CLEANEST consegue detectar a freqüência principal (9.9 c/d) e sua amplitude, além da freqüência de $7.2 \mathrm{c} / \mathrm{d}$ e um alias de $1 \mathrm{c} / \mathrm{d}$ da freqüência de $11.9 \mathrm{c} / \mathrm{d}$. A freqüência principal encontrada pelo CLEAN pode ser um alias de $1 \mathrm{c} / \mathrm{d}$ da freqüência original de $9.9 \mathrm{c} / \mathrm{d}$, além de detectar uma freqüência próxima a 11.9, embora com amplitude muito menor que a amplitude de entrada.

Caso 3:

\begin{tabular}{|c|c|c|}
\hline $\begin{array}{c}\text { Freqüências de } \\
\text { Entrada (ciclos/d) } \\
\text { Freq./(amplitude) }\end{array}$ & $\begin{array}{c}\text { CLEAN } \\
\text { Freq. / (amplitude) }\end{array}$ & $\begin{array}{c}\text { CLEANEST } \\
\text { Freq. / (amplitude) }\end{array}$ \\
\hline $\begin{array}{c}7.2 /(50) \\
+\end{array}$ & $6.1900 /(31)$ & $9.2500 /(43)$ \\
$9.9 /(50)$ & $10.3800 /(29)$ & $11.9200 /(112)$ \\
+ & $11.9160 /(84)$ & \\
$11.9 /(100)$ & & \\
\hline
\end{tabular}

Tab. 16. Testes com os sinais de entrada da Figura 25.

No caso 3, ambos os métodos encontram a freqüência principal, embora a amplitude da freqüência detectada pelo CLEANEST seja mais próxima da injetada. Além disso, ele determina uma freqüência próxima à de $9.9 \mathrm{c} / \mathrm{d}$ de entrada, entretanto falha em determinar a freqüência $7.2 \mathrm{c} / \mathrm{d}$, enquanto que o CLEAN encontra o alias de $1 \mathrm{c} / \mathrm{d}$ dessa freqüência.

\section{Caso 4:}

\begin{tabular}{|c|c|c|}
\hline $\begin{array}{c}\text { Freqüências de } \\
\text { Entrada (ciclos/d) } \\
\text { Freq./(amplitude) }\end{array}$ & $\begin{array}{c}C L E A N \\
\text { Freq. / (amplitude) }\end{array}$ & $\begin{array}{c}\text { CLEANEST } \\
\text { Freq. / (amplitude) }\end{array}$ \\
\hline $\begin{array}{c}7.2 /(100) \\
+\end{array}$ & $6.1900 /(73)$ & $7.1900 /(101)$ \\
$9.9 /(50)$ & $12.5100 /(44)$ & $10.8500 /(59)$ \\
+ & & \\
$11.9 /(25)$ & & \\
\hline
\end{tabular}

Tab. 17. Testes com os sinais de entrada da Figura 26.

Novamente, o CLEANEST determina a freqüência principal, além de um alias de $1 \mathrm{c} / \mathrm{d}$ da segunda freqüência. O CLEAN determina um alias de $1 \mathrm{c} / \mathrm{d}$ da freqüência principal, além de um alias em $2 \mathrm{c} / \mathrm{d}$ da freqüência secundária. 


\section{Caso 5:}

\begin{tabular}{|c|c|c|}
\hline $\begin{array}{c}\text { Freqüências de } \\
\text { Entrada (ciclos/d) } \\
\text { Freq./(amplitude) }\end{array}$ & $\begin{array}{c}\text { CLEAN } \\
\text { Freq. / (amplitude) }\end{array}$ & $\begin{array}{c}\text { CLEANEST } \\
\text { Freq. / (amplitude) }\end{array}$ \\
\hline $\begin{array}{c}7.2 /(100) \\
+\end{array}$ & $6.1900 /(81)$ & $7.1900 /(116)$ \\
$9.9 /(25)$ & $11.4500 /(44)$ & $11.9200 /(56)$ \\
+ & & \\
$11.9 /(50)$ & & \\
\hline
\end{tabular}

Tab. 18. Testes com os sinais de entrada da Figura 27.

No caso 5, as duas freqüências principais são bem determinadas pelo CLEANEST. O método falha em determinar a freqüência de $9.9 \mathrm{c} / \mathrm{d}$. O CLEAN encontra um alias de 2 c/d da freqüência principal e a terceira freqüência.

\section{Caso 6:}

\begin{tabular}{|c|c|c|}
\hline $\begin{array}{c}\text { Freqüências de } \\
\text { Entrada (ciclos/d) } \\
\text { Freq./(amplitude) }\end{array}$ & $\begin{array}{c}\text { CLEAN } \\
\text { Freq. / (amplitude) }\end{array}$ & $\begin{array}{c}\text { CLEANEST } \\
\text { Freq. / (amplitude) }\end{array}$ \\
\hline $\begin{array}{c}7.2 /(100) \\
+\end{array}$ & $6.1900 /(80)$ & $5.1200 /(100)$ \\
$9.9 /(25)$ & $11.8490 /(75)$ & $11.8500 /(93)$ \\
+ & & \\
$11.9 /(100)$ & & \\
\hline
\end{tabular}

Tab. 19. Testes com os sinais de entrada da Figura 28.

No caso 6, o CLEANEST encontra a freqüência $11.9 \mathrm{c} / \mathrm{d}$ e encontra um possível alias da freqüência de $7.2 \mathrm{c} / \mathrm{d}$, falhando em determinar a freqüência de $9.9 \mathrm{c} / \mathrm{d}$.

\section{Caso 7:}

\begin{tabular}{|c|c|c|}
\hline $\begin{array}{c}\text { Freqüências de } \\
\text { Entrada (ciclos/d) } \\
\text { Freq./(amplitude) }\end{array}$ & $\begin{array}{c}\text { CLEAN } \\
\text { Freq. / (amplitude) }\end{array}$ & $\begin{array}{c}\text { CLEANEST } \\
\text { Freq. / (amplitude) }\end{array}$ \\
\hline $\begin{array}{c}7.2 /(100) \\
+\end{array}$ & $6.1900 /(59)$ & $7.1900 /(106)$ \\
$9.9 /(50)$ & $11.9160 /(53)$ & $9.8500 /(74)$ \\
+ & & $12.9100 /(66)$ \\
$11.9 /(100)$ & & \\
\hline
\end{tabular}

Tab. 20. Testes com os sinais de entrada da Figura 29. 
No caso 7, as freqüências de $7.2 \mathrm{c} / \mathrm{d}$ e $9.9 \mathrm{c} / \mathrm{d}$ foram encontradas pelo CLEANEST, que achou ainda um alias de $1 \mathrm{c} / \mathrm{d}$ da freqüência de $11.9 \mathrm{c} / \mathrm{d}$. O método CLEAN encontra um alias de $7.2 \mathrm{c} / \mathrm{d}$ e a freqüência $11.9 \mathrm{c} / \mathrm{d}$.

$\mathrm{Na}$ figura a seguir, são apresentados os resultados finais de todos os testes realizados com ambos os métodos.
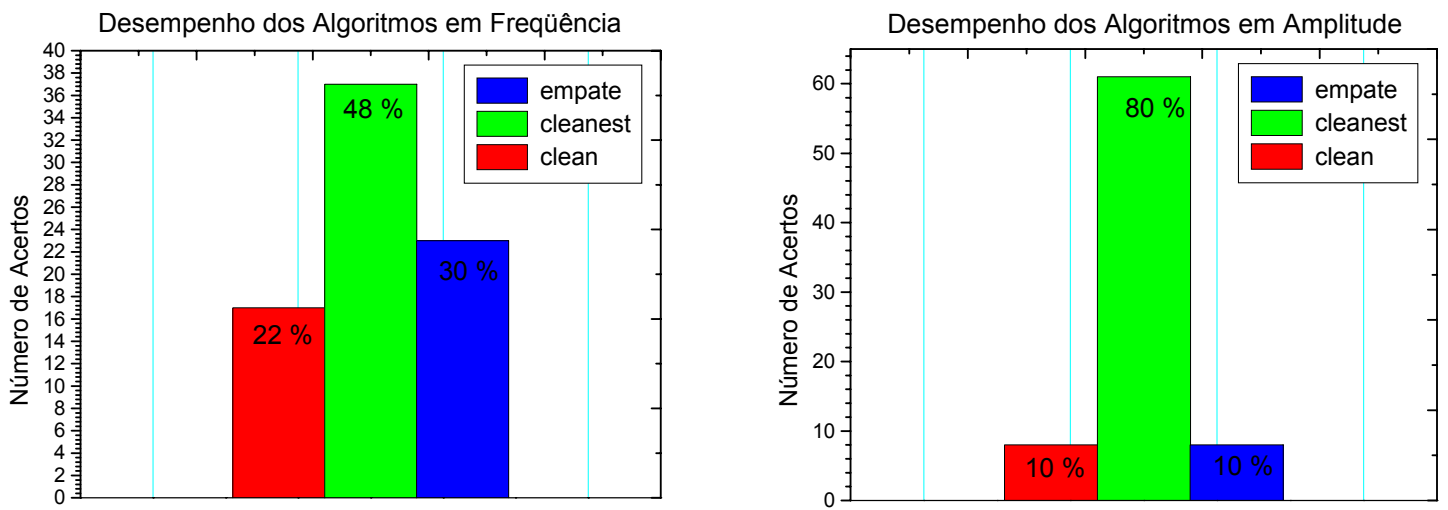

Fig.30. Resultados das análises realizadas com os algoritmos CLEAN e CLEANEST. No histograma à esquerda é mostrado o número de acertos e empates de ambos os algoritmos na determinação das freqüências de entrada, em relação ao número total de freqüências obtidas. No painel à direita, é mostrado o número de acertos e empates na determinação das amplitudes dos sinais.

A comparação do desempenho entre dois métodos de análise temporal é algo complicado de ser feito, uma vez que os resultados obtidos dependem muito da janela de observações. Empiricamente, pode-se constatar que um tipo específico de janela pode favorecer mais a determinação de uma freqüência do que outra.

Nas análises temporais dos espectros da estrela Eta Centauri que se seguem, optei pela utilização do algoritmo CLEANEST uma vez que seus resultados nos testes anteriores foram em média mais bem sucedidos tanto na determinação das freqüências como na determinação de suas amplitudes. Além disso, o tempo de cálculo empregado pelo algoritmo CLEANEST é, em muitos casos, inferior ao tempo de cálculo do CLEAN.

Um fator que pode justificar a diferença no desempenho entre os métodos é o fato de o algoritmo CLEAN assumir que a média do sinal presente nos resíduos a cada iteração é nula. Esse fato pressupõe necessariamente a existência de um número inteiro de ciclos nos dados amostrados, o que dificilmente ocorre na prática. Em contrapartida, o algoritmo CLEANEST calcula a média do sinal a cada iteração. 


\section{ANÁLISE DOS DADOS}

A partir dos espectros centrados em He I $\lambda 667.8 \mathrm{~nm}$, foram criados 5 conjuntos (um conjunto para cada um dos grupos: 1996+1997+1998, 1997+1998, 1996, 1997, 1998) com 241 séries temporais cada, no intervalo de $666.49 \mathrm{~nm}$ até $668.89 \mathrm{~nm}$, sendo uma série para cada comprimento de onda, com passos de $0.01 \mathrm{~nm}$. Em seguida, essas séries foram analisadas utilizando-se o algoritmo CLEANEST com passos em freqüência de 1/1000 da freqüência mínima a ser detectada. Foram também criadas séries temporais a partir de medidas da Largura Equivalente $(E W)$, Largura a Meia Altura (FWHM) e Velocidade Radial $(R V)$ desses espectros. Com os resultados obtidos nessa análise, foram confeccionados os periodogramas mostrados a seguir, os quais apresentam as freqüências estatísticamente significativas.

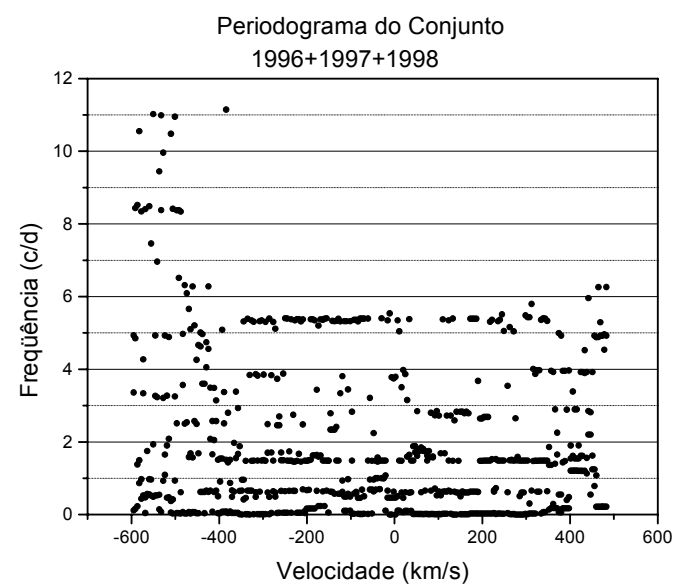

Fig.31. Periodograma dos resultados do CLEANEST para 1996/1997/1998. Pode-se ver sinais com $0.6 \mathrm{c} / \mathrm{d}, 1.48 \mathrm{c} / \mathrm{d}$ e $5.3 \mathrm{c} / \mathrm{d}$.

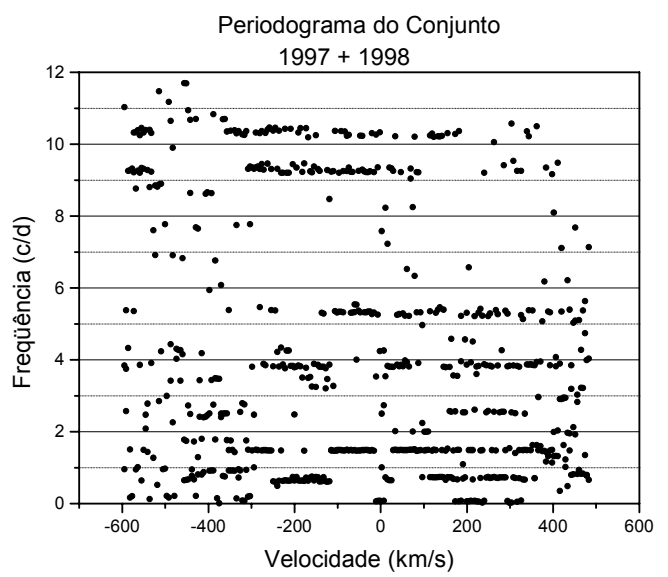

Fig.32. Periodograma dos resultados do CLEANEST para os anos 1997/1998. Podem ser observadas as freqüências de $0.6 \mathrm{c} / \mathrm{d}, 1.48 \mathrm{c} / \mathrm{d}, 3.8 \mathrm{c} / \mathrm{d}, 5.3 \mathrm{c} / \mathrm{d}$, $9.2 \mathrm{c} / \mathrm{d}$ e $10.3 \mathrm{c} / \mathrm{d}$. 


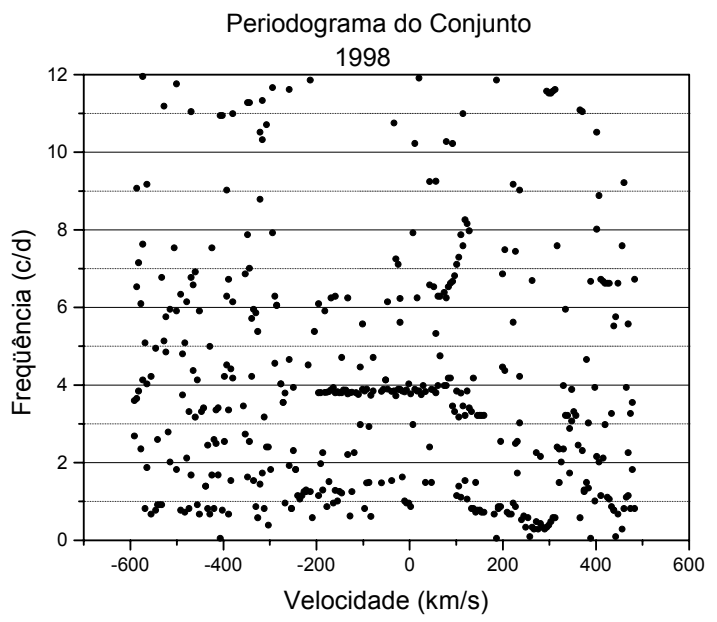

Fig.33. Periodograma dos resultados do CLEANEST para o ano de 1998 mostrando variabilidade em $3.8 \mathrm{c} / \mathrm{d}$.

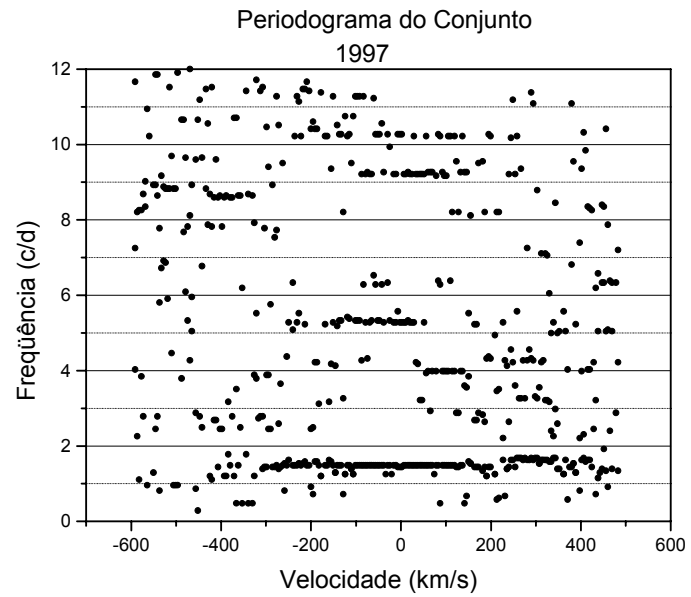

Fig.34. Periodograma dos resultados do CLEANEST para o ano de 1997 mostrando as freqüências de $1.48 \mathrm{c} / \mathrm{d}, 3.8 \mathrm{c} / \mathrm{d}, 5.3 \mathrm{c} / \mathrm{d}, 9.2 \mathrm{c} / \mathrm{d}$ e $10.3 \mathrm{c} / \mathrm{d}$.

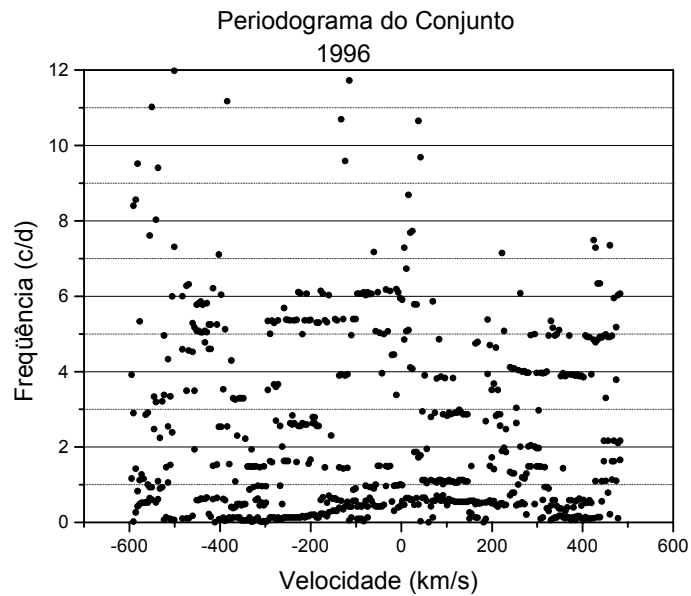

Fig.35. Periodograma dos resultados do CLEANEST para o ano de 1996 mostrando as freqüências de $0.6 \mathrm{c} / \mathrm{d}, 1.48 \mathrm{c} / \mathrm{d}$ (fraca) e $5.3 \mathrm{c} / \mathrm{d}$. 


\subsection{Análise das Variações em $\mathrm{W}_{\lambda}, \mathrm{FWHM}$ e $\mathrm{V}_{\text {radial }}$}

A seguir, temos os gráficos das variabilidades em largura equivalente $\left(\mathrm{W}_{\lambda}\right)$, largura a meia altura (FWHM) e velocidade radial $\left(\mathrm{V}_{\text {radial }}\right)$ para os espectros das missões de 1996, 1997 e 1998.

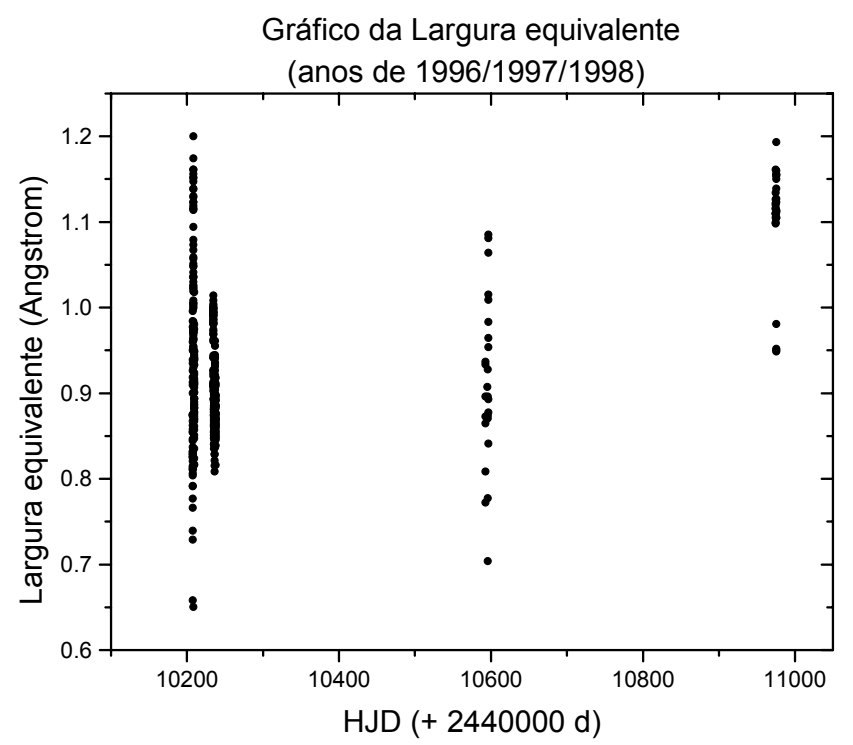

Fig.36. Gráfico da largura equivalente $\left(\mathrm{W}_{\lambda}\right)$ em função do tempo (data juliana modificada) dos espectros dos anos de 1996/1997/1998.

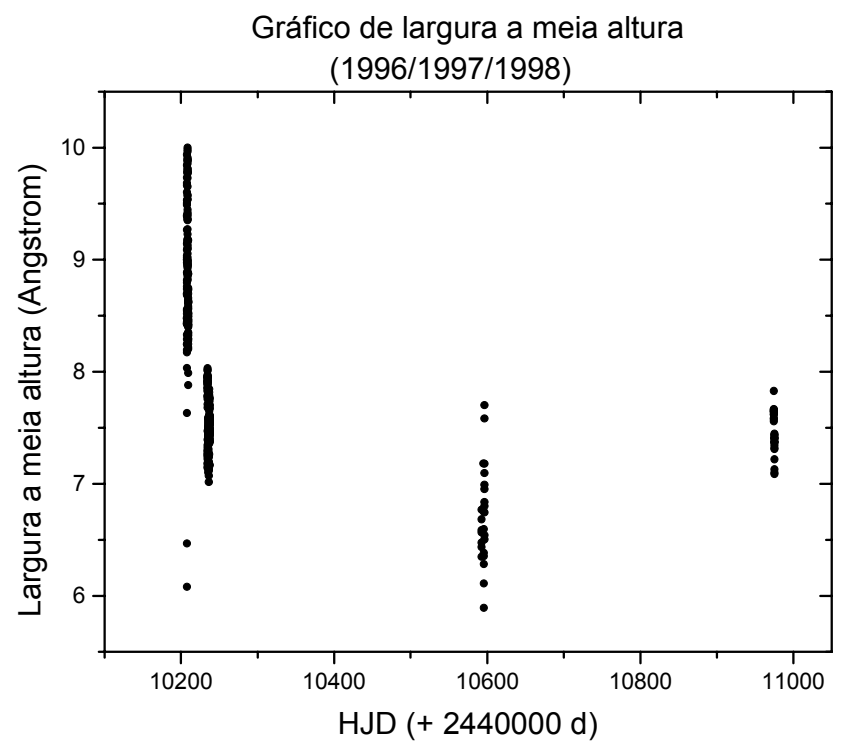

Fig.37. Gráfico da largura a meia altura (FWHM) em função do tempo (data juliana modificada) dos espectros dos anos de 1996/1997/1998. 


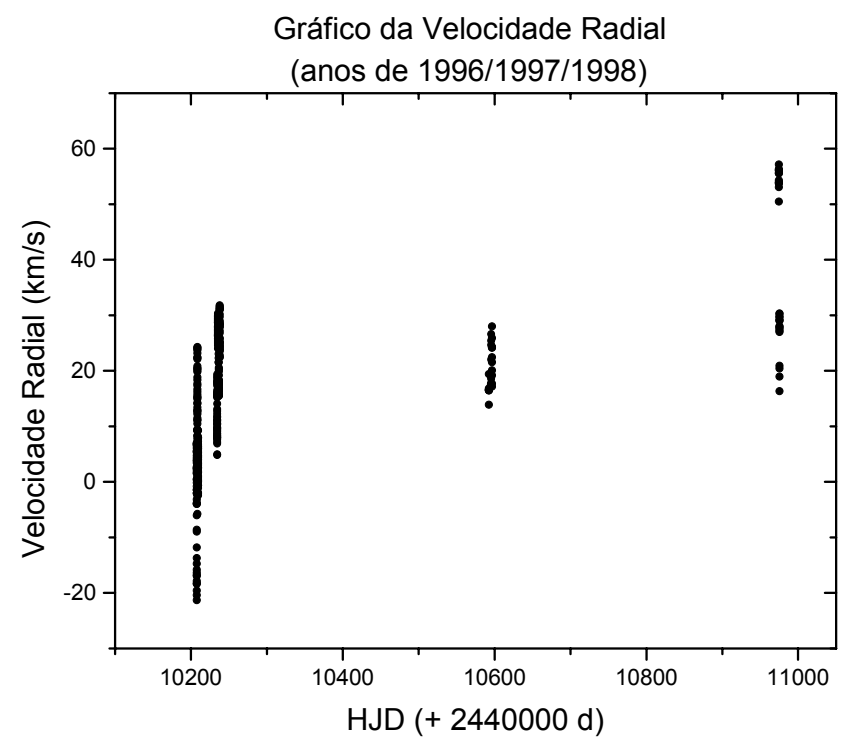

Fig.38. Gráfico da velocidade radial $\left(\mathrm{V}_{\text {radial }}\right)$ em função do tempo (data juliana modificada) dos espectros dos anos de 1996/1997/1998.

\begin{tabular}{cccc}
\hline $\begin{array}{c}\text { Freqüência }(\mathrm{c} / \mathrm{d}) \\
( \pm 0.01)\end{array}$ & $\chi^{2}$ & Significado & $\begin{array}{c}\text { Variabilidades } \\
\text { Observadas }\end{array}$ \\
\hline 1.65 & $\sim 52$ & $99.5 \%$ & $\mathrm{~V}_{\text {radial }}(1996)$ \\
3.58 & $\sim 9$ & $75 \%$ & $\mathrm{~V}_{\text {radial }}(1997 / 98)$ \\
0.73 & $\sim 8.7$ & $75 \%$ & $\mathrm{~W}_{\lambda}(1997 / 98)$ \\
0.55 & $\sim 40$ & $99.5 \%$ & $\mathrm{~W}_{\lambda}(1996)$ \\
0.71 & $\sim 8.6$ & $75 \%$ & $\mathrm{FWHM}(1997 / 98)$ \\
4.99 & $\sim 51$ & $99.5 \%$ & $\mathrm{FWHM}(1996)$ \\
1.48 & $\sim 60$ & $99.5 \%$ & $\mathrm{FWHM}$ \\
1.67 & & & $(1996 / 97 / 98)$ \\
0.66 & $\sim 46$ & $99.5 \%$ & $\mathrm{~W}_{\lambda}(1996 / 97 / 98)$ \\
& $\sim 61$ & $99.5 \%$ & $\mathrm{~V}_{\text {radial }}(1996 / 97 / 98)$ \\
\hline
\end{tabular}

Tab. 21. Resumo das principais freqüências detectadas em velocidade radial, largura equivalente e largura a meia altura ao longo dos anos de 1996, 1997 e 1998. As incertezas nas freqüências correspondem às incertezas na determinação de suas fases.

Podemos agora resumir os resultados obtidos através do estudo dos perfis de linha ( $l p v, \mathrm{~W}_{\lambda}$, FWHM e $\left.\mathrm{V}_{\text {radial }}\right)$ na tabela seguinte: 


\begin{tabular}{|c|c|c|c|}
\hline Freqüência (c/d) & $\chi^{2}$ & Significado & Variabilidades Observadas \\
\hline $0.61 \pm 0.05$ & $\sim 70$ & $99.5 \%$ & $\begin{array}{ll}\text { - } & l p v \text { em } \lambda 667.8 \mathrm{~nm}(1996-1998) \\
- & \text { compatível com } 0.55 \mathrm{c} / \mathrm{d} \text { em } \mathrm{W}_{\lambda} \\
& (1996) \\
\text { - } & \text { próxima de } 0.71 \mathrm{c} / \mathrm{d} \text { em } \mathrm{FWHM} \\
& (1997+1998) \\
- & \text { próxima de } 0.73 \mathrm{c} / \mathrm{d} \text { em } \mathrm{W}_{\lambda} \\
& (1997+1998) \\
- & \text { compatível com 0.66 c/d em } \\
& \mathrm{V}_{\text {radial }}(1996-1998) \\
\text { - } & \text { próxima de } 0.78 \mathrm{c} / \mathrm{d} \text { em Janot et } \\
\text { al. }(1999)\end{array}$ \\
\hline $1.48 \pm 0.05$ & $\sim 70$ & $99.5 \%$ & $\begin{array}{ll}\text { - } & \text { Compatível com 1.48 c/d em } \\
& \text { FWHM (1996-1998) } \\
\text { - } & \text { Compatível com 1.65 c/d em } \\
& \mathrm{V}_{\text {radial }}(1996) \\
\text { - } & \text { lpv em } \lambda 667.8 \mathrm{~nm}(1996-1998) \\
\text { - } & \text { compatível com } 1.67 \mathrm{c} / \mathrm{d} \text { em } \mathrm{W}_{\lambda} \\
& (1996-1998) \\
\text { - } & \text { detectada por Balona et al. } \\
& \text { (1999) } \\
\text { - } & \text { detectada por Janot et al.(1999) }\end{array}$ \\
\hline $3.81 \pm 0.28$ & $\sim 8$ & $75 \%$ & $\begin{array}{ll}\text { - } & l p v \text { em } \lambda 667.8 \mathrm{~nm}(1997+1998) \\
\text { - } & \text { detectada por Janot } \text { et al. }(1999) \\
\text { - } & \text { próxima de } 3.58 \mathrm{c} / \mathrm{d} \text { em } \mathrm{V}_{\text {radial }} \\
& (1997 / 98)\end{array}$ \\
\hline $5.31 \pm 0.19$ & $\sim 60$ & $99.5 \%$ & $\begin{array}{ll}- & l p v \text { em } \lambda 667.8 \mathrm{~nm}(1996-1998) \\
- & \text { próxima de } 4.99 \mathrm{c} / \mathrm{d} \text { em FWHM } \\
& (1996)\end{array}$ \\
\hline $9.24 \pm 0.19$ & $\sim 8$ & $75 \%$ & - $\quad l p v$ em $\lambda 667.8 \mathrm{~nm}(1997+1998)$ \\
\hline $10.35 \pm 0.13$ & $\sim 8$ & $75 \%$ & - $\quad l p v$ em $\lambda 667.8 \mathrm{~nm}(1997+1998)$ \\
\hline
\end{tabular}

Tab. 22. Resumo das principais freqüências detectadas e as variabilidades observadas. As pesquisas com o CLEANEST para cada série temporal foram feitas buscando 3 freqüências, então o número de graus de liberdade será 3 × $2=6$. Consultando-se uma tabela de valores de Qui-Quadrado, obtemos os valores de significância mostrados. 
Os valores das freqüências na Tabela 22 correspondem às médias dos valores encontrados nos periodogramas anteriores. Assim, os valores de $0.61,1.48$ e $5.31 \mathrm{c} / \mathrm{d}$ são os determinados a partir do conjunto de dados de 1996/1997/1998, enquanto que as freqüências de 3.81, 9.24 e 10.35 c/d correspondem ao conjunto de 1997/1998. O grau de confiabilidade das freqüências obtidas no conjunto 1996/1997/1998 é maior do que no conjunto de 1997/1998, uma vez que o número de pontos nas séries temporais é bem maior. Esse fato se torna mais explícito através dos valores de $\chi^{2}$ mostrados na tabela. As incertezas nas freqüências da tabela correspondem às incertezas das médias das freqüências mostradas nos periodogramas. É comum encontrar-se na literatura alguns valores diferentes de freqüencias, uma vez que os modos de pulsação não são estáveis. No trabalho de Janot et al (1999) foram analisadas séries temporais de anos anteriores (1993 e 1995) e encontradas algumas freqüências que não aparecem neste trabalho, como sinais em $0.78 \mathrm{c} / \mathrm{d}, 1.29 \mathrm{c} / \mathrm{d}, 1.78$ $\mathrm{c} / \mathrm{d}$ e $4.51 \mathrm{c} / \mathrm{d}$. Outras, como $1.48 \mathrm{c} / \mathrm{d}$ e $3.82 \mathrm{c} / \mathrm{d}$, aparecem nesse artigo e foram detectadas aqui também. Esse fato pode ser um indício de que essas ondas interferem construtiva e destrutivamente. 
A seguir são mostrados os diagramas em escala de cinza dos resíduos médios dos espectros analisados. Para maior clareza, os resíduos foram exibidos em fase (em unidades de $2 \pi$ radianos) com as freqüências acima determinadas, onde as regiões mais claras apresentam maior intensidade que as regiões escuras.

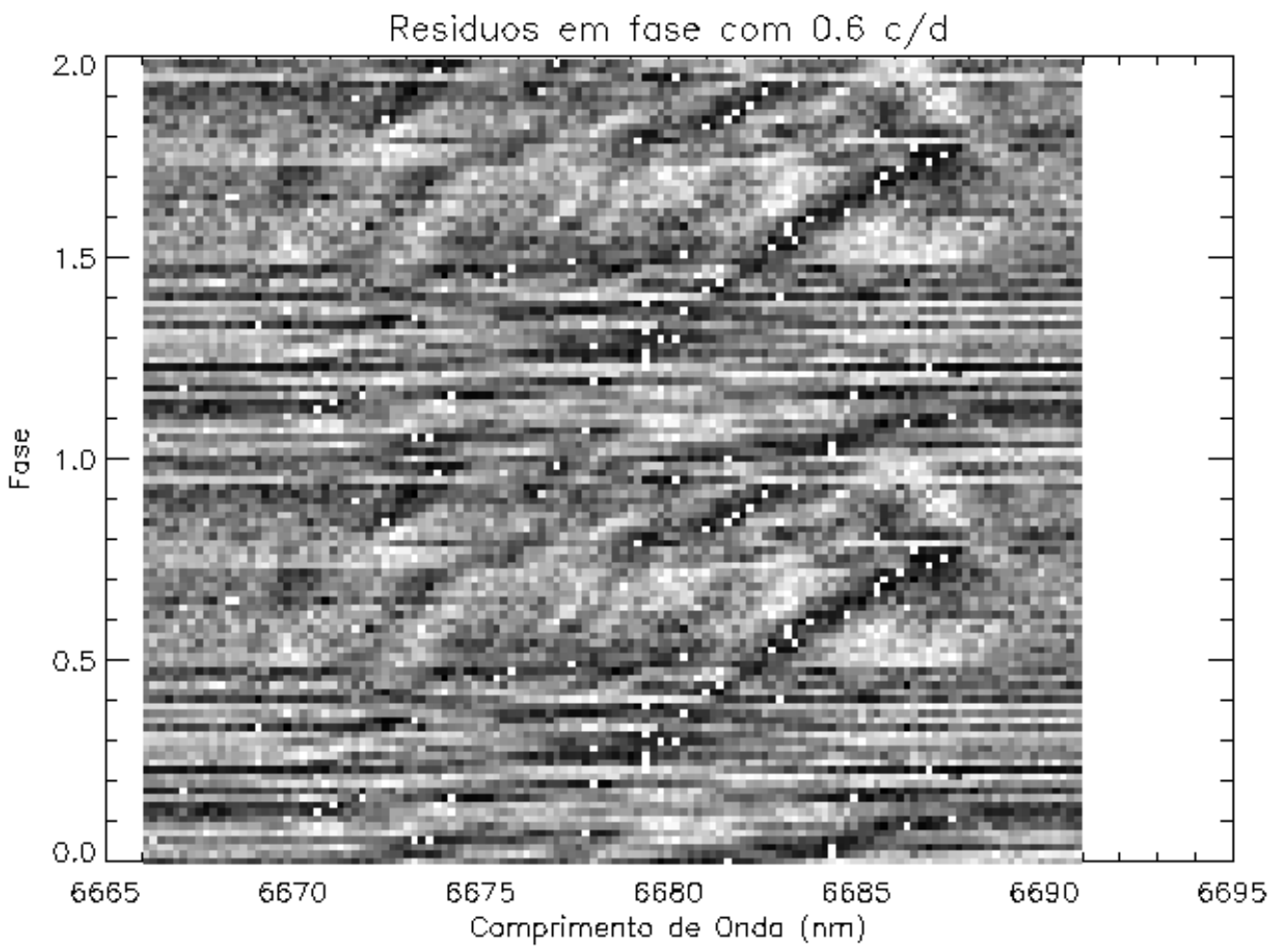

Fig.39. Diagrama em escala de cinza mostrando os resíduos médios dos espectros centrados em $\lambda 667.8 \mathrm{~nm}$. Note a presença de regiões claras dispostas em alternância com regiões mais escuras que se propagam ao longo do perfil de linha. Para maior clareza, os resíduos foram mostrados em fase com a freqüência de 0.6 ciclos/dia e dois ciclos inteiros de oscilação ( 0 a $4 \pi$ radianos). 


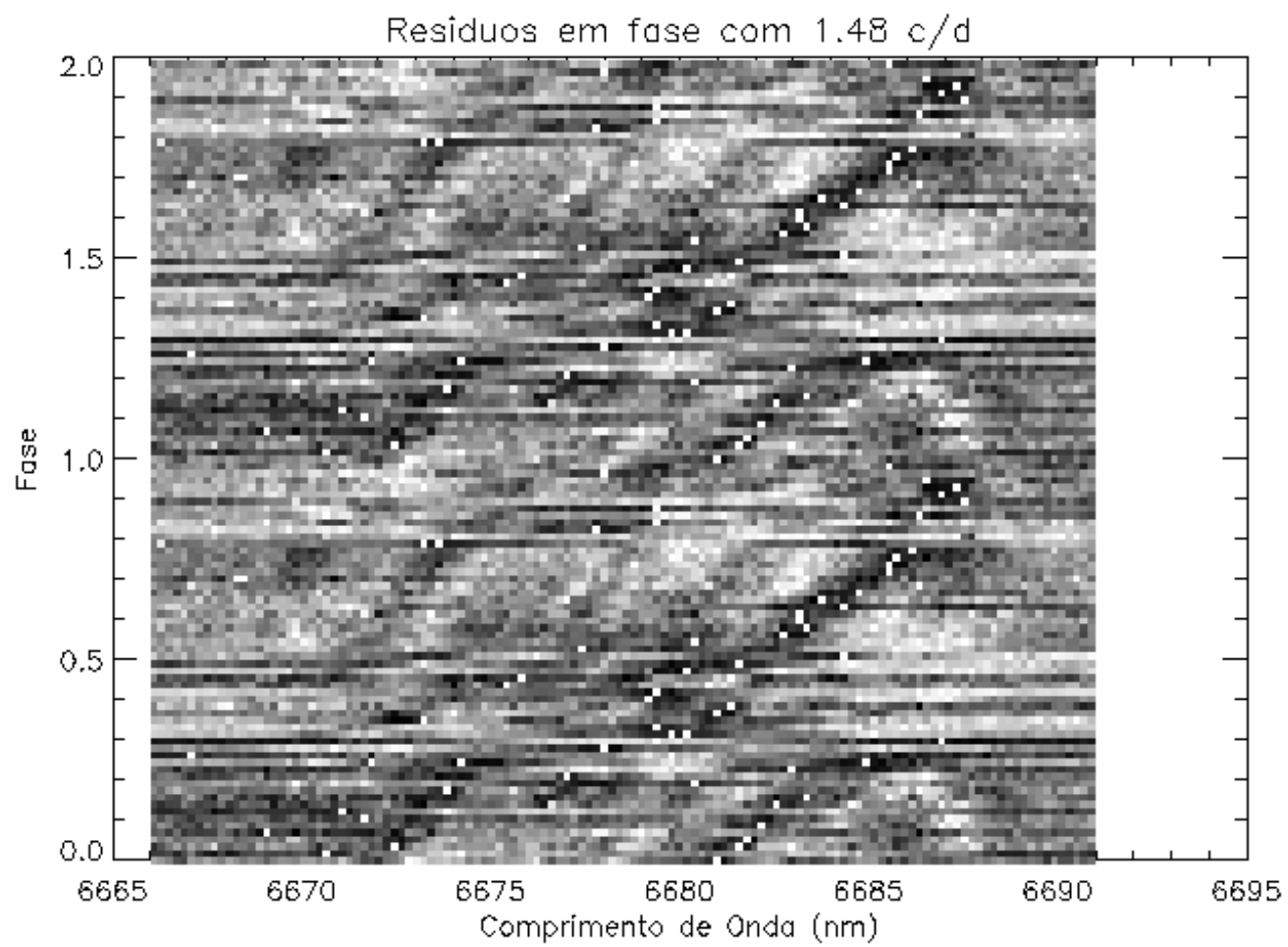

Fig.40. Diagrama em escala de cinza mostrando os resíduos médios dos espectros centrados em $\lambda 667.8 \mathrm{~nm}$. Os resíduos foram mostrados em fase com a freqüência de 1.48 ciclos/dia.

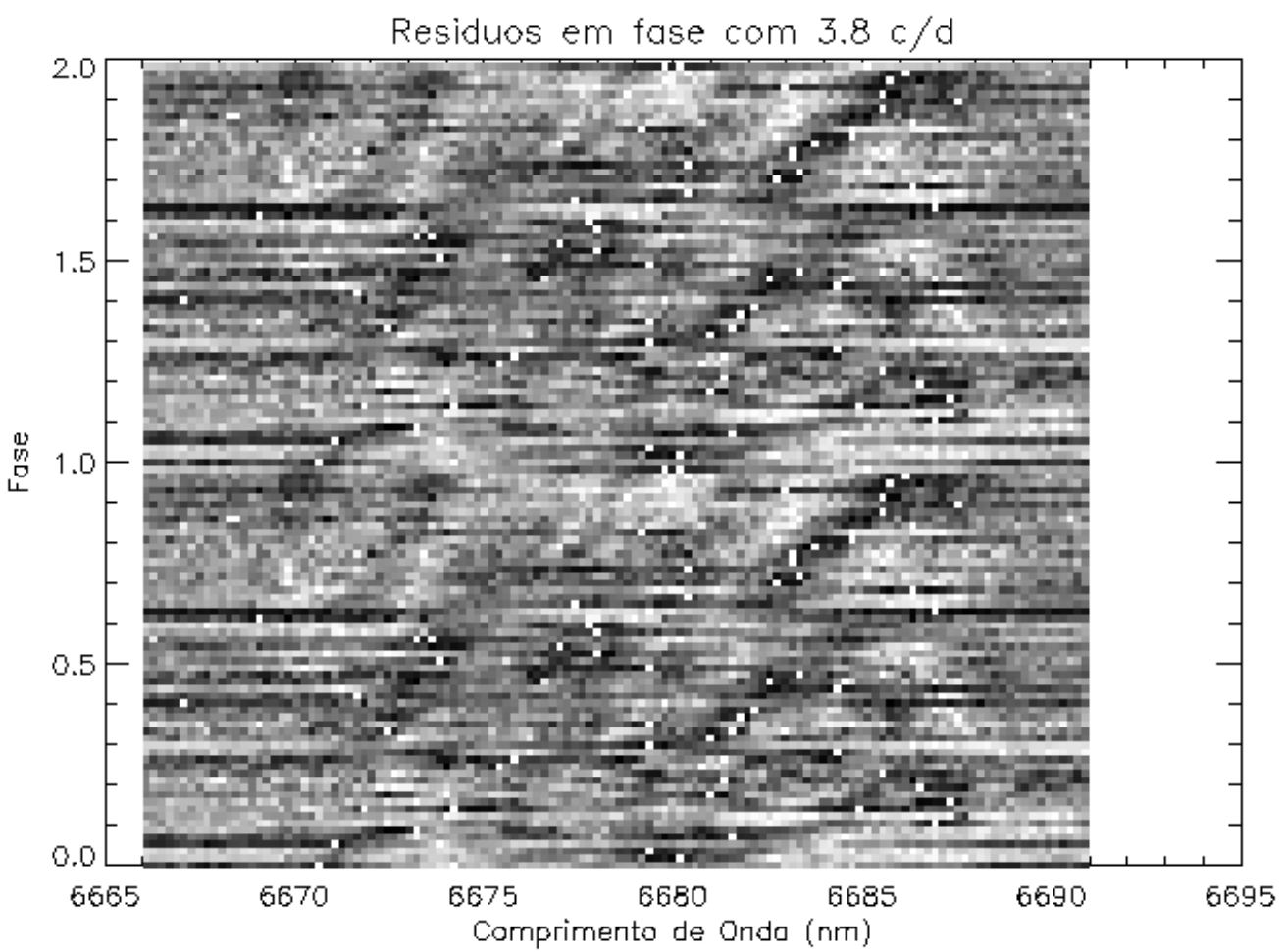

Fig.41. Diagrama em escala de cinza mostrando os resíduos médios dos espectros centrados em $\lambda 667.8 \mathrm{~nm}$, em fase com a freqüência de 3.8 ciclos/dia. 


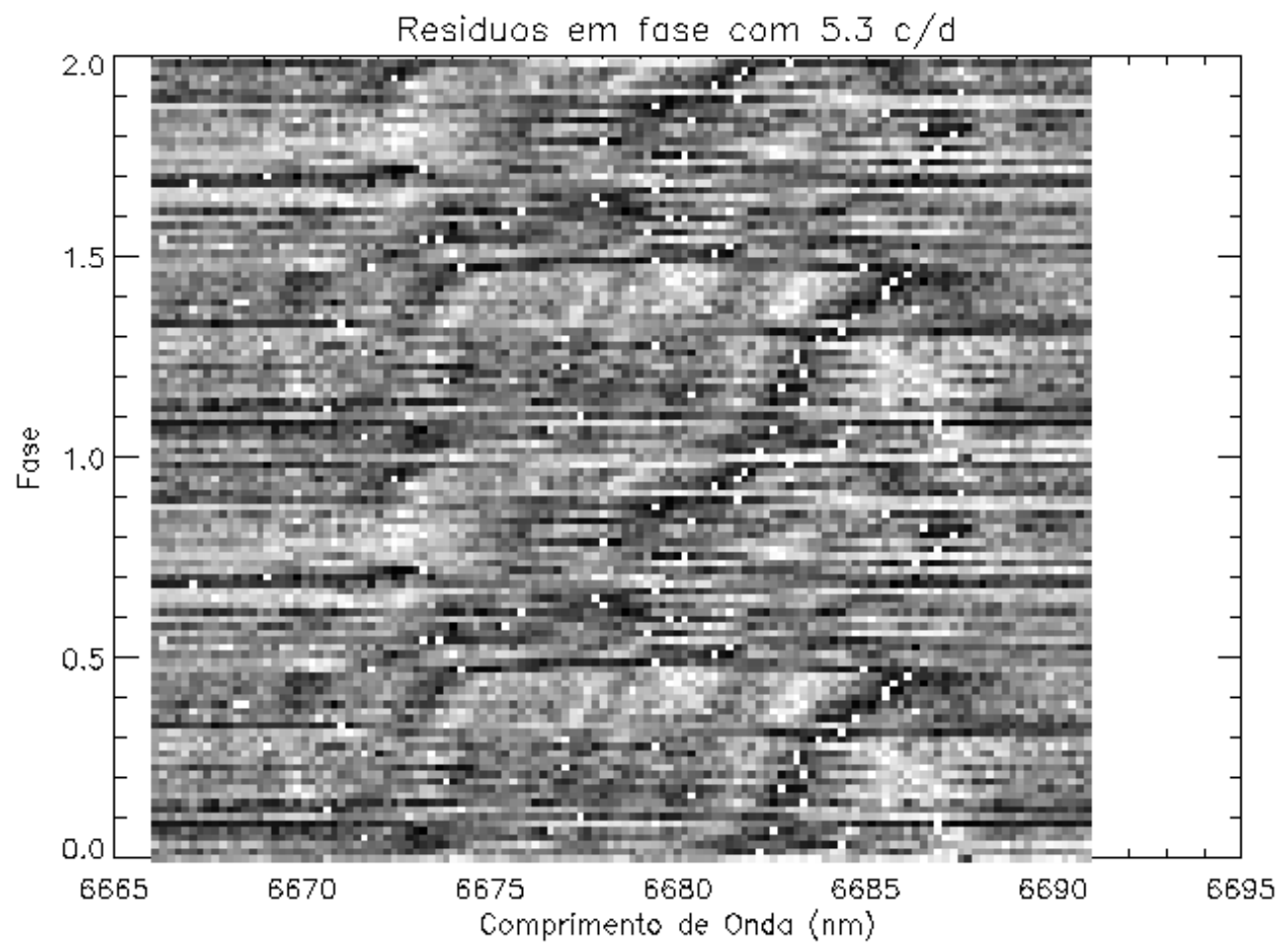

Fig.42. Diagrama em escala de cinza mostrando os resíduos médios dos espectros centrados em $\lambda 667.8 \mathrm{~nm}$, em fase com a freqüência de 5.3 ciclos/dia.

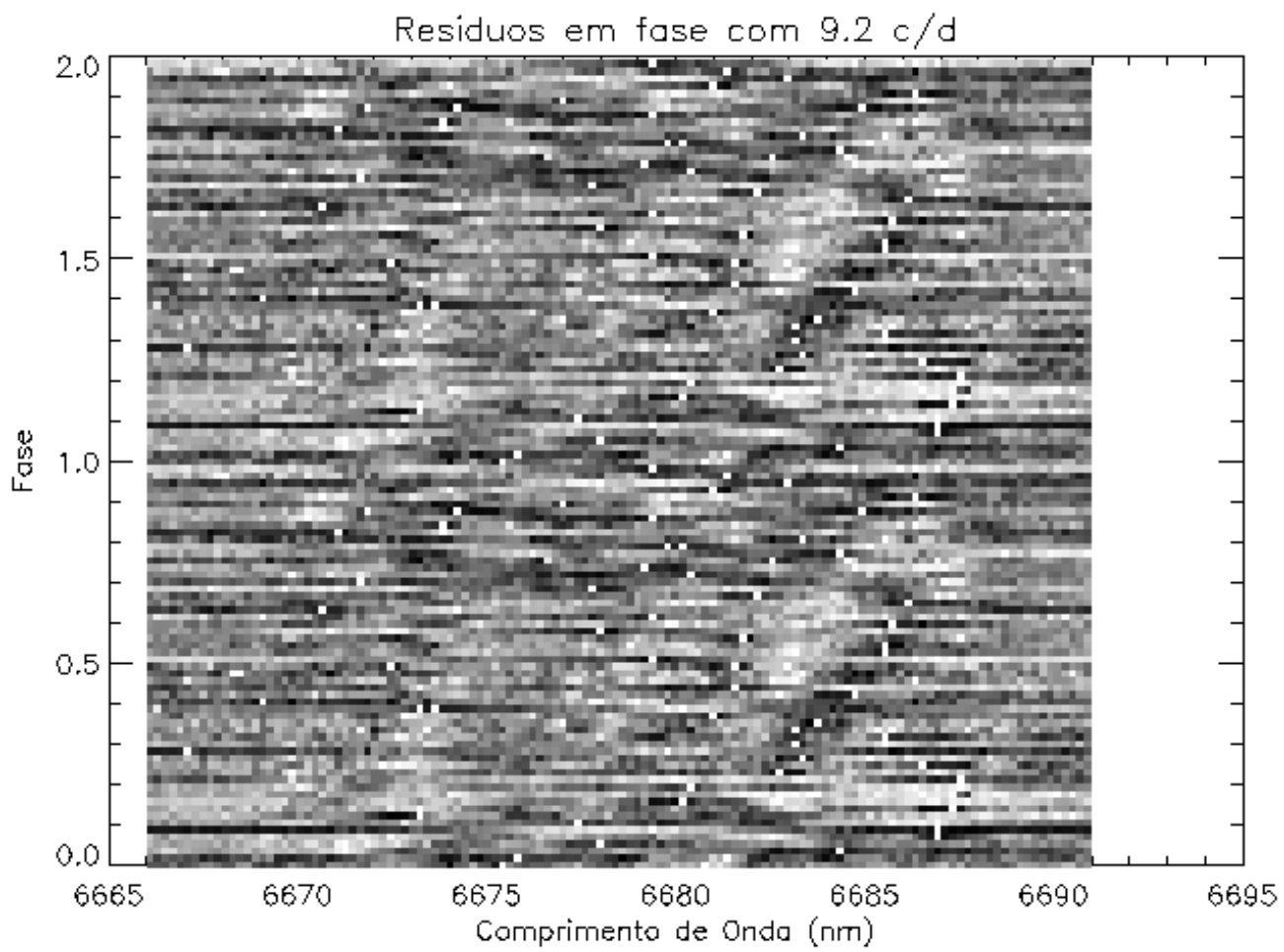

Fig.43. Diagrama em escala de cinza mostrando os resíduos médios dos espectros centrados em $\lambda 667.8 \mathrm{~nm}$, em fase com a freqüência de 9.2 ciclos/dia. 


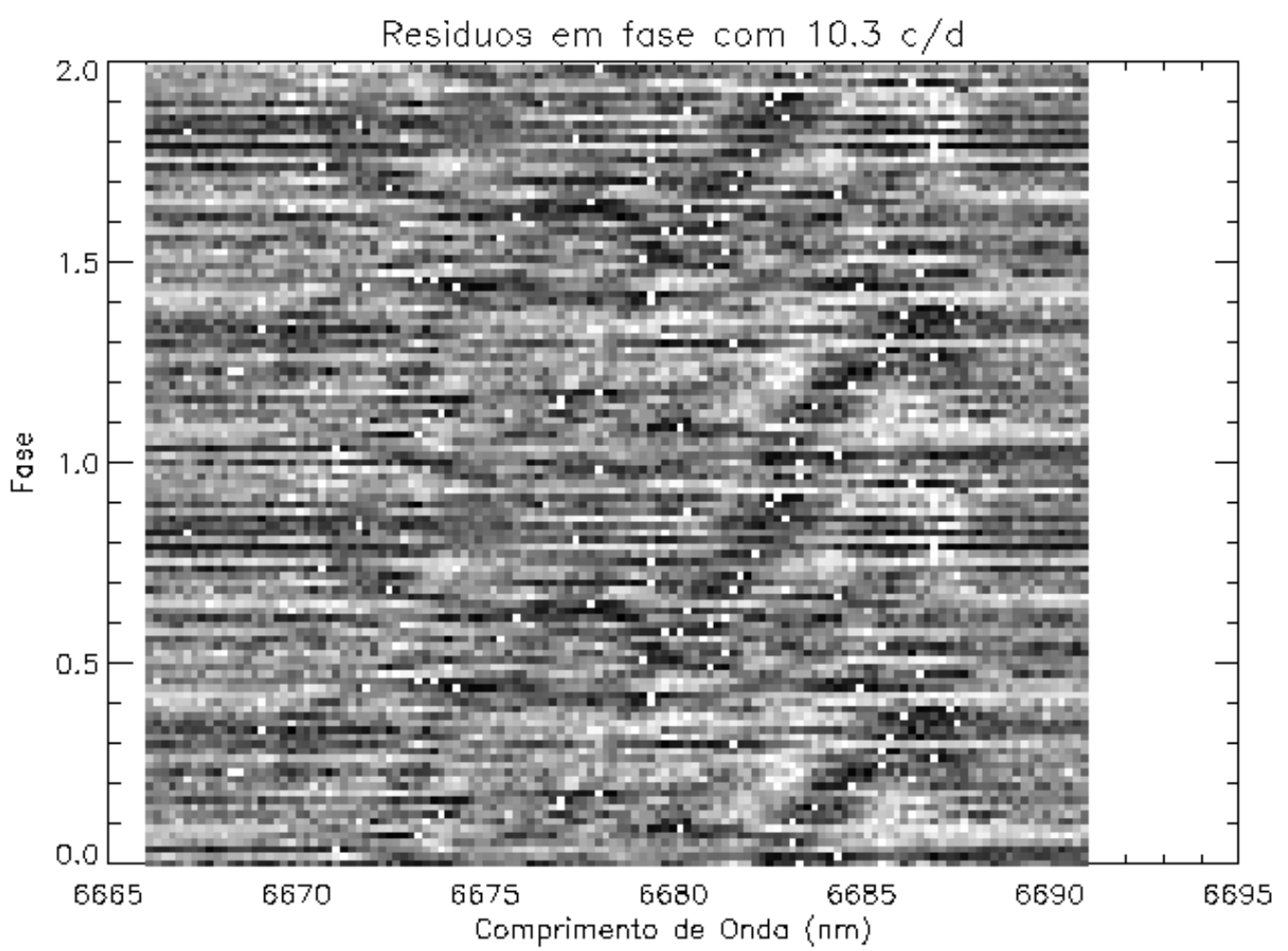

Fig.44. Diagrama em escala de cinza mostrando os resíduos médios dos espectros centrados em $\lambda 667.8 \mathrm{~nm}$, em fase com a freqüência de 10.3 ciclos/dia.

Nos diagramas anteriores, pode-se observar a passagem dos bumps ao longo do perfil de linha do azul para o vermelho. A forma exibida no diagrama correspondente às freqüências de $0.6 \mathrm{c} / \mathrm{d}$ e $1.48 \mathrm{c} / \mathrm{d}$ sugerem possivelmente oscilações de modo setorial. As freqüências mais altas têm um comportamento mais complicado, associado talvez a modos tesserais.

\subsection{Aplicação do Método de Telting \& Schrijvers}

Com o intuito de investigar as informações contidas nas $l p v$, Telting \& Schrijvers (1997a, 1997b e 1997c) geraram perfis de linha teóricos deformados por PNR, onde foram considerados o efeito do campo de velocidade e das variações de temperatura. Os efeitos da temperatura foram introduzidos através de mudanças na largura equivalente de um perfil intrínseco gaussiano. Além disso, eles consideraram também os efeitos de uma rotação estelar baixa sobre os modos de PNR. Foram gerados vários conjuntos de perfis de linha teóricos, variando-se os diversos parâmetros estelares e de pulsação (i, $\lambda, \mathrm{m}, \mathrm{k}$, q, $\omega, \mathrm{W}$, etc.) do modelo de PNR adotado. Cada conjunto de linhas foi obtido introduzindo-se apenas um modo de PNR. Em seguida, cada conjunto de espectros foi analisado de modo similar ao método de Gies \& Kullavanijaya (1988), sendo que neste caso a freqüência de oscilação já é conhecida. Além disso, foi levado em conta que o mapeamento Doppler da superfície tridimensional da estrela sobre um espaço 
unidimensional de velocidades causa lpv não apenas com a freqüência observada, mas também com seus harmônicos. Desse modo, para cada série temporal definida por um comprimento de onda fixo, foi ajustada uma função do tipo

$$
y(\lambda, t)=y_{0}(\lambda, t) \sin \left(\omega_{o b s} t-\phi_{0}(\lambda)\right)+y_{1}(\lambda, t) \sin \left(2 \omega_{o b s} t-\phi_{1}(\lambda)\right)
$$

onde y representa o resíduo da série temporal (intensidade menos a intensidade média), $\mathrm{y}_{0}, \phi_{0}$ são a amplitude e fase da freqüência fundamental, e $\mathrm{y}_{1}$ e $\phi_{1}$ são a amplitude e fase do primeiro harmônico da freqüência fundamental. Os harmônicos de ordem superior foram desprezados por apresentarem, em geral, amplitudes abaixo do nível de ruído em análises de espectros reais.

Normalmente, $\mathrm{y}_{0}>\mathrm{y}_{1}$, entretanto para alguns conjuntos particulares de parâmetros estelares e de pulsação, essa relação pode até ser invertida. A seguir, uma análise dos conjuntos de perfis de linha teóricos através do método de Monte Carlo levou à seguinte relação (desprezando-se as constantes):

$$
\begin{gathered}
\lambda \approx \frac{\left|\Delta \phi_{0}\right|}{\pi} \\
|m| \approx \frac{\left|\Delta \phi_{1}\right|}{2 \pi}
\end{gathered}
$$

onde $\left|\Delta \phi_{0}\right|$ e $\left|\Delta \phi_{1}\right|$ representam a máxima diferença de fase associada à freqüência fundamental e ao seu primeiro harmônico. As diferenças de fase devem ser medidas na região onde as $l p v$ são detectadas, podendo ultrapassar os limites de \pm vsini dependendo da fase de atividade da estrela (Kambe et al. 1993). As incertezas nos valores de $\lambda$ e $|m|$ calculados por esse método são \pm 1 e \pm 2 , respectivamente.

A determinação dos valores de $\lambda$ e $|\mathrm{m}|$ é possível para uma larga faixa de parâmetros, porém existem algumas limitações. Por exemplo, devemos ter $\lambda \leq 15$ e $|m| \leq$ 10 , bem como inclinações não muito próximas a $90^{\circ}$.

É interessante notar que a diferença de fase $\left|\Delta \phi_{0}\right|$ traz informações sobre $\lambda$ e não sobre $|\mathrm{m}|$, conforme sugerido pelos métodos dos resíduos e de Gies \& Kullavanijaya.

O método de Telting \& Schrijvers constitui, desse modo, uma ferramenta importante para estimar os parâmetros $\lambda$ e $|\mathrm{m}|$ associados a uma freqüência observada, sendo válido na presença de vários modos de pulsação, inclusive os tesserais. É importante notar porém que as relações (5.2) e (5.3) são resultado da análise de espectros sintéticos gerados com a teoria de pulsações para estrelas de rotação baixa e portanto a aplicação desse método para estrelas como $\eta$ Centauri deve ser realizada com cautela.

Como esse método utiliza as variações de fase associadas às freqüências dos modos de pulsação não radiais e de seus respectivos primeiros harmônicos, torna-se necessário primeiramente definir quais freqüências serão utilizadas.

De forma a estimar os parâmetros pulsacionais, são mostrados a seguir os diagramas de amplitude e fase para cada uma das freqüências determinadas anteriormente. 


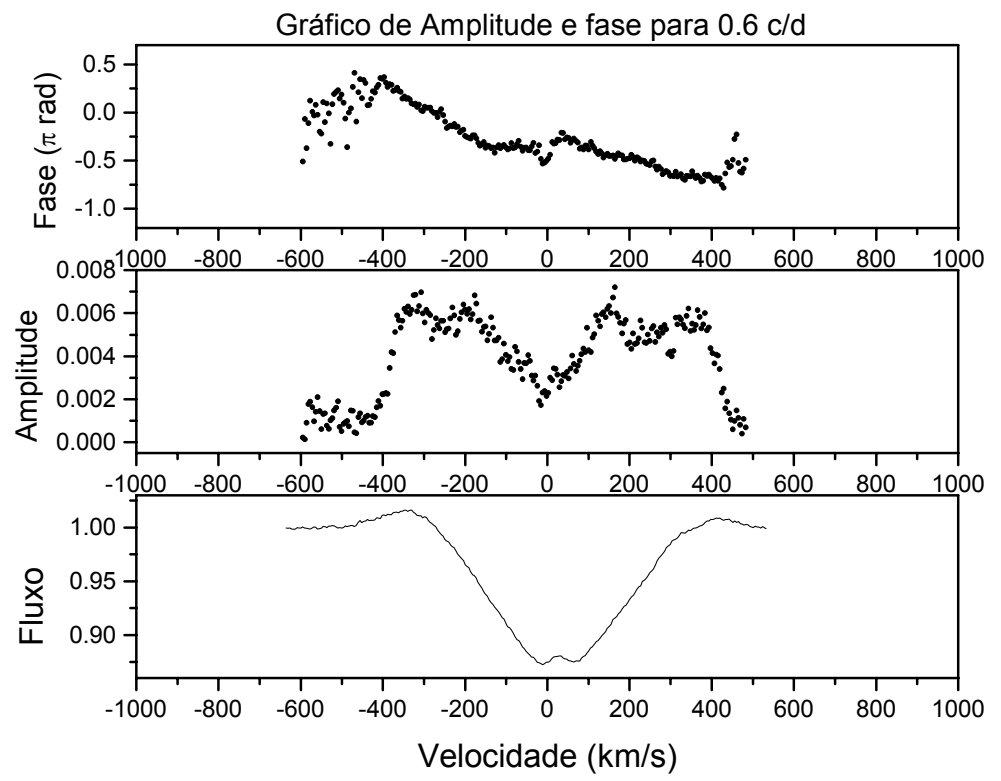

Fig.45. Amplitudes e fases da freqüência $0.6 \mathrm{c} / \mathrm{d}$ ao longo do perfil de linha do HeI. O aspecto do diagrama de amplitude sugere ser esta freqüência associada a uma oscilação de modo g. A partir do diagrama de fase, podemos estimar $\Delta \phi \sim-\pi$ e o grau da pulsação $\lambda \sim 1$ (Telting et al., 1997).

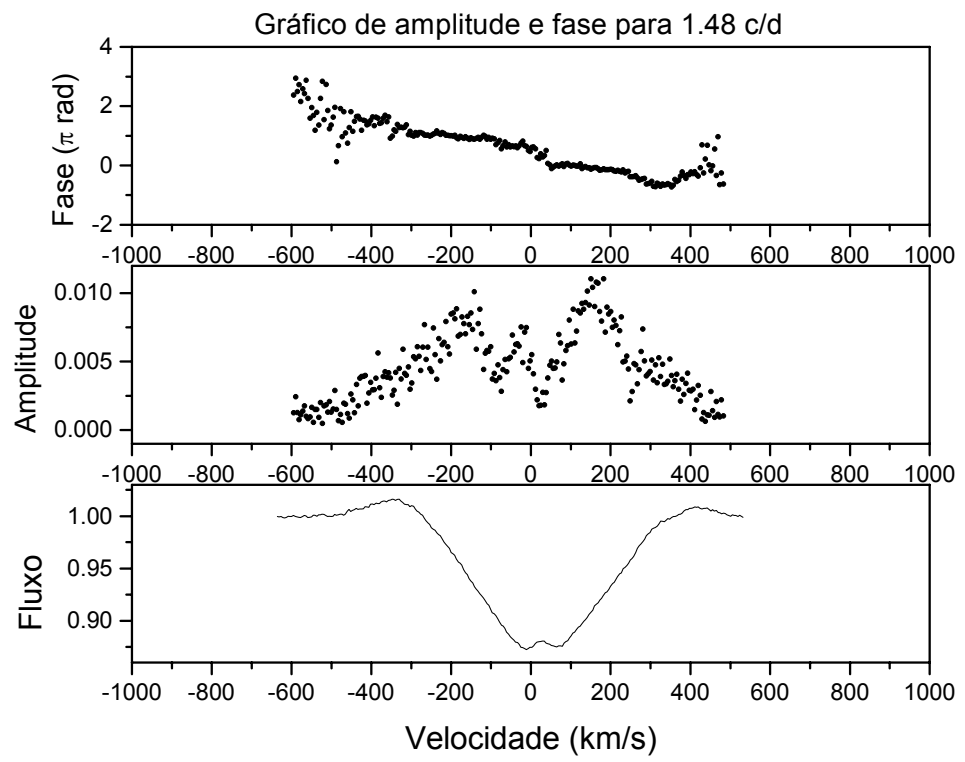

Fig.46. Amplitudes e fases da freqüência $1.48 \mathrm{c} / \mathrm{d}$ ao longo do perfil de linha do HeI. O aspecto do diagrama de amplitude sugere ser esta freqüência associada a uma oscilação de modo g. A partir do diagrama de fase, podemos estimar $\Delta \phi \sim--3 \pi$ e o grau da pulsação como sendo $\lambda \sim 3$ (Telting et al., 1997). 


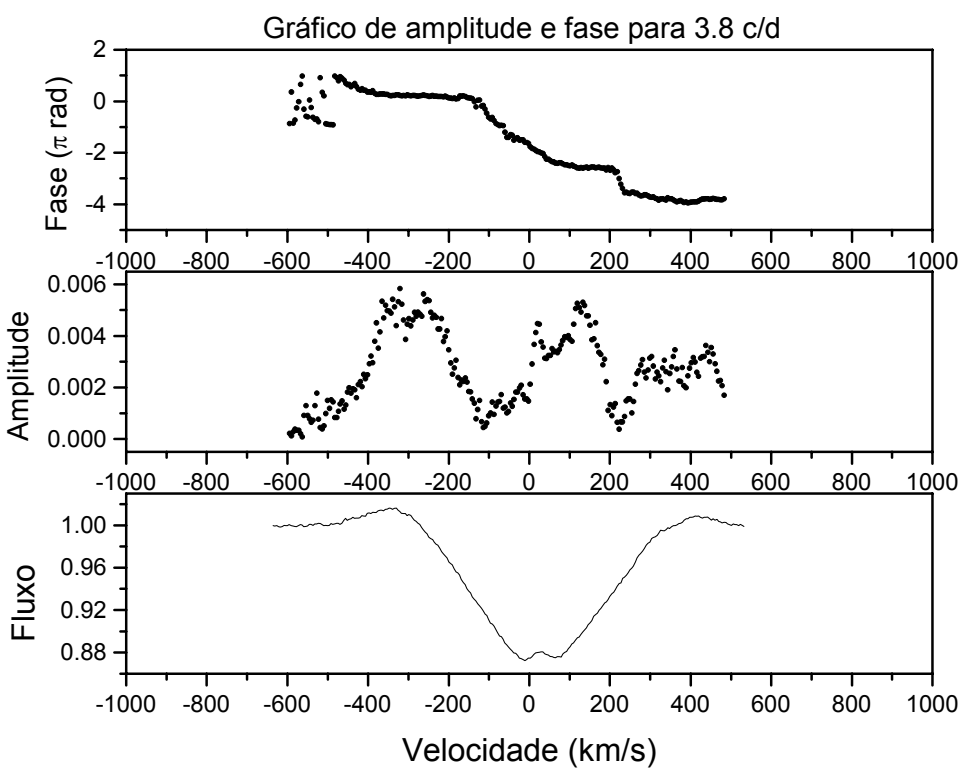

Fig.47. Amplitudes e fases da freqüência $3.8 \mathrm{c} / \mathrm{d}$ ao longo do perfil de linha do HeI. A partir do diagrama de fase, podemos estimar $\Delta \phi \sim-5 \pi$ e o grau da pulsação $\lambda \sim 5$ (Telting et al, 1997).

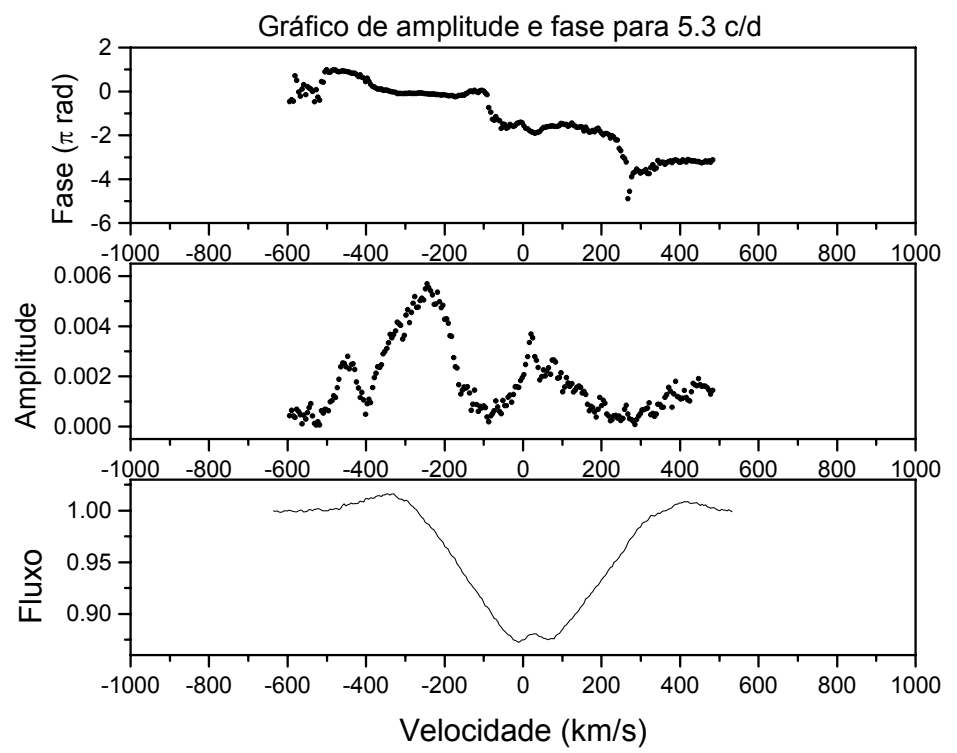

Fig.48. Amplitudes e fases da freqüência $5.3 \mathrm{c} / \mathrm{d}$ ao longo do perfil de linha do HeI. A partir dos diagramas de fase, podemos estimar $\Delta \phi \sim-5 \pi$ e o grau da pulsação $\lambda \sim 5$ (Telting et al., 1997). 


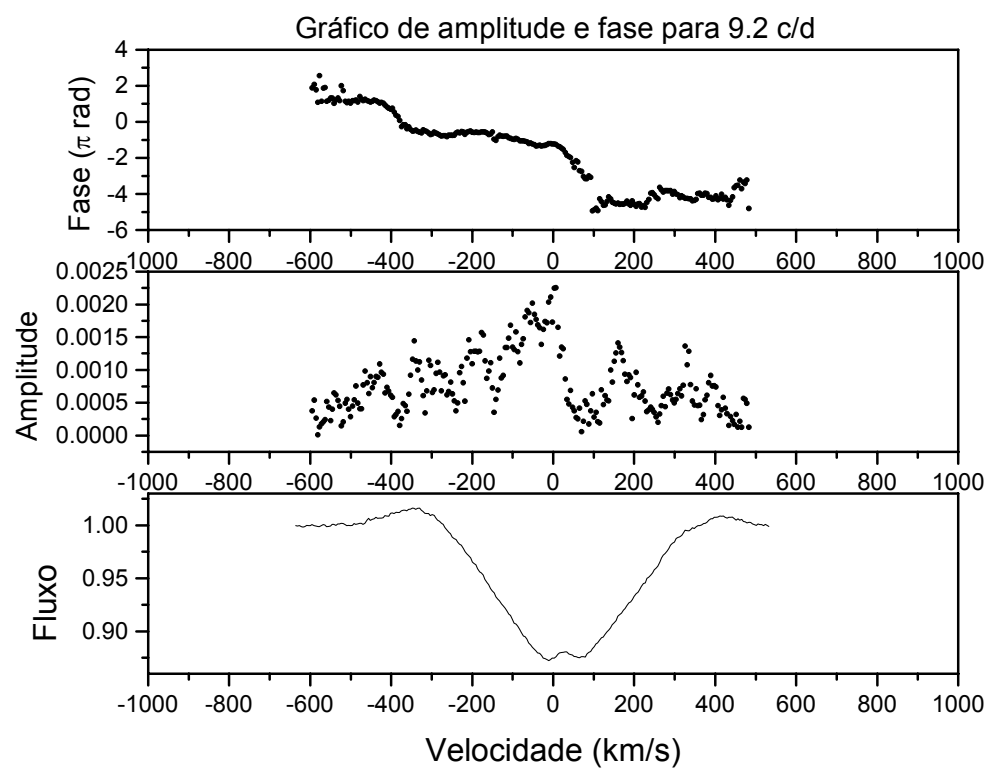

Fig.49. Amplitudes e fases da freqüência $9.2 \mathrm{c} / \mathrm{d}$ ao longo do perfil de linha do HeI. A partir do diagrama de fase, podemos estimar $\Delta \phi \sim-5 \pi$ e o grau da pulsação $\lambda \sim 5$ (Telting et al., 1997).

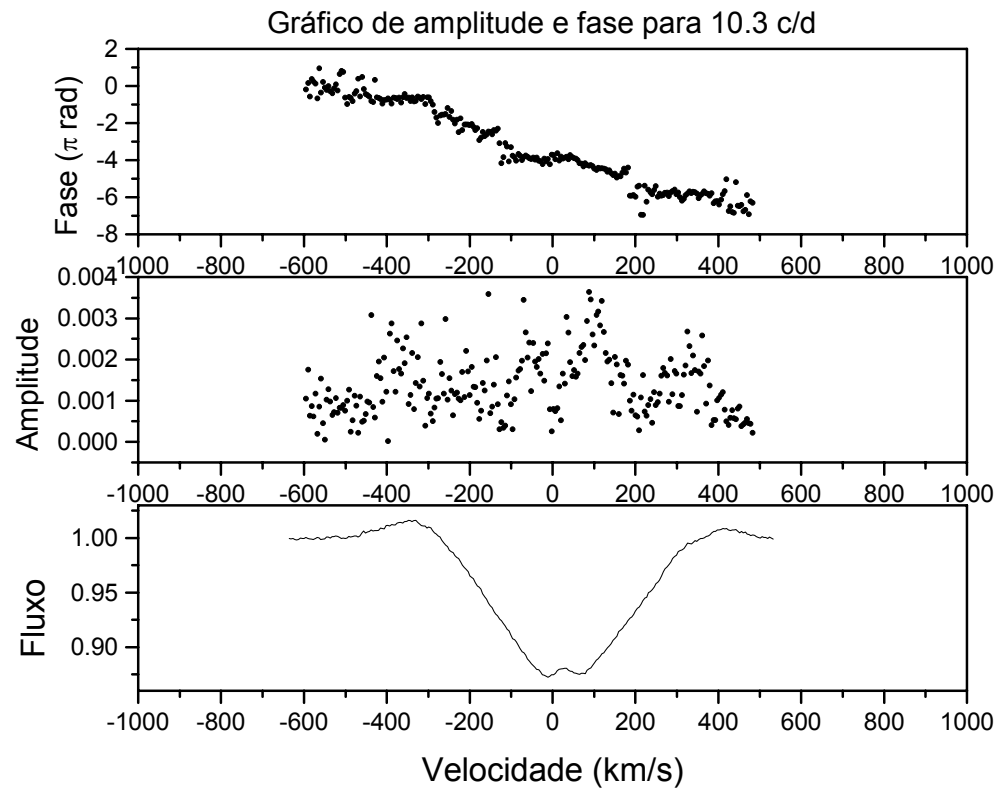

Fig.50. Amplitudes e fases da freqüência $10.3 \mathrm{c} / \mathrm{d}$ ao longo do perfil de linha do HeI. A partir do diagrama de fase, podemos estimar $\Delta \phi \sim-7 \pi$ o grau da pulsação como sendo $\lambda \sim 7$ (Telting et al., 1997). 
A partir das análises dos diagramas de amplitude e fase, podemos estimar os parâmetros pulsacionais da estrela, e resumi-los na Tabela 23. Note que, além dos erros associados a $\lambda( \pm 1)$, provenientes do próprio desenvolvimento do método, há também uma incerteza associada à determinação das diferenças de fase, uma vez que nem sempre é simples definir o limite até onde as fases variam de maneira coerente. Entretanto, na maioria das vezes, as incertezas do método devem ser maiores do que a incerteza na medida das variações de fase.

\begin{tabular}{ccc}
\hline $\begin{array}{c}\text { Freqüência } \\
(\mathrm{c} / \mathrm{d})\end{array}$ & $\Delta \phi(\mathrm{rad})$ & $\lambda( \pm 1)$ \\
\hline 0.6 & $-\pi$ & $\sim 1$ \\
1.48 & $-3 \pi$ & $\sim 3$ \\
3.8 & $-5 \pi$ & $\sim 5$ \\
5.3 & $-5 \pi$ & $\sim 5$ \\
9.2 & $-5 \pi$ & $\sim 5$ \\
\hline
\end{tabular}

Tab. 23. Resumo dos parâmetros pulsacionais obtidos a partir da análise dos diagramas de amplitude e fase.

As freqüências $0.6 \mathrm{c} / \mathrm{d}$ e $1.48 \mathrm{c} / \mathrm{d}$ são devidas a oscilações de modo g, o que fica evidenciado a partir da forma dos diagramas de amplitude. Como $0.6 \mathrm{c} / \mathrm{d}$ é uma freqüência baixa, existe a possibilidade de ela estar relacionada com a atividade do envelope. A freqüência $3.8 \mathrm{c} / \mathrm{d}$ também pode ser um modo g, não obstante faltar o complemento em amplitude do lado vermelho, o qual pode estar mascarado pela atividade do envelope circunstelar (Figura 47). As freqüências de $5.3 \mathrm{c} / \mathrm{d}$ e $10.3 \mathrm{c} / \mathrm{d}$ são efetivamente tesserais, onde $10.3 \mathrm{c} / \mathrm{d}$ é o primeiro harmônico de $5.3 \mathrm{c} / \mathrm{d}\left(v_{2} \sim 2 v_{1}\right)$ com $\Delta \phi \sim-7 \pi$. Nesse caso, aplicando-se a relação (5.3), temos um modo g com $\lambda \sim 5 \pm 1$ e $|m|$ $\sim 3 \pm 2$ (modo tesseral). A freqüência $9.2 \mathrm{c} / \mathrm{d}$ pode ser atribuída a uma oscilação de modo p, embora exista uma depressão no gráfico de amplitude ocasionada pela diminuição do sinal nessa região (observada como uma descontinuidade da fase do sinal na Figura 49). 


\subsection{Análise de Dados de Fotometria}

Embora o objetivo deste trabalho seja o estudo de variabilidades espectroscópicas, a análise de dados de fotometria pode trazer importantes subsídios para a obtenção do período de rotação da estrela (Balona, 1990; Balona, 1995).

Os dados fotométricos analisados neste trabalho foram obtidos pelo satélite HIPPARCOS ao longo dos anos de 1990, 1991 e 1992, e são mostrados na figura 51.

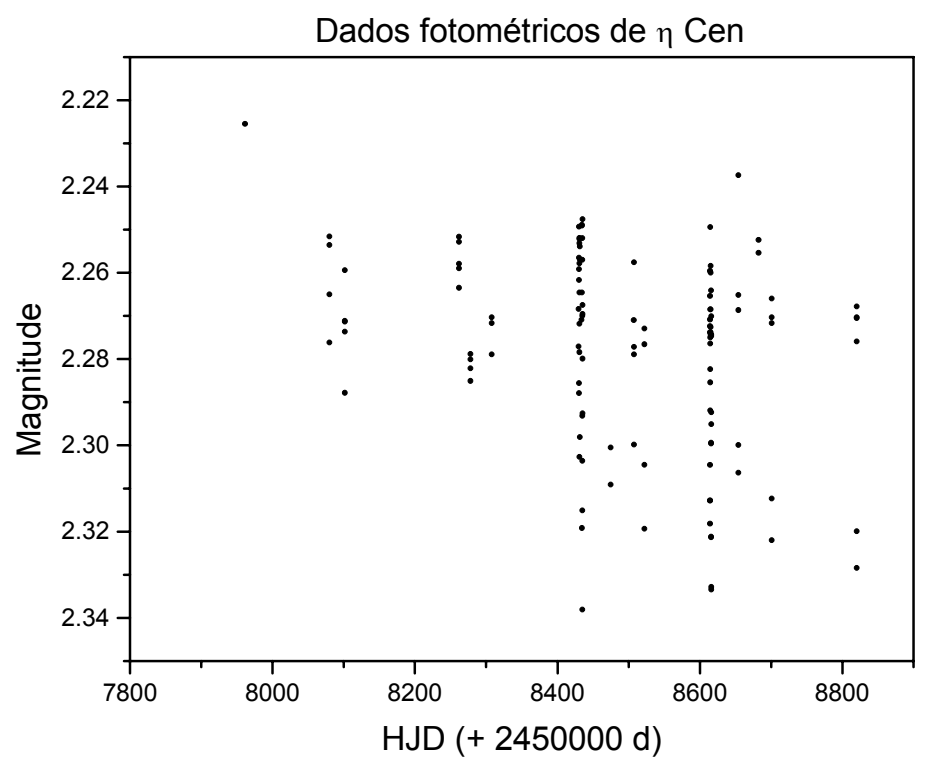

Fig.51. Curva de luz dos dados fotométricos obtidos pelo satélite HIPPARCOS ao longo dos anos de 1990, 1991 e 1992.

Usando-se o método da transformada de Fourier e aplicando-se o critério CLEANEST de seleção de freqüências, obtemos para essa estrela uma freqüência de $1.55 \mathrm{c} / \mathrm{d}$ (mais significativa entre 3 freqüências, $\chi^{2}=16.2$, significado $=97.5 \%$ ), próxima ao valor de $1.48 \mathrm{c} / \mathrm{d}$ obtido pela espectroscopia. Essa freqüência detectada na fotometria pode estar relacionada com a freqüência de rotação da estrela (Balona, 1995; Balona et al., 1999). 


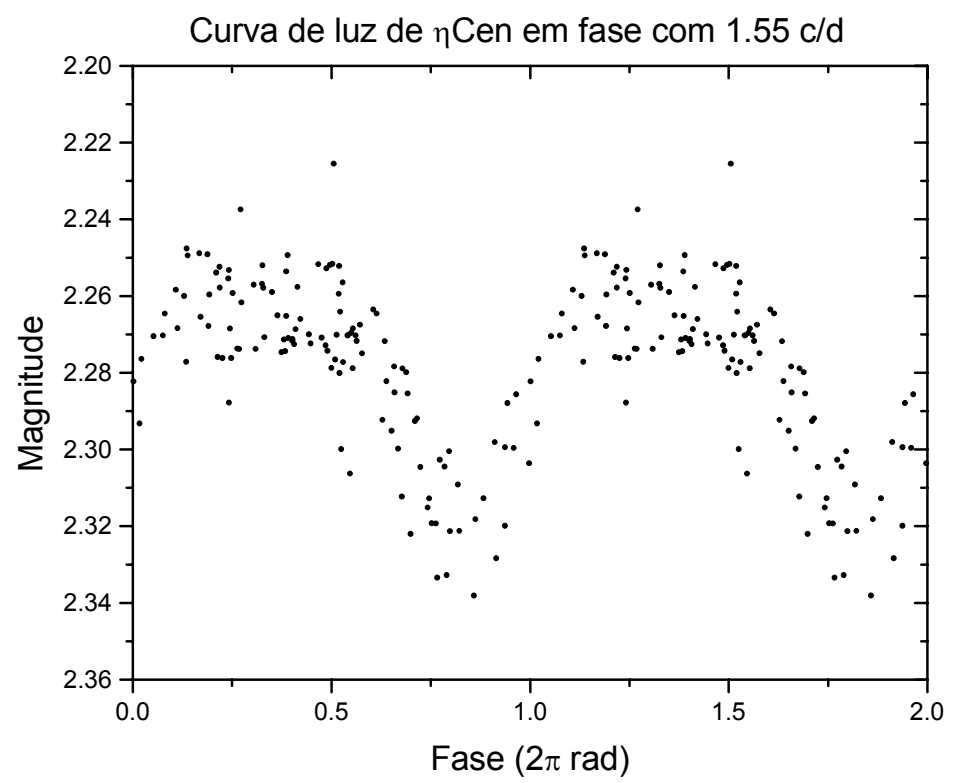

Fig.52. Curva de luz dos dados fotométricos obtidos pelo satélite HIPPARCOS, em fase com a freqüência de $1.55 \mathrm{c} / \mathrm{d}$, exibindo dois ciclos completos de oscilação $(0$ a $4 \pi \mathrm{rad})$.

A estimativa da freqüência crítica da estrela (break-up) depende de sua massa e de seu raio. Valores extremos desses parâmetros para estrelas B são dados por Harmanec (1988) e Underhill (1982). Adotando-se as dimensões físicas de uma estrela B1-2V, segundo Harmanec, temos: $\mathrm{R}=4.3-4.9 \mathrm{R}_{\odot}$, $\mathrm{M}=8.6-11.0 \mathrm{M}_{\odot} . \mathrm{Na}$ aproximação de Roche, uma estrela que gira com velocidade crítica deve ter raio $R_{c}=1.5 R *$ Com os parâmetros de massa e raio acima, as freqüências de rotação crítica serão $v_{\text {crit }}=2.6 \mathrm{c} / \mathrm{d}$ $\left(\mathrm{V}_{\text {crit }}=570 \mathrm{~km} \mathrm{~s}^{-1}\right)$ (usando-se a maior massa e o menor raio) e $v_{\text {crit }}=1.89 \mathrm{c} / \mathrm{d}\left(\mathrm{V}_{\text {crit }}=472\right.$ $\mathrm{km} \mathrm{s}^{-1}$ ) (usando-se a menor massa e o maior raio). Baseado em observações no UV, Hutchings et al. (1979) estimaram as velocidades de rotação e os ângulos de inclinação de uma amostra de estrelas O-B. Para $\eta$ Cen, eles obtiveram $i=55^{\circ}\left(+12^{\circ},-7^{\circ}\right)$ e $V_{\text {rot }} / V_{\text {crit }}$ $=0.65(+0.07,-0.12)$. Considerando esses valores verdadeiros, e assumindo vsini $=350$ $\mathrm{km} \mathrm{s}^{-1}$, temos, para $\mathrm{R}_{\mathrm{c}}=\mathrm{R}_{*}$, uma velocidade equatorial $\mathrm{V}_{\mathrm{e}}=427 \mathrm{~km} \mathrm{~s}^{-1}$. A freqüência crítica de $\eta$ Cen é, provavelmente, $v_{\text {crit }} \leq 2.2 \mathrm{c} / \mathrm{d}\left(\mathrm{P}_{\text {crit }} \geq 0.45 \mathrm{c} / \mathrm{d}\right)$, que corresponde à média dos valores de freqüência crítica calculados acima e está de acordo com o obtido por Janot et al (1999). A definição desses parâmetros é importante na interpretação das periodicidades encontradas nas análises das séries temporais. Por exemplo, freqüências maiores do que $\sim 2.2 \mathrm{c} / \mathrm{d}$ não são devidas unicamente à rotação estelar. Assim, assumindo-se que o período fotométrico esteja relacionado com a rotação da estrela, ele é cerca de $70 \%$ menor que freqüência crítica e os efeitos da rotação são insuficientes para assegurar a perda de matéria. 


\subsection{Análise da Variabilidade Cíclica em V/R}

Uma característica peculiar das estrelas Be é a presença de um envelope circunstelar "frio" que se torna aparente nos comprimentos de onda da região do óptico (hidrogênio, hélio e, às vezes, linhas de emissão metálicas) e do infravermelho (emissões contínuas bound-free e emissões térmicas free-free). Nas estrelas com altas velocidades de rotação, existem fortes evidências de que a região emissora possui formato de disco concentrada na região do equador estelar. Cerca de $20 \%$ das Be's mostram linhas finas de absorção metálicas não-fotosféricas (linhas shell) além das linhas de emissão, ou pelo menos linhas de hidrogênio com depressões centrais, que indicam que a luz proveniente da fotosfera foi absorvida pelo envelope ao longo da linha de visão. Nas $80 \%$ restantes a linha de visão não intercepta o envelope, possuindo em média ângulos de abertura da ordem de $\mathrm{i} \sim 12^{\circ}$ (Hanuschik, 1994). Essa geometria em forma de disco foi confirmada independentemente através de observações interferométricas (Mourard et al. 1989; Quirrenbach 1994; Vakili et al. 1994). Entretanto, as condições físicas nesses discos, como seus estados cinemáticos e distribuições de densidade, continuam em aberto (Hanuschik, 1995).

A relação $\mathrm{V} / \mathrm{R}$ apresenta variações a todo tempo, sendo talvez mais pronunciada em momentos próximos à ocorrência de um outburst (Rivinius, 1998) e é melhor observada em linhas de He I tais como $\lambda \lambda 587.6$, 667.8 e $706.5 \mathrm{~nm}$. É importante notar que a emissão de linhas de Fe II (como $\lambda \lambda 516.9$ e $531.7 \mathrm{~nm}$ ) se torna detectável somente no pico de um outburst, aparecendo de forma súbita e persistindo por toda a fase de outburst. A distinção entre um outburst maior e um menor é bem clara a partir da análise das linhas de Fe II: enquanto um burst maior leva a uma emissão persistente, em um burst menor a emissão cessa quando termina o burst.

Neste trabalho, foram estudadas as variações em V/R apenas da linha $\lambda 667.8 \mathrm{~nm}$, ao longo de 4 anos de observação (1995, 1996, 1997 e 1998). Foi constatada uma intensa atividade nas asas dessa linha, com variações da ordem de $3 \%$ em relação à intensidade do contínuo. A partir dos espectros de $\mathrm{H} \alpha$ relativos a esse período, pode-se inferir que a estrela se encontra em fase shell, entretanto não se pode precisar o início e a duração do período de outburst a partir desses espectros. Devido à curta amostragem (4 anos), também não se pode inferir nada sobre as modulações de longo período das variabilidades V/R (da ordem de décadas). Dessa forma, foram determinadas apenas as variabilidades rápidas, com períodos de horas-dias.

A presença de multiperiodicidades nas análises das séries temporais de $\mathrm{V} / \mathrm{R}$ pode representar um indício da existência de um envelope circunstelar com distribuição inomogênea de densidades, ou ainda a presença de várias nuvens em órbita na estrela, a diferentes distâncias da mesma. Vamos assumir neste trabalho a existência, em $\eta$ Cen, de um único disco circunstelar com distribuição não homogênea de densidades, conforme ilustra a Figura 53. 


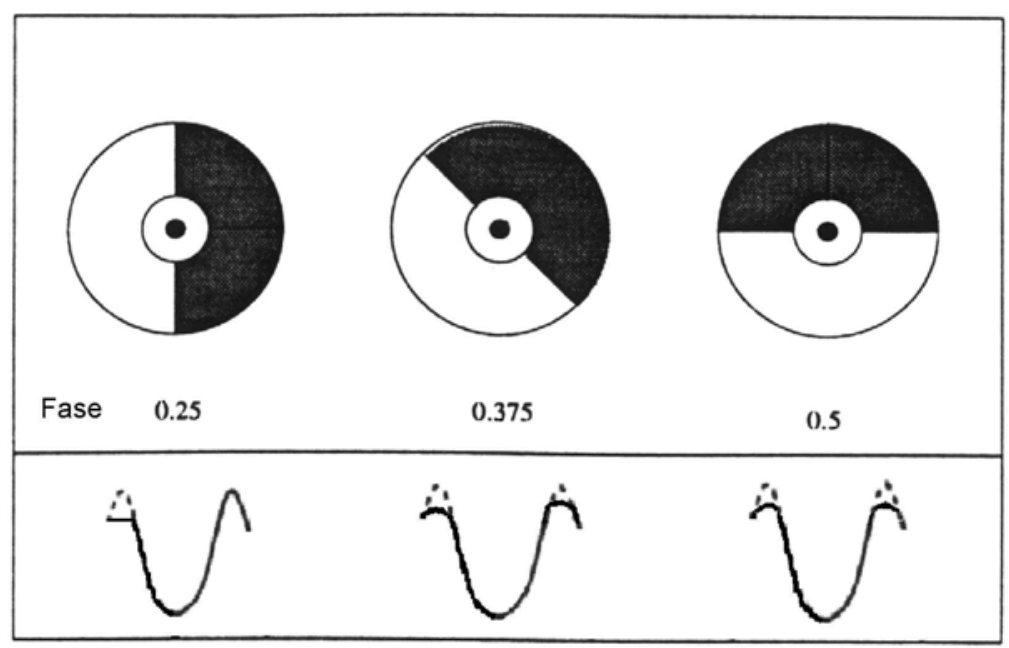

Fig.53. Esquema ilustrativo do mecanismo de variabilidades rápidas nas asas da linha do HeI. A região escura em torno da estrela representa parte do disco circunstelar com maior densidade de matéria. No instante com fase 0.25 , a asa vermelha exibe o seu máximo de intensidade devido à passagem da nuvem pelo lado direito da estrela. À medida em que a nuvem se desloca em órbita pela estrela, a intensidade da asa vermelha diminui e a parte do disco que se aproxima contribui para o aumento de intensidade da asa violeta.

(adaptado de Štefl et al., 2000)

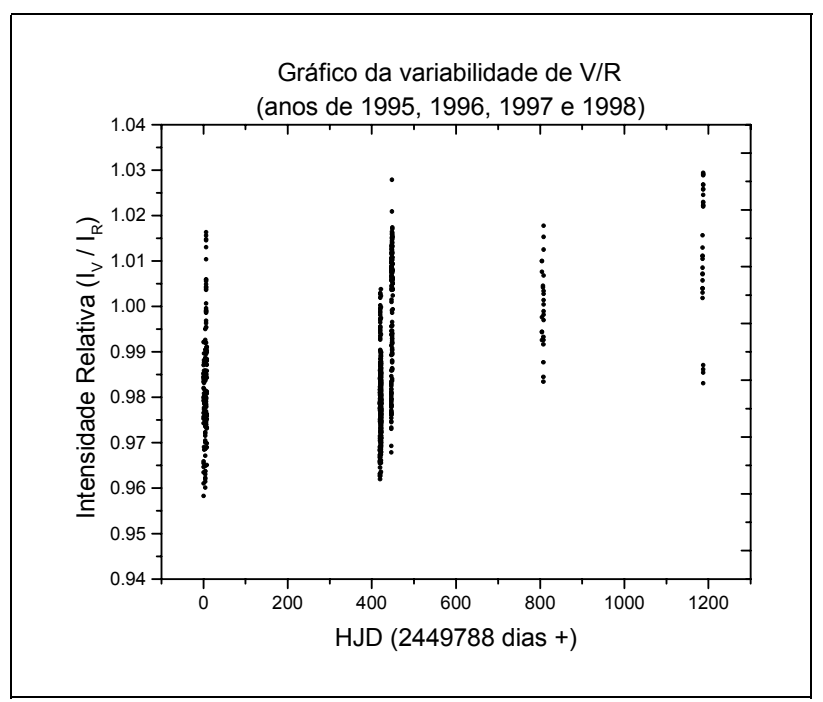

Fig.54. Gráfico da razão V/R relativa aos anos de 1995, 1996, 1997 e 1998

Pelo gráfico, quando a razão $\mathrm{V} / \mathrm{R}>1$, então a asa violeta da linha apresenta intensidade maior do que a asa vermelha, e vice-versa. Se $V / R=1$ então as intensidades de ambas são iguais. A amplitude pico a pico é da ordem de 3 a $4 \%$ em torno da unidade, valor semelhante ao encontrado na estrela Be $\mu$ Cen (Rivinius, 1998). 
Fazendo a seguir uma análise temporal para cada conjunto de dados em separado (ano a ano), obtemos os seguintes resultados:

\begin{tabular}{ccccc}
\hline Conjunto & $\begin{array}{c}\text { Freqüência }(\mathrm{c} / \mathrm{d}) \\
( \pm 0.01)\end{array}$ & $\begin{array}{c}\text { Graus de } \\
\text { liberdade }\end{array}$ & $\chi^{2}$ & Significado \\
\hline Todos os anos & 0.05 & 6 & 93 & $99.5 \%$ \\
1995 & 2.24 & 6 & 18.9 & $99.5 \%$ \\
1996 & 0.55 & 6 & 76.5 & $99.5 \%$ \\
$1997 / 1998$ & 1.56 & 6 & 8.5 & $75 \%$ \\
\hline
\end{tabular}

Tabela 24. Resultados obtidos aplicando o algoritmo CLEANEST às séries temporais de $\mathrm{V} / \mathrm{R}$ relativas aos anos de 1995, 1996, 1997 e 1998

As análises foram feitas buscando 3 períodos por vez, sendo que os apresentados na tabela são os mais significativos. Como a amplitude do espectro de potências do método CLEANEST é uma distribuição de $\chi^{2}$ de 2 graus de liberdade para cada período, então temos um número de graus de liberdade de 3 × $2=6$.

Devido à pequena amostragem temporal, o período mais longo detectado foi o de $\sim 20$ dias (0.05 c/d) referente ao conjunto de espectros de 1995/1996/1997/1998. Para os conjuntos individuais, a freqüência associada à variação de $\mathrm{V} / \mathrm{R}$ está compreendida no intervalo de $0.55 \mathrm{c} / \mathrm{d}-2 \mathrm{c} / \mathrm{d}$, comparável com o período de 0.62 dias $(1.6 \mathrm{c} / \mathrm{d})$ obtido para a estrela $\mu$ Cen (Rivinius, 1998). Esse intervalo de freqüências engloba a freqüência de rotação da estrela $(1.56 \mathrm{c} / \mathrm{d})$, constituindo uma variação de curto período possivelmente associada à passagem de matéria em órbita (blobs), pela linha de visão. 


\subsection{Análise da Variabilidade dos $C Q E$ 's}

Nos últimos anos, as observações de certas estrelas $\mathbf{B}$ e Be revelaram a presença de um tipo especial de perfil de linha, no qual a região central exibe um máximo local em fluxo. Esses acidentes no interior da linha são atualmente conhecidos como Central Quasi Emission Peaks (CQE's) e foram relatados primeiramente por Baade (1990) e Jeffery (1991) nas linhas do He I (B Early-Type) e do Mg II (B Late-Type), tendo sua origem sido considerada como sendo de natureza fotosférica. Existem atualmente vários modelos que procuram explicar sua origem, podendo-se dividi-los entre modelos fotosféricos e modelos circunstelares. Entre os fotosféricos, podemos citar o de Baade (1990) e Jeffery (1991), onde as variações, do equador até o polo estelar, da gravidade local e da temperatura na presença de rotação rápida seriam as responsáveis pela emissão central, e o modelo de rotação diferencial (Zorec, 1994), que supõe a temperatura efetiva e a gravidade constantes e uniformes ao longo da superfície estelar, negligenciando eventuais deformações geométricas devidas à rotação. Nesse contexto, os CQE's surgiriam devido à variação do comprimento das linhas de iso-velocidade radial do centro até as asas da linha (Figura 55).

No modelo de origem circunstelar, Hanuschik, (1995a) calculou os contornos de iso-velocidade radial em um disco gasoso visto edge-on e encobrindo uma fração da estrela. Neste caso, a formação dos $C Q E$ 's é devida a um mínimo de absorção em velocidade radial zero, correspondendo a um máximo em fluxo nessa região. 

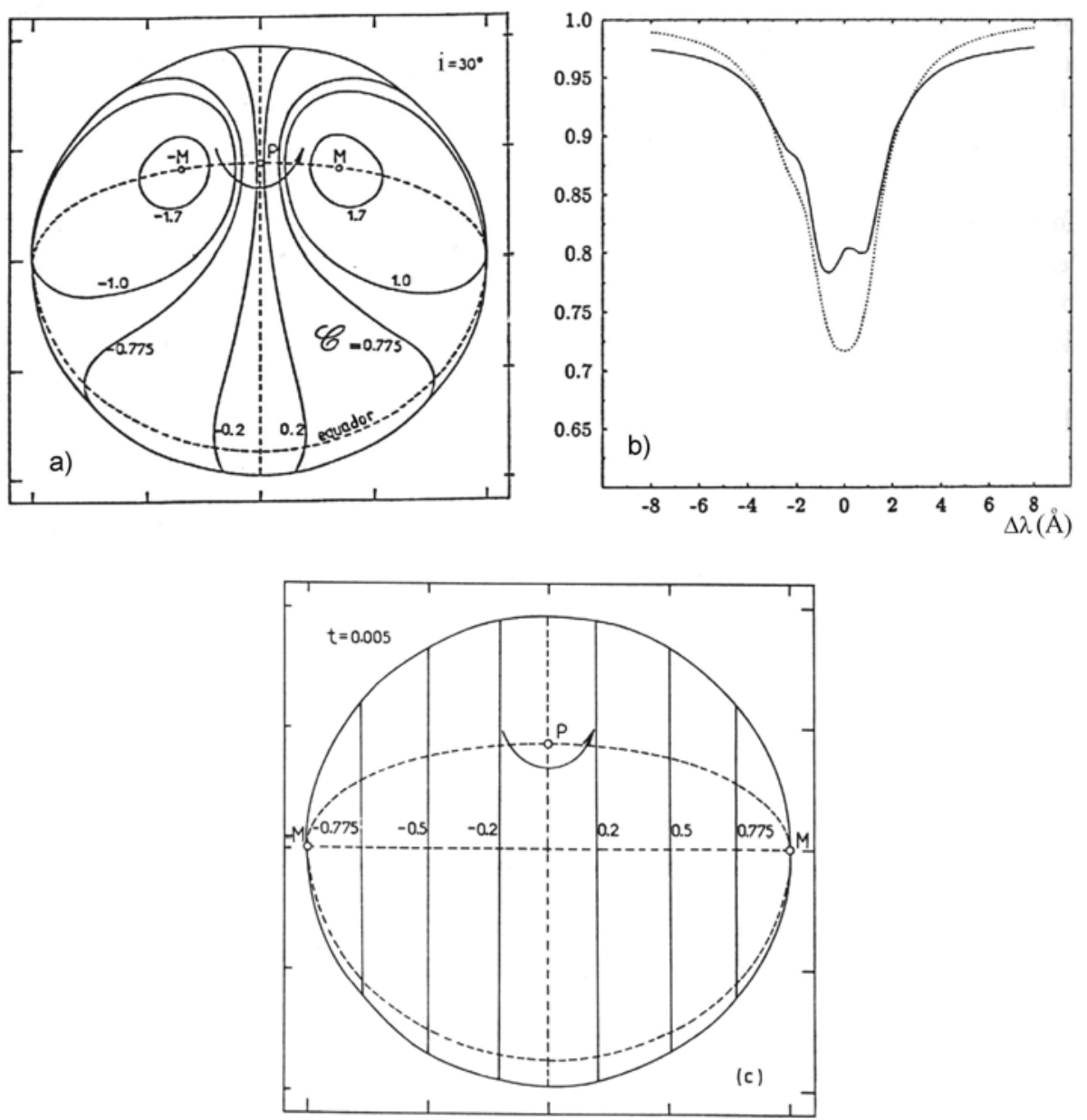

Fig.55.Modelo fotosférico (Zorec, 1994) a) (linhas cheias) Curvas de iso-velocidade radial para uma estrela vista com $\mathrm{i}=30^{\circ}$, onde $\mathrm{P}$ é o pólo estelar e.$\pm \mathrm{M}$ são os pontos de velocidade radial de valores $\pm V_{\mathrm{MAX}} \sin$ i. b) (linha cheia) Perfil da linha em Hel $\lambda 447.1 \mathrm{~nm}$ com $\mathrm{V}_{\mathrm{MAX}} \sin \mathrm{i}=100 \mathrm{~km} \cdot \mathrm{s}^{-1}$. (linha pontilhada) perfil alargado por rotação rígida e mesmo valor de $\mathrm{V} \sin$ i. c) Curvas de iso-velocidade radial com $\mathrm{i}=30^{\circ}$, para o caso de rotação rígida.

Os $C Q E$ 's estiveram presentes nos espectros analisados neste trabalho durante todo o período que vai de 1996 a 1998, que corresponde a um período shell. Foi detectada uma variação acentuada na sua posição ao longo do perfil de linha, como pode ser visto na Figura 56. 


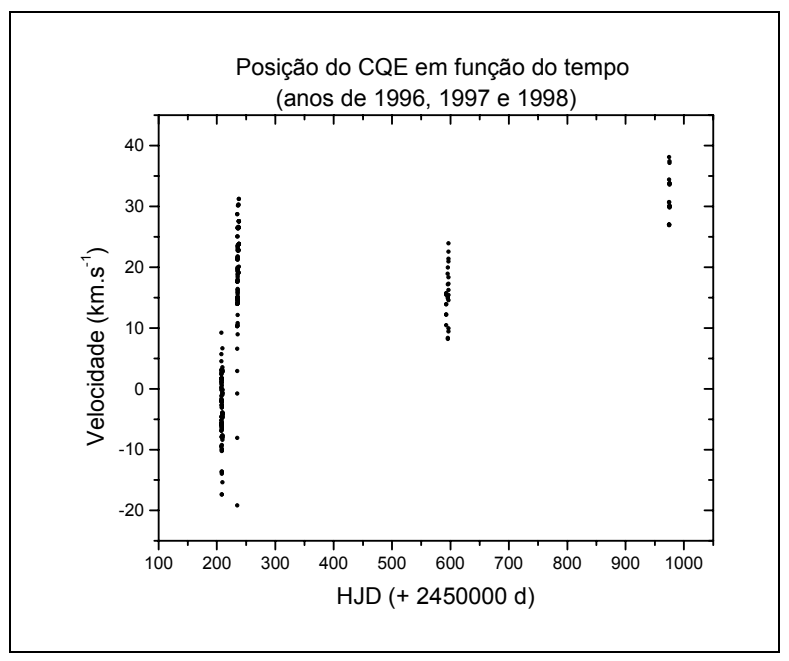

Fig.56. Gráfico da posição do $C Q E$ em velocidade ao longo do perfil de linha do He I em função do tempo.

Os $C Q E$ 's apresentaram não só variabilidade na sua posição no perfil de linha, como também variação de intensidade, desaparecendo em alguns períodos. O surgimento de um $C Q E$ pode estar relacionado a um fenômeno fotosférico (Zorec, 1988; Zorec, 1994), entretanto a variabilidade na sua intensidade pode estar ligada a um fenômeno circunstelar (Rivinius, 1999). A variabilidade na posição ao longo do perfil de linha já foi relatada em outras estrelas, como por exemplo em $\varepsilon$ Cap (Rivinius, 1999).

Aplicando-se o algoritmo CLEANEST ao conjunto de dados referente às posições dos $C Q E$ 's no perfil de linha durante 3 anos de observação (1996, 1997 e 1998), obtemos os resultados mostrados na tabela seguinte.

\begin{tabular}{ccccc}
\hline $\begin{array}{c}\text { Freqüência } \\
( \pm 0.001 \mathrm{c} / \mathrm{d})\end{array}$ & Período $(\mathrm{d})$ & $\chi^{2}$ & Graus de liberdade & Significado \\
\hline 0.017 & $\sim 60.09$ & 60 & 6 & $99.5 \%$ \\
1.144 & $\sim 0.87$ & 60 & 6 & $99.5 \%$ \\
4.673 & $\sim 0.21$ & 60 & 6 & $99.5 \%$ \\
\hline
\end{tabular}

Tab. 25. Resultados da análise temporal dos dados de posição dos $C Q E$ 's referentes aos anos de 1996, 1997 e 1998. O período de maior amplitude é o de 60 dias $(0.017$ c/d). 


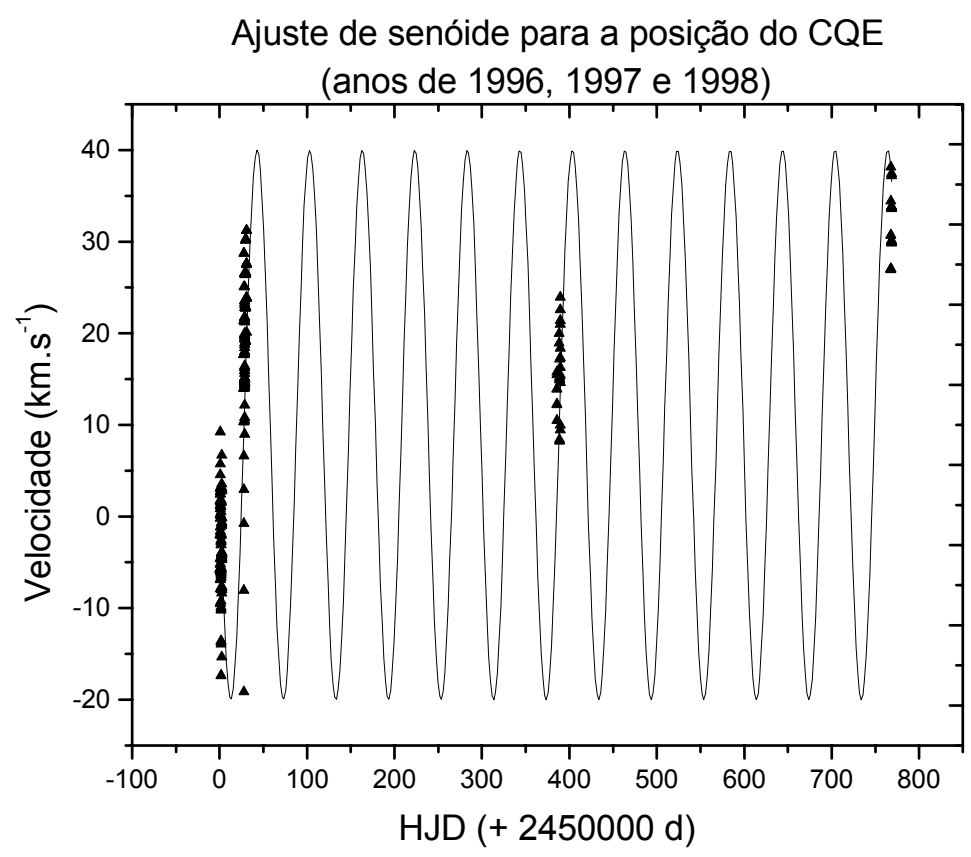

Fig.57. Ajuste de senóide com período $\mathrm{p}=60.096$ dias (linha cheia) para as posições dos $C Q E$ 's (triângulos).

O perfil médio pouco intenso de Ha observado no ano de 1996 é um indicativo da presença de um disco circunstelar de pequena extensão espacial. Nos anos de 1997 e 1998, a intensidade nessa linha aumentou significativamente.

A escolha de um modelo satisfatório para os $C Q E$ 's não é uma tarefa simples, uma vez que todos possuem considerável embasamento físico e descrevem bem a formação das emissões centrais. Os mecanismos de variabilidade rápida na posição dos $C Q E$ 's, ainda permanecem em aberto. Entretanto, é possível que a variabilidade rápida esteja relacionada com a presença de matéria na forma de blobs na envoltória da estrela. 


\section{CONCLUSÃO E PERSPECTIVAS}

Neste trabalho, foram comparados os métodos de análise temporal CLEAN e CLEANEST através de seus desempenhos na determinação de freqüências em séries temporais sintéticas. Ambos os métodos são eficazes na determinação de multifreqüências, entretanto o CLEANEST se sobressai na média dos testes tanto na determinação dos períodos quanto na determinação de suas amplitudes.

Ainda assim, estes métodos devem continuar sendo aperfeiçoados para que, no futuro, possam ser identificados sinais periódicos com amplitudes cada vez menores. Dessa forma, será possível à Sismologia estelar obter resultados importantes para outros tipos de objetos, além do Sol e das Anãs Brancas.

A análise sismológica dos dados espectroscópicos de $\eta$ Centauri, realizada neste trabalho, resultou em freqüências de PNR que concordam com os resultados obtidos por outros trabalhos sobre esta estrela (por exemplo Janot et al, 1999, Balona et al,1999). Foram identificadas as freqüências associadas a $l p v$ 's de $0.6 \mathrm{c} / \mathrm{d}, 1.48 \mathrm{c} / \mathrm{d}$, $3.8 \mathrm{c} / \mathrm{d}, 5.3 \mathrm{c} / \mathrm{d}$ (e seu primeiro harmônico) e $9.2 \mathrm{c} / \mathrm{d}$. A variabilidade multiperiódica parece estar definitivamente estabelecida para essa estrela, e podemos interpretar essa variabilidade em termos de pulsações não-radiais. De fato, a teoria de pulsações prediz a ocorrência de modos instáveis em estrelas $\mathbf{B}$, excitadas pelos mecanismos de opacidade e variabilidade da taxa de reações nucleares. A estrela apresenta variabilidades nos perfis de linha em escalas de tempo de anos, dias e horas. As variações de longo termo são relacionadas com o fenômeno de perda de massa, e as variabilidades de curto termo podem ser explicadas pela passagem de PNR's. Dentre as freqüências determinadas através das análises temporais, as de grande período e amplitude (baixas freqüências) podem ser atribuídas a modos-g, enquanto que as altas freqüências (menos intensas, períodos de $\sim$ horas) podem ser atribuídas a modos-p. Nesse contexto, a estrela $\eta$ Cen pode ser considerada uma estrela híbrida, e representada no diagrama HR na região limítrofe entre as estrelas $\beta$ Cep (pulsadores p) e as estrelas variáveis "clássicas" SPB (pulsadores g), por apresentar tanto os modos-p quanto os modos-g.

O resultado da fotometria HIPPARCOS é igual ao obtido por Balona (1999) para essa estrela e está relacionado com o período de rotação. A freqüência de rotação representa $70 \%$ da freqüência crítica e é insuficiente para assegurar sozinha a perda de massa.

Os resultados das análises da variabilidade $\mathrm{V} / \mathrm{R}$ apresentam períodos de oscilação de 12 horas a 2 dias, sendo possível atribuir essa variabilidade das asas da linha ao movimento de matéria em órbita da estrela.

A ocorrência dos CQE's é bem descrita pelo modelo de rotação diferencial (Zorec, 1988), entretanto os mecanismos de variabilidade na intensidade dos CQE's parecem estar relacionados com a passagem de matéria no disco circunstelar pela linha de visão. Os mecanismos da variabilidade na posição dos CQE's no perfil de linha ainda são obscuros.

A validade dos resultados obtidos em freqüência para as $l p v$ 's em $\eta$ Centauri é reforçada pela constante identificação das mesmas freqüências de oscilação nos conjuntos de 1996, 1997, 1998, 1997+1998, 1996+1997+1998.

A partir dos periodogramas obtidos pelo CLEANEST, é bastante clara a presença de multi-freqüências, o que reforça o modelo de Pulsações Não Radiais como explicação para a fenomenologia. 
No futuro, serão analisados conjuntos maiores de espectros de forma a se determinar as variabilidades de longo termo em $\mathrm{V} / \mathrm{R}$ e caracterizar a estrutura do envelope circunstelar. Esses conjuntos de observações foram obtidos nos últimos 10 anos e permitirão o estudo da evolução temporal das amplitudes das oscilações durante esse período, bem como o estudo de outros perfis de linha espectroscópicos.

Concluindo, devem existir vários mecanismos presentes nas estrelas de tipo Be responsáveis pelas variabilidades observadas nos perfis de linha, entretanto é certo que um deles deve ser o mecanismo de PNR. 


\section{REFERÊNCIAS BIBLIOGRÁFICAS}

- Baade,D.,1990, in: Willson L.A., Stalio R. (eds.) Angular Momentum and Mass Loss for Hot Stars, NATO ASI Series C Vol. 316, p.177

- Baade,D., Balona,L.A., 1994, Periodic variability of Be stars: nonradial pulsations or rotational modulation?, In: Pulsation Rotation and Mass Loss in Early Type Stars, Balona,L.A., Henrichs,H.F., Le Contel,J.M. (eds), IAU Symp. 162, p.311, Dordrecht, Kluwer

- Balona,L.A., 1990, MNRAS, 245, 92

- Balona,L.A., Henrichs,H.F., Le Contel,J.M. (eds), 1994,IAU Symp. 162, Pulsation Rotation and Mass Loss in Early Type Stars, Dordrecht, Kluwer

- Balona,L.A., 1995, MNRAS, 277, 1547

- Balona, L.A., Corotating photospheric clouds in the Be star $\eta$ Cen, MNRAS, 1999, 306, 407

- Bjorkman,J.E., Cassinelli,J.P., 1993, ApJ 409, 429

- Böhm-Vitense, E., Introduction to Stellar Astrophysics, vol.1, Cambridge University Press, 1989

- Christensen-Dalsgaard, J., Gough, D. e Toomre, J. 1985 Science, 229, 923

- Christensen-Dalsgaard, J., Lecture notes on stellar oscilations, Aarhus Universitet, 1989

- Cox,A.N., Morgan,S.M., Rogers,F.J., Iglesias,C.A., 1992, ApJ 393, 272

- Dachs,J., Engels,D., Kiehling,R., 1988, A\&A 194, 167

- Emílio, M., Leister, N.V., Estudo das Variações do Semi Diâmetro Solar, Dissertação de Mestrado, IAG-USP, 1997

- Feinstein, A., Marraco, H.G., 1979, AJ 84, 1713

- Foster, G. 1995, AJ 109, 1989

- Gautschy,A., Saio,H, Stellar pulsations across the HR diagram: part 1,A\&A ann.rev. 33:75-113, 1995

- Gehrz, R.D., Hackwell,J.A., and Jones,T.W. 1974, Astrophys. J., 191, 675

- Gies D.R., 1994, in IAU Symp. 162, Pulsation Rotation and Mass Loss in Early Type Stars, eds L.A.Balona and H.F.Henrichs, and J.M.le Contel

- Gies,D.R., Kullavanijaya,A.,1988, ApJ 326, 813

- Hanuschik,R.W., 1994, in L.A.Balona et al. (eds.), Pulsation, rotation and mass loss in Early-Type stars, IAU Symp. 162, p.358

- Hanuschik,R.W., Hummel,W., Dietle,O., Sutorius,E.,1995, A\&A 300, 163-176

- Hanuschik,R.W., Vrancken,M.,1995, Inf.Bull.Variable Stars 4258,1

- Hanuschik,R.W.,1995a, A\&A 295, 423

- Hanuschik, ,R.W., Hummel,W., Dietle,O., Thimm,G., 1996, A\&A Suppl. 116, 309-358

- Harmanec,P., 1988, Bull. Astron. Inst. Czechosl. 39, 329

- Harvey, J.W., Kennedy, J.R. e Leibacher, J.W. 1987, S\&T, p.470

- Hill, G.M.,Walker,G.A.M.,Yang,S.,Harmanec,P., 1989, Pub.A.S.P.,101,273

- Hiltner, W.A.,Garrison,R.F.,Schild,R.E.,1969, ApJ 157, 313

- Högbom,J.A., 1974, AAS, 15,417

- Hutchings,J.B., Nemec,J.M., Cassidy,J., 1979, PASP 91, 313 
- Janot-Pacheco,E., Jankov,S., Leister,N.V., Hubert,A.M.,Floquet,M., Multiperiodicity of the Be star $\eta$ Centauri from spectroscopic and photometric observations, 1999, $\mathrm{A} \& \mathrm{~A}, \mathbf{1 3 7 , 4 0 7}$

- Jaschek, C., Jaschek, M., Kucewicz, B., 1964, ZAp 59, 108

- Jaschek, C., Jaschek, M., 1992, A\&A 95, 535

- Jeffery,C.S., 1991, M.N.R.A.S. 249, 327

- Johnson,H.I., Mitchell,R.I., Iriarte,B., Wisniewski,W.Z., 1966, Comm.Lunar

Planet.Lab. 4, 99

- Kambe,E., et al., 1993, PASP 105, 1222-1231

- Kambe,E., Ando,H., Hirata,R.,1993a, A\&A 273, 435

- Kippenhahn,R., Weigert,A., 1994, Stellar Structure and Evolution, Springer

- Lamers,H., Pauldrach,A., 1991, A\&A 244,L5

- Leister, N.V. et al, 1994, Detection of "moving bumps" for the Be star $\eta$ Centauri, A\&A 287, 789-794

- Leister, N.V., Janot-Pacheco,E., Buck, M.C.T. et al, 1994a, in Pulsation, Rotation and Mass Loss in Early Type Stars, Balona L.A., Henrichs, H.F., LeContel, J.M. (end.), IAU Symp 162, pg. 106-107, Kluwer, Dordrecht

- Moskalik,P., Dziembowski,W.A., 1992, A\&A 256,L5

- Mourard,D., Bosc,I., Labeyrie,A., Koechlin,L., Saha,S., 1989, Nature 342, 520

- Owocki,S., Cranmer,S., Gayley,K., 1996, ApJ 472, L115

- Poeckert, R., Marlborough, J.M., 1976, Astrophys.J., 206, 182.

- Quirrenbach,A., 1994, in L.A.Balona et al. (eds.), Pulsation, rotation and mass loss in Early-Type stars, IAU Symp. 162, p.450

- Quirrenbach,A., Bjorkman,K.S., Bjorkman,J.E., et al. 1997, ApJ 479, 477

- Rivinius,Th., Nature and activity of the Be star $\mu$ Centauri, Tese de Doutoramento, Ruprecht-Karls-Universität Heidelberg, 1998

- Rivinius,Th., Štefl,S., Baade,D., Central quasi-emission peaks in shell spectra and the rotation of disks of Be stars, 1999 A\&A 348,831

- Roberts, D.H., Lehar, J. \& Dreher, J.W. 1987, AJ 93, 968

- Slettebak, A., Snow,T.P.(eds),1987, Physics of Be stars, IAU Coll.№ 92, Cambridge, Cambridge Univ.Press

- Slettebak, A. 1988, PASP, 100, 770

- Smith, M.A. 1977, ApJ 215, 574

- Smith,M.A., 1989, ApJS 71, 357

- Smith, M.A., Cohen,D.H., Hubeny,I. et al., 1997, ApJ 481, 467

- Stee,P., de Araújo,F.X., Vakili,F. et al., 1995, A\&A 300, 219

- Štefl,S., Baade,D., Harmanec,P., Balona,L.A., Simultaneous photometric and spectroscopic monitoring of rapid variations of the Be star $\eta$ Cen, 1994, A\&A (preprint)

- Štefl,S., Hummel,W., Rivinius, Th.., The circumstellar structure of the Be shell star $\phi$ Per, 2000, A\&A (preprint)

- Telting,J.H., Schrijvers,C. 1997a, Line-profile variations due to adiabatic non-radial pulsations in rotating stars, paper I, A\&A 121, 343-368

- Telting,J.H., Schrijvers,C. 1997b, Line-profile variations due to adiabatic non-radial pulsations in rotating stars, paper I, A\&A 317, 723-741 
- Telting,J.H., Schrijvers,C. 1997c, Line-profile variations due to adiabatic non-radial pulsations in rotating stars, paper I, A\&A 317, 742-748

- Townsend R.H.D., 1997, MNRAS 284, 839

- Underhill, A., Doazan, V.(eds.), B Stars with and without emission lines, 1982, NASA/CNRS SP- 456 monograph

- Unno, W., Osaki, Y., Ando, H. \& Shibahashi, H. 1989, Nonradial Oscillations of Stars, 2nd ed. (Tokyo: Univ.of Tokyo Press)

- Vakili,F., Mourard,D., Stee,P., 1994, in L.A.Balona et al. (eds.), Pulsation, rotation and mass loss in Early-Type stars, IAU Symp. 162, p.435

- Vogt,S.S., Penrod,G.D.,1983, ApJ 275, 661

- Walker, G.A.H., Yang, S. e Fahlman G.G. 1979, ApJ, 233, 199

- Yang,S., Ninkov,Z., Walker,G.A.H., 1988, A.S.P., 100, 233

- Zorec, J., Mochkovitch,R., Garcia,A., 1988, C.R.Acad.Sci.Paris, t.306, Série II, p.12251230

- Zorec, J., 1994, in: Balona L.A., et al. (eds) Pulsation, Rotation and Mass Loss in Early Type Stars, IAU Symp. 162, p.257 